\title{
DEVELOPMENT OF NEUTRON LEAKAGE SPECTROMETERS WITH EMPHASIS ON THE kEV RANGE
}

\author{
Rudolph J. Henninger
}

(Ph. D. Thesis)

\section{March 1971}

AEC Contract No. W-7405-eng -48

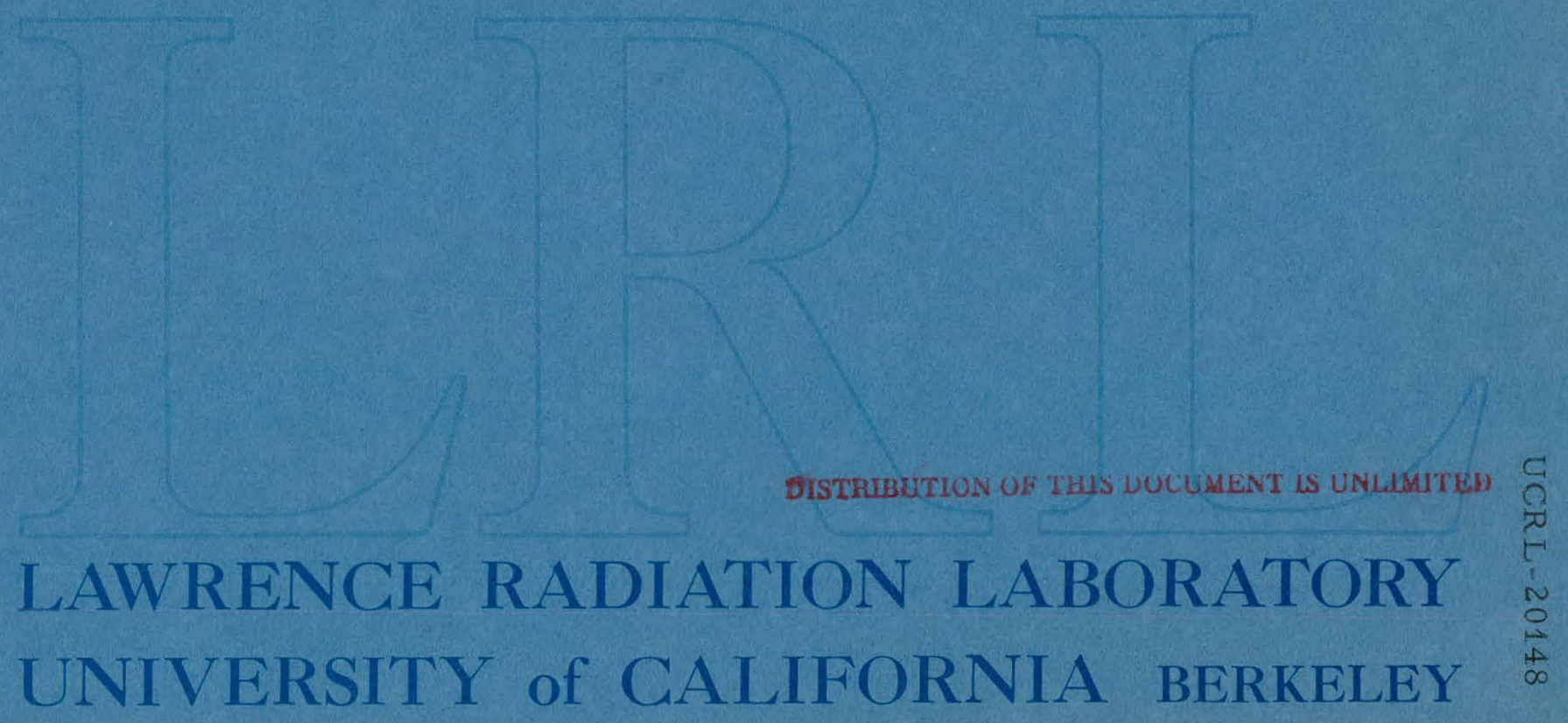




\section{DISCLAIMER}

This report was prepared as an account of work sponsored by an agency of the United States Government. Neither the United States Government nor any agency Thereof, nor any of their employees, makes any warranty, express or implied, or assumes any legal liability or responsibility for the accuracy, completeness, or usefulness of any information, apparatus, product, or process disclosed, or represents that its use would not infringe privately owned rights. Reference herein to any specific commercial product, process, or service by trade name, trademark, manufacturer, or otherwise does not necessarily constitute or imply its endorsement, recommendation, or favoring by the United States Government or any agency thereof. The views and opinions of authors expressed herein do not necessarily state or reflect those of the United States Government or any agency thereof. 


\section{DISCLAIMER}

Portions of this document may be illegible in electronic image products. Images are produced from the best available original document. 


\section{PAGES $\mathrm{i}$ to $\mathrm{ii}$ WERE INTENTIONALLY LEFT BLANK}


TABLE OF CONTENTS

Page

I. INTRODUCTION

II. THEORY

A. Interpretation of Measurements by a

Leakage spectrometer

B. Determining the spectrum from the

Measurements

C. Method of Calculating Response Functions

D. Description of Input. Data and Test

Calculations

E. Results

III. EXPERIMENT

A. Apparatus and Experimental Results

B. Analysis of Experiment and Comparison

of Results

IV. SUMMARY OF RESULTS AND CONCLUSIONS

APPENDICES

Appendix A. Development of Group Equations Used in ANISN

Appendix B. Tests of ANISN

Appendix C. Cross Sections and Energy. Transfer Array

REFERENCES

\section{LEGAL NOTICE}

This report was prepared as an account of work sponsored by the United States Government. Neither the United states nor the United States Atomic Energy Commission, nor any of their employees, nor any of Commission, nor any of their employees, nor any of makes any warranty, express or implied, or assumes any legal liability or responsibility for the accuracy, completeness or usefulness of any information, spparatus, product or process disclosed, or represents that its use would not infringe privately owned rights. 
$-i v-$

\section{DEVELOPMENT OF NEUTRON LEAKAGE SPECTROMETERS \\ WITH EMPHASIS ON THE keV RANGE}

Ph.D.

Rudolph:J. Henninger

Nuclear Engineering

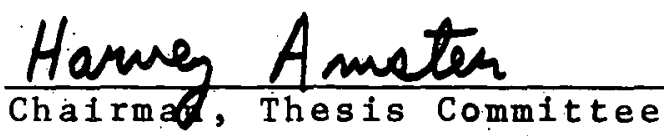

\section{ABSTRACT}

Calculations were performed to design and analyze a set of neutron spectrometers consisting of cadmium-covered, spherically-shaped, resonance-absorbing cores, surrounded by various-sized polyethylene spheres. Some of the detectors had an additional layer of boron around the polyethylene. Because of the combined effects of leakage (hence the name) and moderation in the polyethylene, the measured activation of the resonance absorber depends on the incident neutron energy; with a maximum in an energy range determined by the sizes and properties of all the materials. This response is proportional to the adjolnt flux, which is calculated by the multigroup discrete ordinate transport code ANISN. The adjoint boundary condition is the probability that a neutron entering the core is captured there. It is assumed that this probability is independent of the presence of the surrounding materials.

Calculations were performed for polyethylene sphere d. ameter varying from 2 In. to 10 1n. to find a set useful in the keV region. Some of these calculations were compared 
with experiments performed by S. Binney and Hans Mark using nearly monoenergetic keV photorieutrons: The experimental results were typically about $30 \%$ different from those calculated. 


\section{INTRODUCTION}

The measurement of neutron energy distributions. is Important for many applfcations of nuclear energy. Checking calculations that predict the behavior of the "fast" reactors now belag developed require this knowledge. This need is particularly acute in the energy region between $1 \mathrm{keV}$ and $1 \mathrm{MeV}$ since most of the fission events are inftiated there. For any source of neutrons, calculation of both the neutron induced blological dose rate and flux attenuation by shlelding material require knowledge of the spectrum, since the relative biological effectiveness depends strongly on neutron energy.

Wtthin the thermal and epithermal ranges, foll activation techniques have been employed extensively to measure spectra. This method is very convenient since the folis are very small and can easily be placed in many positions instde of assembiles. They are effictent, so that very small quantities of material can be used, hence the flux distribution 1s not disturbed. The cross sections for activation in this energy range are well known, and, since it 18 the radioactivity induced in the foil that is measured, the readings do not suffer from interference by gama rays nor "flooding" from intense neutron pulses. Finally, the existence of large resonances in the cross sections at epithermal energles makes it relatively easy to interpret the activation measurements in terms of the neutron flux 
over a narrow energy range.

Activation methods, however, do not work well in the energy region between $1 \mathrm{keV}$ and $1 \mathrm{MeV}$. At these energies, there are no lsolated activation resonances which can be used to define a narrow band; also, the cross sections have not been measured precisely enough. Mechanical choppers have also been used with success in the eV range but above the low keV range their resolution becomes unacceptable.

Above 1 MeV opectra can be measured by means of threshold detectors, nuclear emulsions, semiconductor detectors or protion recoll spectrometers. Attempts to exténd these methods downward. In energy have met with gerious diffculties. Thresholds for nuclear reactions are too high (above $1 \mathrm{MeV}$ ) for this method to be used. Proton recol1, nuclear emilsion, and semiconductor detectors will operate in the keV range, but generally resolution restricts their usefulness to the very high keV range and above (a good review of the applicability of these detectors can be found In Ref. K160). Good measurements in the keV range can be obtained with time-of-flight methods, but complicated equipment is required as well as an aperture for bringing out the neutron beam.

In consideration of this gap in the field of neutron spectrometry, the purpose of this work will be to adapt activation techniques, with thelr inherent advantages, 
to measure neutron energy distributions in the keV range. Gold foil activation hag been a traditional method for determining certain flux properties in thermal reactors. The reaction

$$
197 \mathrm{Au}(\mathrm{n}, \gamma){ }^{198 \mathrm{Au}}
$$

has a strong resonance at an incident neutron energy of 4.9 eV. Below this energy, the cross section is approxImately inversely proportional to the square root of the incident neutron energy (see Figure 1 ).

A limited amount of spectral information can be obtained by the so-called cadmium ratio measurements. The activation of gold folls shielded with cadmium, to remove thermal neutrons (see Figure 1), determines essentially the neutron flux near five electron volts, provided the spectrum rapidly decreases with increasing neutron energy. The difference between the counting rate of an activated bare toll and a cadmlum covered foll is a measure of the flux below the cadmium "cut-off" energy at $0.4 \mathrm{eV}$.

The same principle can be applied with other elements having strong capture resonances in their cross sections. The induced activity of a foil exposed to a neutron flux $\phi(E)$ is proportional to the integral

$$
I=\int \phi(E) \sigma(E) \mathrm{dE}
$$




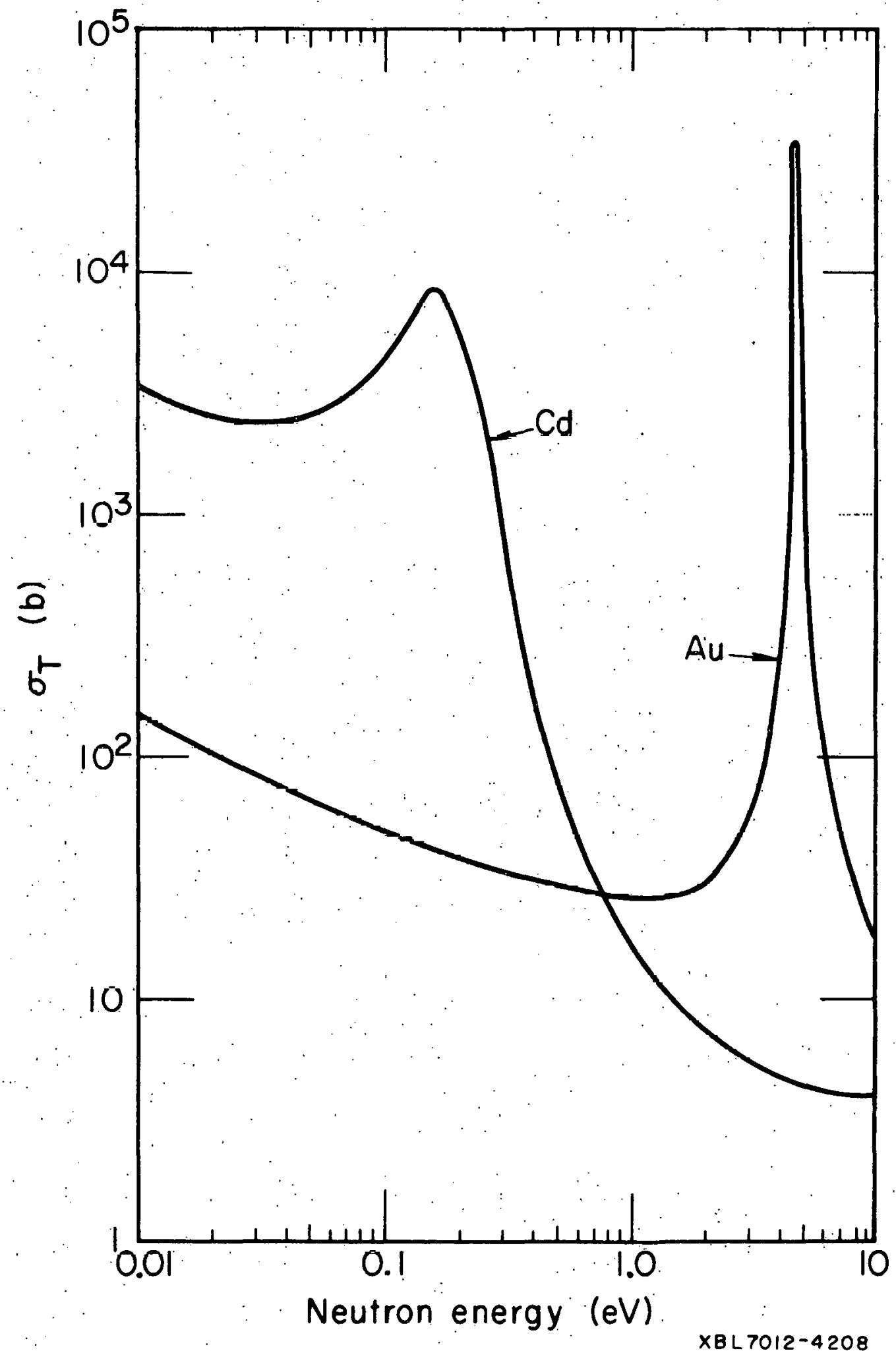

FIGURE 1. Total Cross Sections for Gold and Cadmium 
If the flux has the form

$$
\phi \backsim \frac{1}{E} \text {, }
$$

which is approximately the case for the epithermal flux in a large non-absorbing system, and the cross section $\sigma(E)$ is dominated by a single large resonance, then the activation of a cadmium covered foil is given by the resonance integral

$$
I_{\text {res }}=\int_{.4 \mathrm{eV}}^{\infty} \sigma(E) \frac{\mathrm{dE}}{\mathrm{E}}
$$

where the lower limit has been set at the cadmium cut-off energy. The cadmium cover 1 s used to eliminate the large activation due to thermal neutrons. Table 1 lists the elements generally used as resonance absorbers, along with the half-ilfe of the 1sotopes resulting from capture, and the resonance integral.

Several theoretical methods for converting the observed activities of the different isotopes into usable Information about the neutron spectrum have been devised for the region indicated in Table 1 . However, the activation method described thus far are not useful in the energy region of interest without further modification.

A "spectrum shifting" method will now be described which will make $1 \mathrm{t}$ possible to use these methods at higher 
TABLE 1

ISOTOPES USED FOR ACTIVATION ANALYSIS

\begin{tabular}{|c|c|c|c|}
\hline Element & $\begin{array}{l}\text { Resonance } \\
\text { Energy }(\mathrm{eV})\end{array}$ & $I_{\text {res }}(b)$ & $T_{1 / 2}$ \\
\hline In & 1.45 & 2060 & $54 \mathrm{~m} 1 \mathrm{n}$ \\
\hline $\mathrm{Au}$ & 4.9 & 1543 & $2.7 \mathrm{~d}$ \\
\hline $\mathrm{Mn}$ & .337 & 15 & $2.56 \mathrm{~h}$ \\
\hline W. & 18.8 & 500 & $.24 \mathrm{~h}$ \\
\hline $\mathrm{Co}$ & 132 & 65 & $.5 .2 y$ \\
\hline $\mathrm{Na}$ & 2.850 & .30 & $1.5 \mathrm{~h}$ \\
\hline
\end{tabular}


energ1es. In 1960, T.W. Bonner devised a mult1sphere technfque for measuring the neutron spectra produced by 1sotopes undergoing spontaneous fission. Br60,Bo61 This method makes use of the inherent advantages of spherical symmetry, which were originaliy pointed out by Thompson. Th53 The "Bonner" sphere consists of a lithium lodide scintillation crystal, which is sensitive to thermal neutrons, surrounded by a polyethylene sphere (Figure.2). The polyethylene $\left(\mathrm{CH}_{2}\right)$ acts as a moderator for incident neutrons, slowing them to thermal energies, where they can be detected by the lithium lodide crystal.

The response of each sphere was measured by means of monoenergetic neutrons produced by a Van de Graaff acce1erator and a ${ }^{7} L(p, n)^{7}$ Be reaction. The results obtalned for different sized moderating spheres is shown in Figure 3. As can be seen in the figure, the response functions peak in the energy range from about .3 eV to $4 \mathrm{MeV}$. The explanation of the peaking is simple: at high incldent energies, most of the neutrons leak out of the detector before they can scatter enough times to become thermallied and hence detected. On the other hand, a large fraction of the incident low energy neutrons becomes thermal before reaching the center, and therefore leaks out before being captured there. The large thermal scattering cross section of hydrogen in the polyethylene enhances this effect. A neutron entering near the peak 


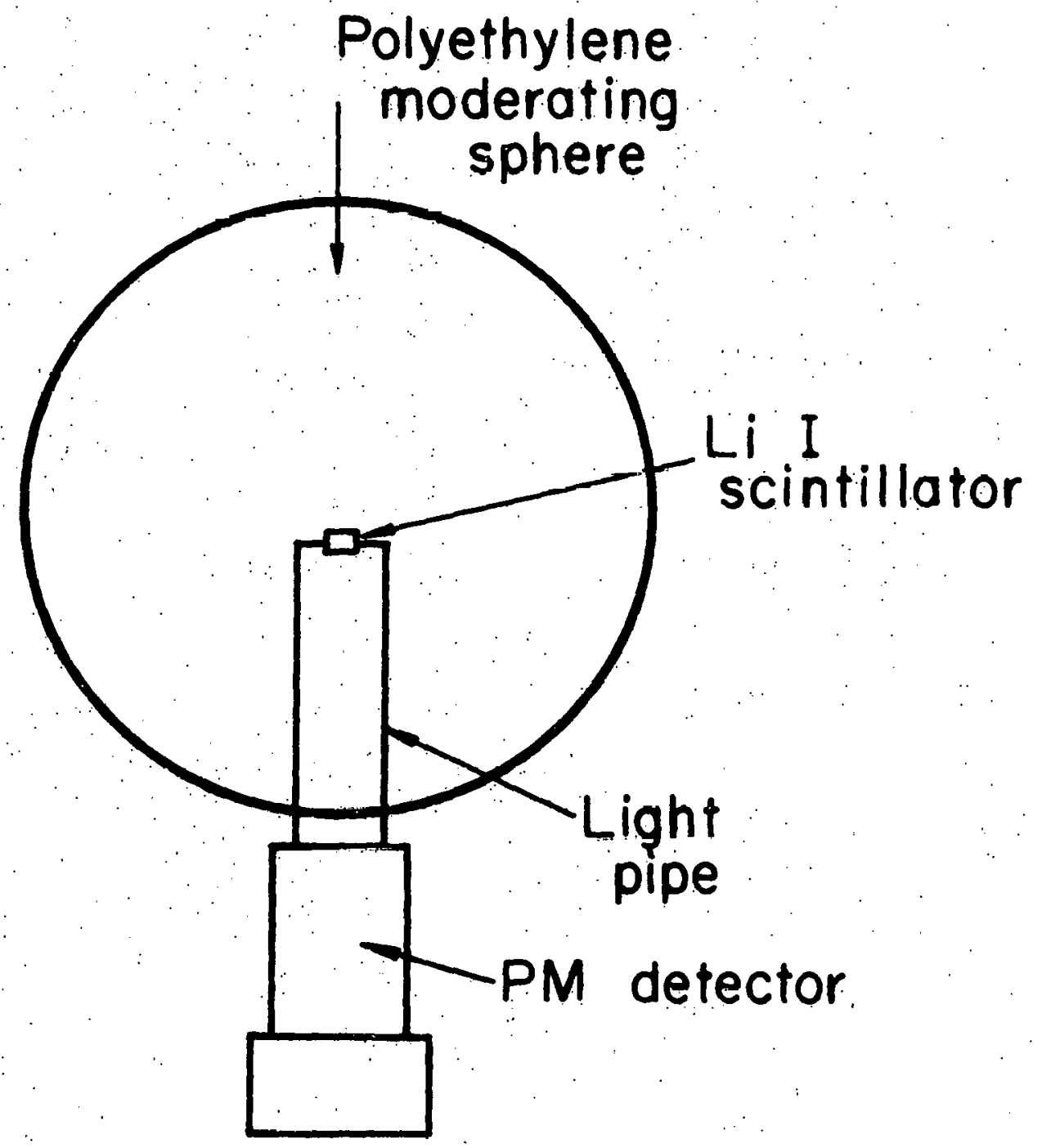

XBL7012-4206

FIGURE 2. Bonner Leakage spectrometer 


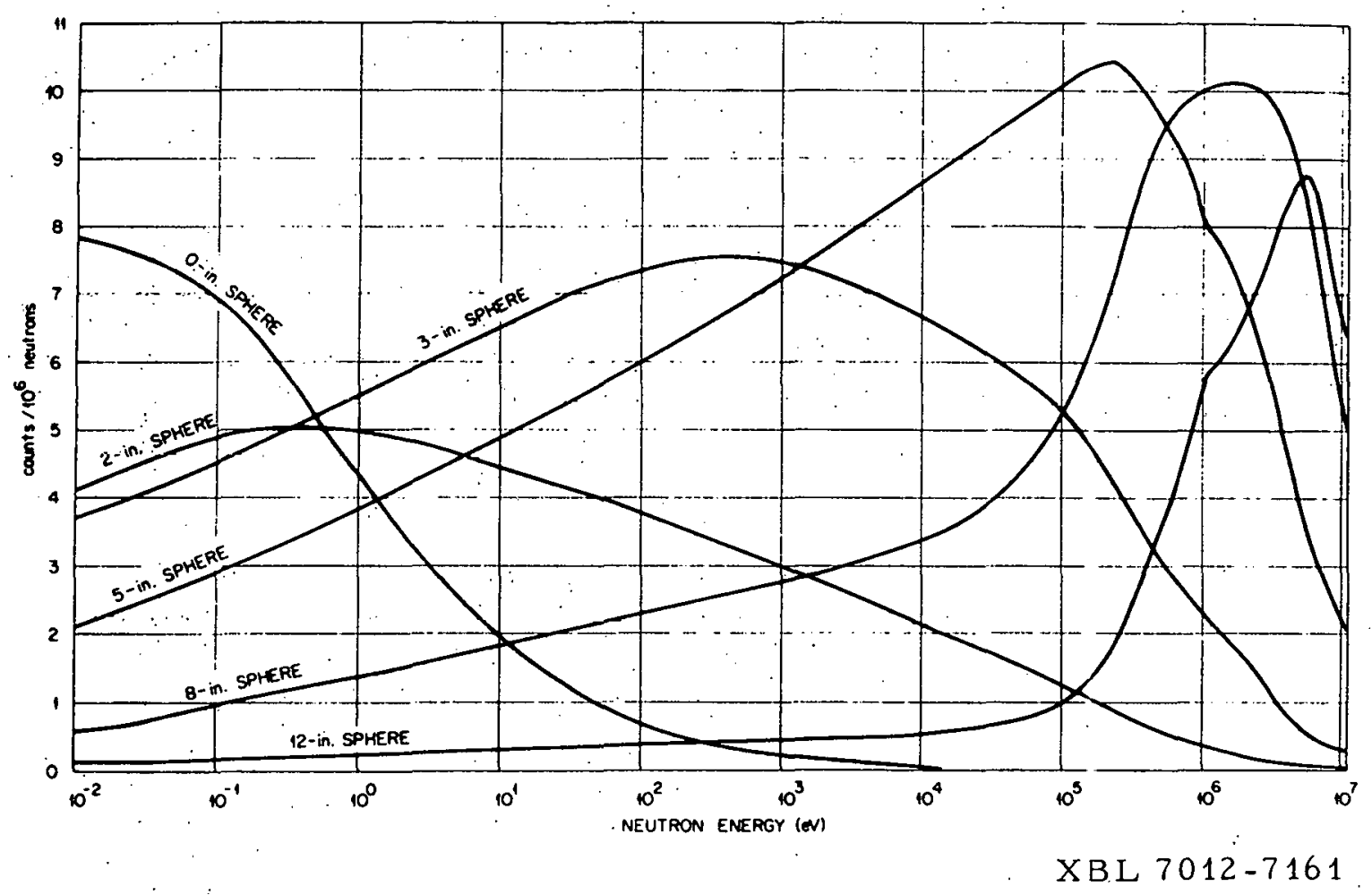

FIGURE 3. Response Functions for Bonner Spheres. Me65 
undergoes, on the average, the number of collisions required to reach thermal energy at the center. Since the peaking is caused by leakage, this type of spectrometer will be referred to here as a "leakage" spectrometer (IS).

A reduction in the size of the Bonner Leakage Spectrometer, some of which reach $12^{\prime \prime}$ In diameter, would be an Important improvement... This can be accomplished by replacing the 11 thfum lodide crystal and the necessary Ifght pipe aud photomultiplifer asoembly by somethiug that would detect neutrons at a higher energy, hence, requiring less moderator. A cadmium covered activation foll can be usèd as such a detector and, therefore, seems a good choice. In this way, Incldent high energy. neutrons (kev and above) will be shtfted to an energy regtion in whioh they can be detected more easily.

Another important advantage of using a cadmium covered resonance absorber occurs in the calculation of the response curves. Because of the scarcity of monoenergetic neutron sources in the keV range for use in callbration, calculations must be heavily reiled upon and any simplification of them is helpful. At energies above the cadmium cut-off the nuclei with which the neutrons interact may be considered stationary compared to the neutrons, and chemical binding effecto that are important mafnly at thermal energies may be neglected.

When the thermal detection is replaced by one for the 
eV range, and the amount of polyethylene is reduced so that the peak sensitivity is at the same energy, the resulting response shape remains approximately the same at all energies. In particular, the resolution of the smaller sized detectors remalns poor. The response can be considerably sharpened, however, by adding a layer of boron around the outside of the moderator. Since boron is a $1 / v$ absorber, lower energy neutrons are preferentially removed: This effect can be seen in some curves calculated by. S.K. Metha Me64, Me65 who used spheres, as Bonner had constructed, modified in this manner. Figure 4 shows the lowering of the response at low energies and the shifting of the peak response to higher energies with the addition of a layer of boron.

The proposed spectrometers consist of a cadmium covered resonance absorber surrounded by various sized polyethylene spheres (Figure 5). Some have an additional layer of boron. In the following sections, the use and design of this new spectrometer will be described, and calculated response functions will be given for several stzes and resonant absorbers.. An experiment is briefly described, the results of which are compared with the calculations. 


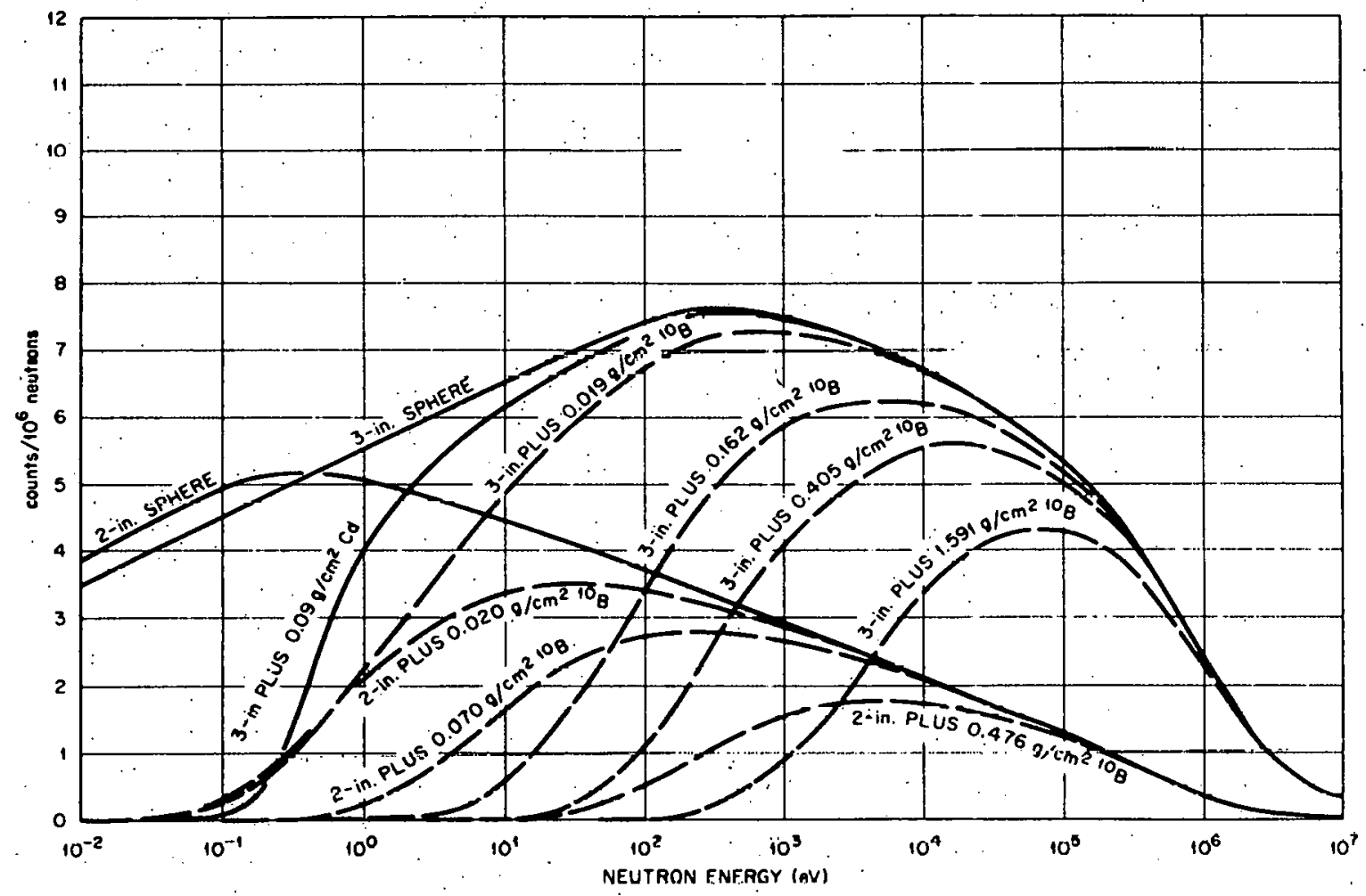

XBL $7012-7162$

\section{FIGURE 4. Response Functions for Modified Bonner spheits. Me 65}




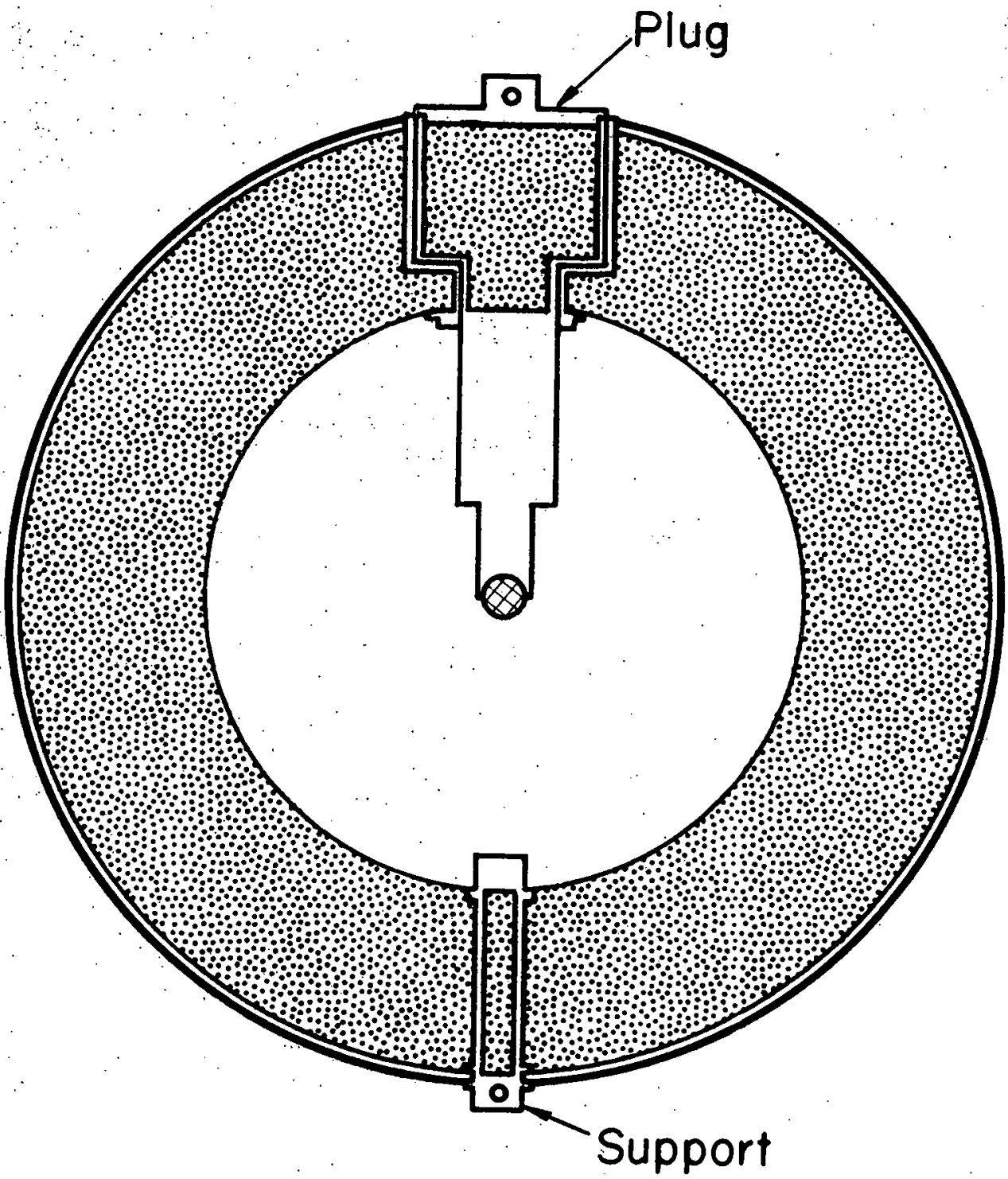

\section{Boron carbide powder In-Cd foil Polyethylene}

FIGURE 5. Activation Leakage spectrometer (cross sectional view) 


\section{THEORY}

A. Interpretation of Measurements by a Leakage Spectrometer

In this section a definition of what a Leakage

spectrometer (LS) measures will be made. Where and how

a set of LS's can be used for determining neutron spectra

w11l then be discussed.

Let $\mathrm{F}^{+}(\mathrm{E}, \mu)$ be the probability that a neutron having an energy $E$ and making an angle $\cos ^{-1} \mu$ with the outward polnting normal at the surface of the spectrometer, will be absorbed in the core material. This quantity depends on no angles other than $\cos ^{-1} \mu$ because of the spherical symmetry. Furthermore, since only incoming neutrons can be absorbed,

$$
F^{+}(E, \mu)=0 \text { for } \mu>0
$$

Let $F\left(R, \bar{\Omega}_{0}, E ; \bar{\Omega}\right)$ be the angular flux at the position

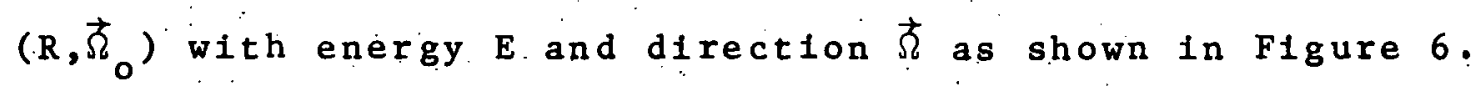
Here $R$ is the radius of the spectrometer, so the flux is evaluated at its surface. With this angular flux,

$$
F\left(R, \bar{\Omega}_{0}, E, \bar{\Omega}\right) R^{2}\left(-\vec{\Omega} \cdot \vec{\Omega}_{0}\right) \mathrm{d} \overrightarrow{\widehat{\Omega}}{ }_{0} \mathrm{~d} \vec{\Omega} \mathrm{d} E \text { neutrons/sec }
$$

enter an area $\mathrm{R}^{2} \mathrm{~d} \vec{\Omega}_{0}$ on the surface with directions in $\mathrm{d} \vec{\Omega}$ about $\vec{\Omega}$. Thus $A$, the number; of absorptions per second in the core material for the flux glven, is 


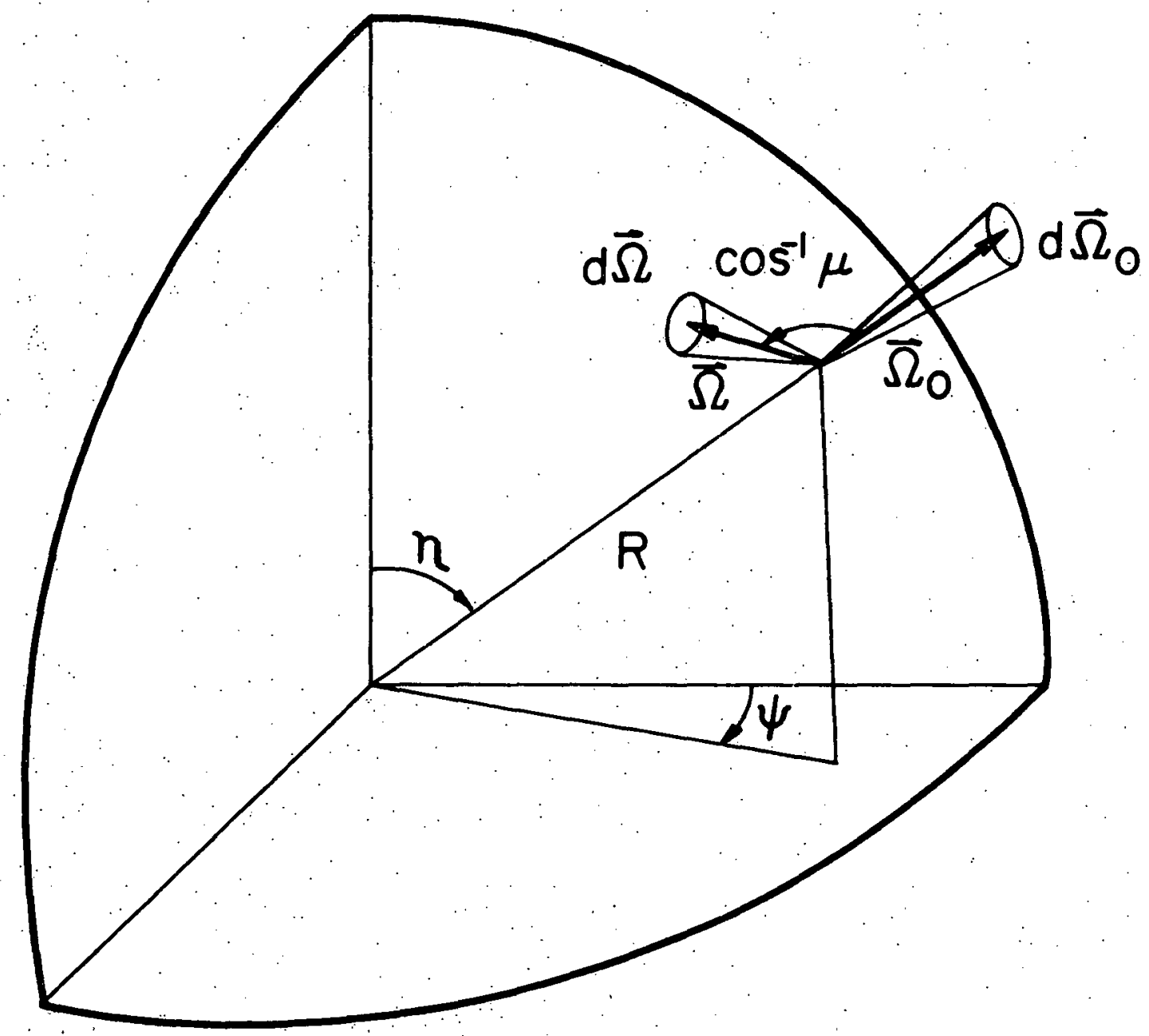

XBL7II- 27.35

FIGURE 6. Geometry for Calculating L.S. Capture Probability 
$A=R^{2} \int_{4 \pi} \mathrm{d} \vec{\Omega} 0 \int_{\vec{\Omega}} \mathrm{d} \vec{\Omega} \vec{\Omega}_{0}<0 \quad \mathrm{dEF}^{+}\left(\mathrm{E},-\vec{\Omega} \cdot \vec{\Omega}_{\mathrm{o}}\right) \mathrm{F}\left(\mathrm{R}, \vec{\Omega}_{\mathrm{o}} \mathrm{E}, \vec{\Omega}\right)\left(-\vec{\Omega} \cdot \vec{\Omega}_{\mathrm{o}}\right)$

At saturation the number of absorptions per second is equal to the activity of the core material:

We now define $\sigma_{L S}(E)$ the "effective cross section of the spectrometer",

$$
\sigma_{L S}(E) \equiv A(E) / \bar{\phi}(E)
$$

where $A(E) d E$ is the number of neutrons absorbed that enter at energies in $\mathrm{dE}$ and.

$$
\bar{\phi} \equiv \frac{1}{4 \pi} \int_{4 \pi} \mathrm{d} \vec{\Omega}_{0} \cdot 2 \int_{\vec{\Omega} \cdot \vec{\Omega}_{0}<0} \mathrm{~d} \vec{\Omega} \mathrm{F}\left(\mathrm{R}, \vec{\Omega} \mathrm{O}_{0}, \mathrm{E}, \vec{\Omega}\right)
$$

$\bar{\phi}$ will be thought of as the average inward scalar flux which under certain conditions mentioned below is the scalar flux "at" the surface of the LS. The saturated activity then becomes

$$
\Lambda=\int A(E) d E=\int \bar{\psi}(E) \cup_{L S}(E) d E
$$

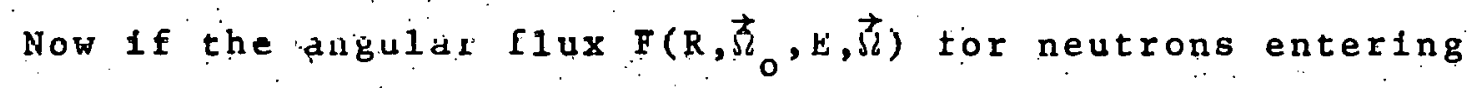
the LS 1s independent of position on the surface of the LS, then 


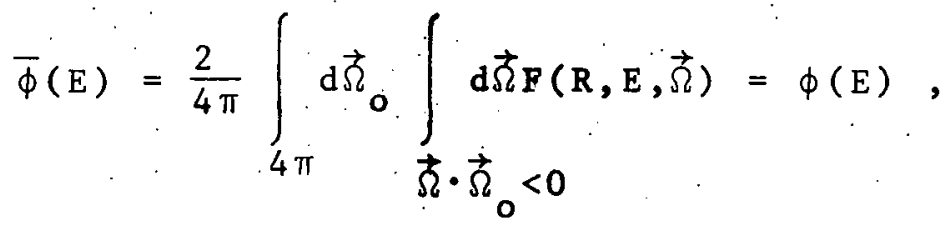

the scalar flux on the surface because the integral over $\vec{\Omega}$ is the Inward half of the scalar flux at $\vec{\Omega}_{0}$, but this Inward half covers all directions as the integral over $\vec{\Omega}_{0}$ is performed. Under this condition

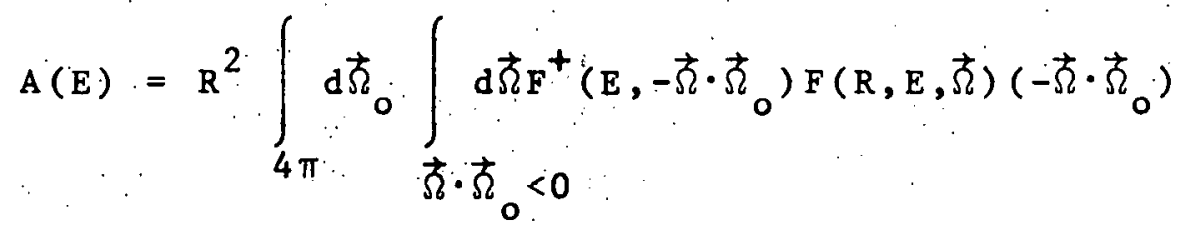

but

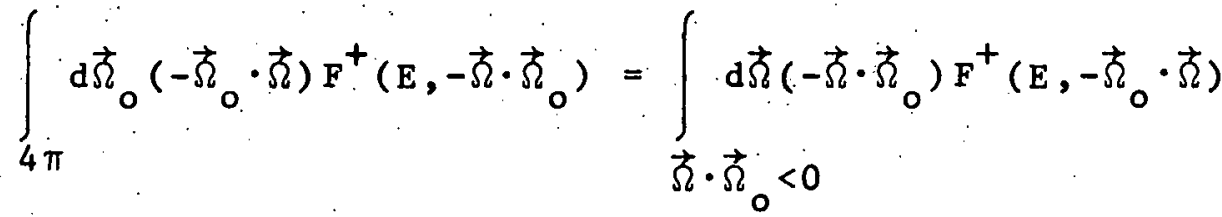

$$
\begin{aligned}
& =-2 \pi \int_{-1}^{0} \mathrm{~d} \mu \mu \mathrm{F}^{+}(\dot{\mathrm{E}}, \mu) \equiv 2 \pi \mathrm{J}^{+}(\mathrm{E})
\end{aligned}
$$

Independent of $\bar{\Omega}_{0}$ and $\vec{\Omega}$. It will be seen in a section that follows that $\mathrm{J}^{+}$is the adjoint current. Thus,

$$
A(E)=2 \pi R^{2} J^{+}(E) \int_{4 \pi} \mathrm{d} \vec{\Omega} F(R, E, \vec{\Omega})=2 \pi R^{2} J^{+}(E) \phi(E)
$$

and by definition

$$
\sigma_{L S}(E)=2 \pi \dot{R}^{2} J^{+}(E)
$$


We see then that if the incoming angular flux (no matter how anfsotroplc) is independent of position on the surface of the LS, A depends only on $\phi$, therefore scalar flux is measured directly.

For illustration, consider the special case of a black sphere $\left(F^{+}(\mu)=1, \dot{1}\right.$ e. every neutron entering is absorbed) and a monoenergetic lncident flux. For this example

$$
\sigma_{L S}=-2 \pi R^{2} \int_{-1}^{0} \mu d \mu=\pi R^{2}
$$

leading to

$$
A=\pi R^{2} \phi
$$

This equation io clearly true for a monodirectional beam, of flux $\phi$, for the black sphere will simply remove neutrons presented to its cross-sectional area: In the case of the black sphere immersed in an finfinite isotropic bath of neutrons the number absorbed ts the number crosalng per unit area (the inward current $\mathrm{J}^{-}=\phi / 4$ ) multiplied by the surface area $4 \pi R^{2}$.

If the angular flux incident on the LS varies slightiy, then the average inward scalar flux $\bar{\phi}$ is approximately equal to the scalar flux that would be "at" the center of the sphere if all neutrons actually heading for the center from the surface reached 1t. The reason is that each point on the surface contributes only one direction to the flux 
at the center, and together all the surface points give contributions from all possible directions. Besides, $\mathrm{F}^{+}(\mathrm{E}, \mu)$ is peaked at $\mu=1$ (see Figure 21), so that neutrons heading toward the center are heavily weighted in this measurement. Strong motivations for making the spectrometer as smail as possible are that $\bar{\phi}$ becomes the scalar flux and that its presence does not alter the spectrum it is to measure.

We have considered only what the LS measures in terms of the flux at the surface in the presence of the sphere. In some cases this flux may not be different from that which is present when the sphere is removed, such as if the spectrometer were to be placed in free space far from other materials with which neutrons could interact. The spectrometer would thus be useful for measuring stray neutron fields outside of shields, crittcal assemblies, or In relatively large cavities within some systems. In some systems the spectrometer may. be placed within a medium such as water and not distort the incident spectrum significantly. Thus it may be used. within aqueous shields, without having to correct the spectrum obtained.

There are situations, however, in which the incident flux will be changed by the presence of the LS. In these instances, it might be possible to correct for the influence of the LS fairly eas1ly, e.g. by considering the media as locally infinite (1 fke the treatment of thermal flux 
depression by foils) or if the geometry. is spherically symmetric (such as including in test calculations the. effects of placing an LS at the center of a large critical sphere $e^{s+68}$ ).

\section{B. Determining the Spectrum from the Measurements}

The results of the exposure of a set of $M$ spectrometers is given by $M$ integral equations of the form

$$
A_{m}=K \int \phi(E) \sigma_{L S}^{m}(E) d E \quad \quad m=1,2, \ldots, M
$$

where $k$ 1s a constant determined by the foll counting technique employed, and $\phi(E)$ is the flux for which the energy dependence is desired. One method of obtaining an appropriate expresston for the flux is to parameterize an assumed function for it and then determine the parameters. This was done by using Bonner Leakage Spectrometers to obtain an expression for fission spectra of the form $(\sqrt{E}$ exp-aE). This form was substituted into II.6, which was then evaluated numerically to obtain $\alpha$. Bonner performed measurements with spheres having different radii to verify the procedure. If the same $\alpha$ wa obtained for each sphere, the method was considered valid. More general types of spectra would require more parameters. One method might be a multigroup analysis; in which the neutron flux is expressed as a sum of $N$ group fluxes $\phi\left(E_{n}\right)$. $A_{m}$, the activity of the core for a particular spectrometer m, is 
assumed to be given by

$$
A_{m}=k \sum_{n=1}^{N} \phi\left(E_{n}\right) \int_{\Delta E_{n}} \sigma_{L S}^{m}(E) d E \quad m=1,2, \ldots, M
$$

or

$$
\vec{A}=\kappa \underline{\underline{\Phi}}
$$

where $\sum$ is an $M x N$ matrix and $\vec{A}$ and $\vec{\phi}$ are column vectors. If this experiment is performed for $M(=N)$ different radius-foil combinations, the matrix equation II. 8 becomes a set of simultaneous equations. The group fluxes can be solved for if the responses are linearly independent. A generalized form of II.8 can be obtained by assuming that the neutron spectrum is expressed by a sum of $N$ iinearly independent given functions $f_{n}(E)$,

$$
\phi(E)=\sum_{n=1}^{N} a_{n} f_{n}(E)
$$

Since the number of detectors equals the number of activity. equations, the $N$ parameters $a_{n}$ can be determined uniquely only if $N$ is not greater than the number of detectors.

Equations like II.6 for bare foil activation have been solved by using iterative techniques. Mc67, Mc69 This procedure involves selection of an Initial spectral approximation and its subsequent correction to obtain 
a fit to the activation data. A least squares method has alsobeen used with success to determine the coefflcients $a_{n}$ of II.9 by minimizing the quadratic form

$$
Q=\sum_{m=1}^{M}\left[\frac{A_{m}-k \cdot \sum_{n=1}^{N} a_{n} \int_{0}^{\infty} f_{n}(E) \sigma^{m}(E) d E}{A_{m}}\right]^{2}
$$

Di 65

Go1d ${ }^{\text {Go64 }}$ has stated that, in general, the approximate. solitinn ghould proporiy oatisfy lie sul of Incegrai equations II.6 and certain subsidiary conditions imposed by physical considerations (non-negativity, etc). A generalized formalism, which includes a controlled degree of smoothness or closeness to a given approxtmate solution for II.6, was introduced and coded hy Routt 1. Ro69

Calculations of responses for a set of spectrometers which lend themselves to the above methods for spectrum determination wi11 now be described.

\section{Method of Calculating Response Functions}

This section will be devoted to the methods for determining $\sigma_{L S}(E)$; the function which, if multiplied by the "scalar" flux and integrated over energy, yieldy the saturated activity of the absorbing core of the LS. The connection between $F^{+}(E, \mu)$ from which $\sigma_{L S}(E)$ is generated, and the adjoint flux will be made. It will be seen how the adjoint flux is determined by the code ANISN ${ }^{\text {En } 67}$. using a boundary condition and/or adjoint source. 
The adjoint flux may be interpreted as follows. Let $\underline{H}$, called the transport operator, be defined as

$$
\begin{aligned}
\underline{\mathrm{H}} F & \equiv \vec{\Omega} \cdot \nabla F+\sum_{t} F-\int \mathrm{d} \mathrm{u}^{\prime} \int \mathrm{d}^{\mathrm{\Omega}} \cdot \mathrm{F}(\overrightarrow{\mathrm{r}}, \mathrm{u}, \vec{\Omega}) \Sigma_{\mathrm{S}}\left(\mathrm{u}^{\prime}+\mathrm{u} ; \vec{\Omega} \cdot \cdot \vec{\Omega}\right) \\
& =\mathrm{S}(\overrightarrow{\mathrm{r}}, \mathrm{u}, \vec{\Omega})
\end{aligned}
$$

where $F(\vec{r}, u, \vec{\Omega})$ is the angular flux as In. II.l with the change of variable $u=\ell n . E_{0} / E$ ( $u$ is called the neutron lethargy); $E_{0}$ is some reference energy (here $E_{0}=10^{7}$ eV). The first and third terms on the right hand side represent neutron gains to the phase space point $\vec{p}=(\vec{r}, u, \vec{\Omega})$ from streaming and scattering from higher energles respectively, and the second term represents removal from $\vec{p}$ due to collisions. I's are macroscopic cross sections, and $s(\vec{r}, u, \vec{\Omega})$ is an external source at $\vec{p}$. II. 10 may be written in operator notation as

$$
\underline{H} F=S .
$$

F is uniquely determined by II.ll and specified boundary conditions.

Formally, the adjoint operator $\underline{H}^{+}$is defined by

$$
\left(\mathrm{F}^{+}, \underline{\mathrm{H} F}\right)=\left(\underline{\mathrm{H}}^{+} \mathrm{F}^{+}, \mathrm{F}\right),
$$


where $\vec{F}$ is any function obeying the boundary condition for the flux in II.li and F is any function obeying certaln boundary conditions which make II.12 hold. Here the Inner product, $(f, g)$ is the integral,

$$
(f, g)=\int f(\vec{p}) g(\vec{p}) d \vec{p}
$$

The normally present complex conjugation is absent because we are dealing with real functiona.

Now we consider the particular "adjoint flux": ${ }^{+}$ satisfying $\mathrm{H}^{+} \mathrm{F}^{+}=\mathrm{S}^{+}$, and satisfying the boundary conditions of the general $\mathrm{F}^{+}$In II.12: The adjoint source $\mathrm{S}^{+}$can be chosen arbitrarily. When $S=\left(\vec{p}-\vec{p}_{0}\right)$, the Dirac deltafunction, is substituted into II:i2,

$$
\left(F^{+}, \underline{H} F\right)=\left(F^{+}, S\right)=F^{+}\left(\vec{P}_{0}\right)
$$

Since

$$
\begin{aligned}
& \left.\underline{\mathrm{H}}^{+} \mathrm{F}^{+}, \mathrm{F}\right)=\left(\mathrm{S}^{+}, \mathrm{F}\right), \\
& \mathrm{F}^{+}\left(\overrightarrow{\mathrm{P}}_{\mathrm{O}}\right)=\left(\mathrm{S}^{+}, \mathrm{F}\right)
\end{aligned}
$$

F to, however, the Gieen's function, i.e., the tlux due to a untt source at $\overrightarrow{\mathrm{p}}_{0}$. Now if $\mathrm{s}^{+}$is taken to be the cross section for some physical process; then the adjoint 
flux at $\vec{p}_{0}$ is seen to be fust the rate at which the physical process represented by the cross section $\mathrm{s}^{+}$occurs throughout the system phase space when a unit source is introduced at $\vec{p}_{0}$. Any cross section like quantity can be used for the adjoint source and each one will give rise to a distinct adjoint solution. Stated another way, the adjoint flux is the probability that a neutron at $\vec{p}_{0}$ will eventualiy lead to: a process denoted by $\mathrm{s}^{+}$somewhere in the system phase space. Se69

With this interpretation of the adjoint flux it is clear that the function $F^{+}$defined in section II.A is the adjoint flux for the process of being absorbed within the LS core material. We turn now to the calculation of the adjoint flux and from it, via II.4 and II.5, $\sigma_{L S}(E)$.

The code ANISN, a one dimensional multigroup discrete ordinate transport code, can be used to calculate the adjoint flux with some changes in the input data and the interpretation of the angular flux: For a more detalled discussion of the ANISN method for calculating direct flux see Ref. La63 and $\operatorname{Tr} 68$. Here we w11l summarize the development of the group equations for adjoint flux with the details left to Appendix A. II.12 is found to be satisfied if the adjoint operation is

$$
\begin{aligned}
\underline{H}^{+} \mathrm{F}^{+}= & -\vec{\Omega} \cdot \nabla \mathrm{F}^{+}+\sum_{t} \mathrm{~F}^{+}-\iint \mathrm{d} \vec{\Omega} \cdot \mathrm{F}^{+} \cdot\left(\overrightarrow{\mathrm{r}}, \mathrm{u}^{\prime}, \vec{\Omega}^{\prime}\right) \times \\
& \times \Sigma_{\mathrm{S}}\left(\mathrm{u}+\mathrm{u}^{\prime} ; \vec{\Omega} \cdot \vec{\Omega}^{\prime}\right)
\end{aligned}
$$


with $F^{+}(\vec{r}, u, \vec{\Omega})=0$ for $\vec{\Omega} \cdot \vec{n}>0$, where $\vec{n}$ is the outward normal at the surface of the system. We first assume that the adjoint flux can be divided into $G$ energy groups, with group g extending from ${ }_{g}$ to ${ }_{g+i}$. Further, we assume that the groups are fine enough that the adjoint flux within each group may be taken as a constant whose value $\mathrm{F}_{\mathrm{g}}^{+}(r, \mu)$ is given by

$$
F_{g}^{+}(r, \vec{\Omega})=\int_{u_{g}}^{u_{g+i}} F^{+}(r, u, \vec{\Omega}) d u / \Delta u_{g}
$$

where $\Delta u_{g}=u_{g+1}-u_{g}$. The group adjoint flux is defined in this manner so that when $f t$ is multiplied by the group flux $F_{g}(\vec{r}, \vec{\Omega})$ (interpreted as belng the lintegral of $F(r, u, \vec{\Omega})$ over $u$ in group g) and integrated over $\vec{\Omega}_{0}$ and $\vec{\zeta}$ the saturated activity due to incident neutrons in g results.

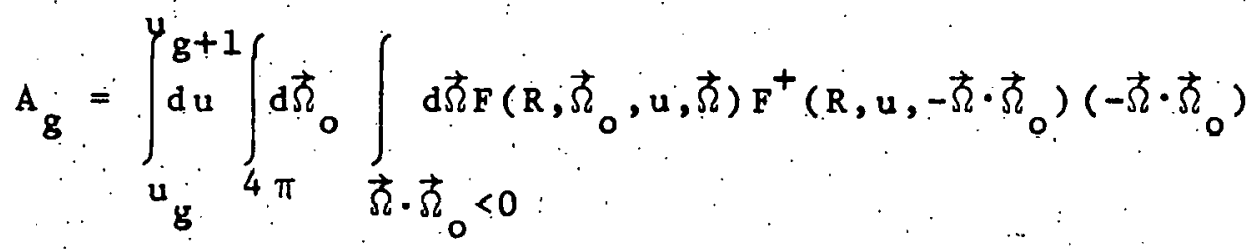

$$
\begin{aligned}
& A_{g} \simeq \int_{4 \pi} \mathrm{d} \vec{\Omega} \int_{0} \int_{\vec{\Omega} \cdot \vec{\Omega}} \mathrm{d} \vec{\Omega}_{\mathrm{g}}\left(R, \vec{\Omega}_{0}, \vec{\Omega}\right) \mathrm{F}_{\mathrm{g}}^{+}\left(\mathrm{R},-\vec{\Omega} \cdot \vec{\Omega}_{0}\right)\left(-\vec{\Omega}_{0} \cdot \vec{\Omega}_{0}\right)
\end{aligned}
$$

The total activity is then simply a sum over g .

$$
A=\sum_{g=1}^{G} A
$$


If we approximate the scattering kernel by an $L^{\text {th }}$ order Legendre expansion

$$
\sum_{S}\left(u^{\prime}+u ; \vec{\Omega}^{\prime} \cdot \vec{\Omega}\right)=\sum_{\ell=0}^{L} \frac{2 \ell+1}{4 \pi} B_{\ell}\left(u^{\prime}+u\right) P_{\ell}\left(\mu_{0}\right)
$$

then the adjoint kernel is

$$
\Sigma_{S}\left(u+u^{\prime} ; \vec{\Omega}^{\prime} \cdot \vec{\Omega}^{\prime}\right)=\sum_{\ell=0}^{\dot{S}_{l}} \frac{2 \ell+1}{4 \pi} B_{\ell}\left(u+u^{\prime}\right) P_{\ell}\left(\mu_{0}\right)
$$

where $\mu_{0}$ is the cosine of the angle between $\vec{\Omega}$ ! and $\vec{\Omega}$, and $B_{\ell}$ are determined from cross section data (see Appendix C). If we apply the spherical harmonics addition theorem and partially perform the integration, the adfoint equation with spherical symetry may be written as

$$
\begin{aligned}
& \left(-\mu \frac{\partial}{\partial r}-\frac{1-\mu^{2}}{r} \frac{\partial}{\partial \mu}+\Sigma_{t}(u)\right) F^{+}(r, u, \mu) \\
& -\sum_{\ell=0}^{L} \frac{2 \ell+1}{2} P_{\ell}(\mu) \int_{0}^{\infty} d_{u^{\prime}} B_{\ell}\left(u \rightarrow u^{\prime}\right) \int_{-1}^{1} d \mu^{\prime} P_{\ell}(\mu) F^{+}\left(r, u^{\prime}, \mu^{\prime}\right) \\
& =S^{+}(r, u, \mu) .
\end{aligned}
$$

Applying the operator

$$
\frac{1}{\Delta u} \int_{u_{g}}^{u} d u
$$


to II.14 we obtain

$$
\begin{aligned}
& \left(-\mu \frac{\partial}{\partial r}-\frac{1-\mu^{2}}{r} \frac{\partial}{\partial \mu}+\Sigma_{t g}\right) \mathbf{F}_{\mathbf{g}}^{+}(r, \mu)
\end{aligned}
$$

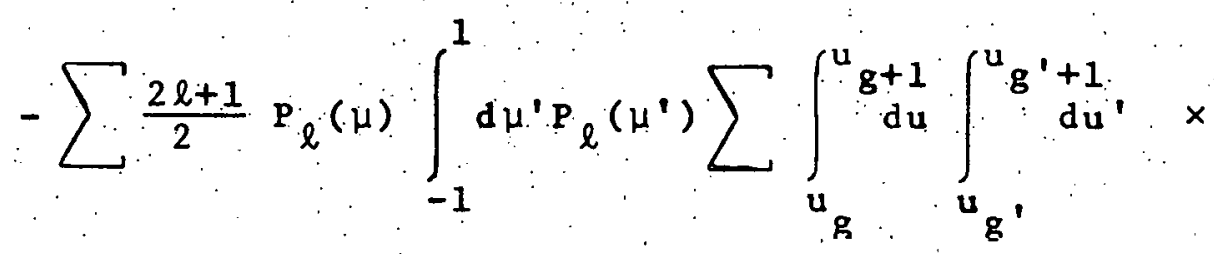

$$
\begin{aligned}
& \frac{B_{l^{\left(u \rightarrow u^{\prime}\right)}}}{\Delta u_{g}} \quad F^{+}\left(r, u^{\prime}, \mu^{\prime}\right) \\
& =\int_{u_{g}}^{u+d u s} s^{+}(r, u, \mu) / \Delta u_{g} \equiv s_{g}(r, \mu)
\end{aligned}
$$

Since $F_{g}^{+}\left(\vec{r}, \mu^{\prime}\right)$ ls Independent of u in group $g^{\prime}$

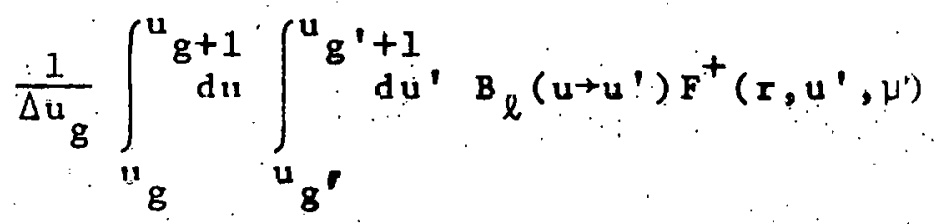

$$
\begin{aligned}
& =F_{g^{\prime}}^{+} \frac{1}{\Delta u_{g}} \int_{u_{g}}^{u_{g+1}} d u_{u_{g^{\prime}}}^{u_{g^{\prime}+1}{ }^{\prime} u^{\prime} B_{\ell}\left(u \rightarrow u^{\prime}\right)=B_{\ell} g^{+g^{\prime}} F_{g^{+}}{ }^{\prime}\left(r, \mu^{\prime}\right)}
\end{aligned}
$$

where

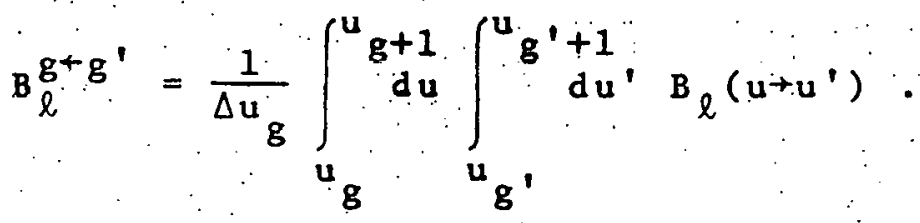

Thus, the scattering kernel for the adjoint problem is the 
transpose of the kernel for the forward problem. The multigroup approximation to II.14 then becomes

$$
\begin{aligned}
& \left(-\mu \frac{\partial}{\partial r}-\frac{1-\mu^{2}}{r} \frac{\partial}{\partial \mu}+\Sigma_{t g^{-}}\right) F_{g}^{+}(r, \mu) \\
& -\sum_{\ell=0}^{L} \sum_{g^{\prime}=1}^{G} \frac{2 \ell+1}{2} P_{\ell}(\mu) B_{\ell}^{g^{\prime}+g} \int_{-1}^{1} F_{g}^{+}\left(r, \mu^{\prime}\right) P_{\ell}\left(\mu^{\prime}\right) d \mu \\
& =S_{g}^{+}(r, \mu)
\end{aligned}
$$

Now if $-\mu$ is substituted for $\mu$ we obtain

$$
\begin{aligned}
& \left(\mu \frac{\partial}{\partial r}+\frac{1-\mu^{2}}{r} \frac{\partial}{\partial \mu}+\Sigma_{t g}\right) F_{g}^{+}(r,-\mu) \\
& \sum_{l=0}^{L} \sum_{g^{\prime}=1}^{G} \frac{2 l+1}{2} P_{\ell}(-\mu) B_{\ell}^{g^{\prime}+g} \int_{-1}^{1} d \mu^{\prime} F_{g}^{+}\left(r, \mu^{\prime}\right) P_{\ell}\left(\mu^{\prime}\right) \\
& =S_{g}(r,-\mu) .
\end{aligned}
$$

But

$$
\begin{aligned}
& P_{\ell}(-\mu) \int_{-1}^{1} \mathrm{~d} \mu^{\prime} \mathrm{P}_{\ell}\left(\mu^{\prime}\right) \mathrm{F}_{\mathrm{g}}^{+}\left(\mathbf{r}, \mu^{\prime}\right) \\
& =(-1)^{\ell} \mathrm{P}_{\ell}(\mu) \int_{-1}^{1} \mathrm{~d}^{\prime} \mathrm{P}_{\ell}\left(\mu^{\prime}\right)(-1)^{\ell} \mathrm{F}_{\mathbf{g}}^{+}\left(\mathbf{r},-\mu^{\prime}\right) \\
& =\mathrm{P}_{\ell}(\mu) \int_{-1}^{1} \mathrm{~d} \mu^{\prime} \mathrm{P}_{\ell}\left(\mu^{\prime}\right) \mathbf{F}_{\mathbf{g}}^{+}\left(\mathbf{r},-\mu^{\prime}\right)
\end{aligned}
$$


yielding

$$
\begin{aligned}
& \left(\mu \frac{\partial}{\partial r}+\frac{1-\mu^{2}}{r} \frac{\partial}{\partial \mu}+\sum_{t g}\right) F_{g}^{+}(r,-\mu) \\
& -\sum_{\ell=0}^{L} \sum_{g^{\prime}=1}^{G} \frac{2 \ell+1}{2} P_{\ell}(\mu) B_{l}^{g^{\prime}+g} \int_{-1}^{1} d \mu^{\prime} P_{\ell}\left(\mu^{\prime}\right) F_{g}^{+}\left(r,-\mu^{\prime}\right) \\
& =S_{g}^{+}(r,-\mu)
\end{aligned}
$$

which with the sigu of $\mu$ changed in $\mathrm{F}_{g}^{+}$and $\mathrm{s}_{g}^{+}$and transposition of the scattering kernel is identical to the equation solved numerically for the direct flux $F_{g}(r, \mu)$. Because the quadrature angles $\mu_{m}$ for the $s_{N}$ method to be used are chosen to be symmetric about $\mu=0$, the adjoint calculation of the leakage term proceeds as in the direct calculation; but Input for the angular dependent source $3_{g}^{+}\left(r, \mu_{m}\right)$ is entered as $S_{g}\left(r,-\mu_{m}\right)$, and the resulting flux $F_{g}\left(r, \mu_{m}\right)$ is interpreted as the adjoint flux $F_{g}^{+}\left(r,-\mu_{m}\right)$ : For example the boundary condition of no incoming flux in an adjoint problem is interpreted as a condition of no outgolng flux.. In addition to transposing the scattering matrix, we reverse the group order of the cross sertions; the source, and the starting flux guess. "This inversion is convenient because the adjoint of a downsatter probiem is an upscatter problem, which takes more time to compute. By proceeding in inverse group order, the upscatter problem becomes once more a downscater problem. 
Now if the macroscopic absorption cross section $\Sigma_{a}$ of the core material is a smooth function of energy, the adjoint flux can be calculated accurately by means of the group adjoint source:

$$
S_{g}^{+}(r)=\sum_{a g} H\left(R_{c o r} e^{-r}\right)=H\left(R_{c o r} e^{-r)} \int_{u_{g}}^{u} g+1 \frac{\sum_{a}(u) d u}{\Delta u_{g}}\right.
$$

where His the Heavyside step-function and $R_{\text {core }}$ is the core radius. This method is useful at high energies where the absorption cross section is slowly varying. At lower energies ( 1 to $1000 \mathrm{ev}$ ) the cross section exhibits a resonance structure (the property we are trying to exploit) and to calculate adjoint flux reasonably accurately in this region would require an inordinately large number of groups. Because of the large cross section values within a resonance, the spatial mesh within the core would have to beextremely small to account for the very short mean free path. Tests (see Appendix B) have shown that calculations become unreliable when the mesh spacing becomes as large as a mean free path. Thus, some other method is required by computer space and time limitations.

If one considers the capture probability for neutrons entering the core, it is seen (Figure 9) that it is a more slowly varying function of energy than the resonance cross gectloirs, so that tis gruup áveraged value will yield 
more accurate results for the important low energy resonances. The idea here is to calculate the adjolnt flux (capture probability) for a bare core and use the result as a boundary condition, leaving to the code the calculation of this adfoint flux through the cadmium, polyethylene and boron ( 1 it is present). The advantage of this method is that one set of boundary conditions for a given core material may be used for many different polyethylene-boron configurations. This scheme neglects the probability of a neutron being absorbed in the core if it is heading out. It will be shown in section D that this approximation does not result in any. serlous error.

The adjolnt flux boundary condition is calculated on the basis of a first flight capture probability with a second flight correction term. Since an ANISN interpretation of the adjoint flux at a Legendre-Gauss quadrature angle $\mu_{\overline{\mathbf{m}}} 1 \mathbf{R}$

$$
F^{+}\left(r, u, \mu_{m}\right)=\sum_{\ell=0}^{L} \frac{2 \ell+1}{2} Q_{\ell}(r, u) P_{\ell}\left(\mu_{m}\right)
$$

1t. is desirable to expand the capture probability in Legendre polynomials to remain consistent. This is simple for the first flight capture probability $\mathrm{F}_{1}^{+}\left(u_{0}, \mu\right)$, whlch is given by

$$
F_{1}^{+}\left(u_{0}, \mu\right)= \begin{cases}\frac{\Gamma_{c}\left(u_{0}\right)}{\Sigma_{t}\left(u_{0}\right)}\left(1-e^{+2 \mu R \Sigma_{t}\left(u_{0}\right)}\right) & \mu<0 \\ 0 & \mu>0\end{cases}
$$


where $u_{0}$ is the incident neutron lethargy, $R$ is the core radius, $\mu$ is the cosine of the angle of the neutron with respect to the outward normal (see Figure 7 ), $\Sigma_{t}\left(u_{0}\right)$ is the total macroscopic cross section, and $\Sigma_{c}\left(u_{o}\right)$ is the macroscopic absorption cross section for a process which leads to a countable radioactive nucleus. The Legendre coefficient $Q_{\ell}\left(u_{0}\right)$ is given by

$$
\begin{aligned}
& Q_{\ell}\left(u_{0}\right)=\int_{-1}^{0} P_{\ell}(\mu) F_{1}^{+}\left(u_{0}, \mu\right) d \mu+\int_{0}^{1} P_{\ell}(\mu) \cdot 0 d \mu \\
& =\int_{-1}^{0} P_{\ell}(\mu) \frac{\Sigma_{c}}{\Sigma_{t}}\left(1-e^{2 \mu R \Sigma} t\right) d \mu
\end{aligned}
$$

Since $P_{\ell}(\mu)=\frac{P_{\ell+1}^{\prime}(\mu)-P_{\ell-1}^{\prime}(\mu)}{2 \ell+1}$,

$$
Q_{\ell}\left(u_{0}\right)=\int_{-1}^{0} \frac{P_{\ell+1}^{\prime}(\mu)-P_{\ell-I^{\prime}}^{\prime}(\mu)}{2 \ell+1} \frac{\Sigma_{c}}{\Sigma_{t}}\left(1-e^{2 \mu \Sigma_{t}^{R}}\right) \mathrm{d} \mu
$$

Integrating by parts yields

$$
\begin{aligned}
& \left.Q_{\ell}\left(u_{0}\right)=\frac{P_{\ell+1}(\mu)-P_{\ell-1}(\mu)}{2 \ell+1} \cdot \frac{\Sigma_{c}}{\Sigma_{t}}\left(1-e^{2 \mu \Sigma_{t} R}\right)\right]_{-1}^{0} \\
& +\frac{2 \Sigma_{t} R}{2 \ell+1} \frac{\Sigma_{c}}{\Sigma_{t}}\left[\int_{-1}^{0} d \mu P_{\ell+1}(\mu) e^{2 \mu \Sigma_{t} R}-\int_{-1}^{0} d \mu P_{\ell-1}(\mu) e^{\left.2 \mu \Sigma_{t} R\right]}\right.
\end{aligned}
$$




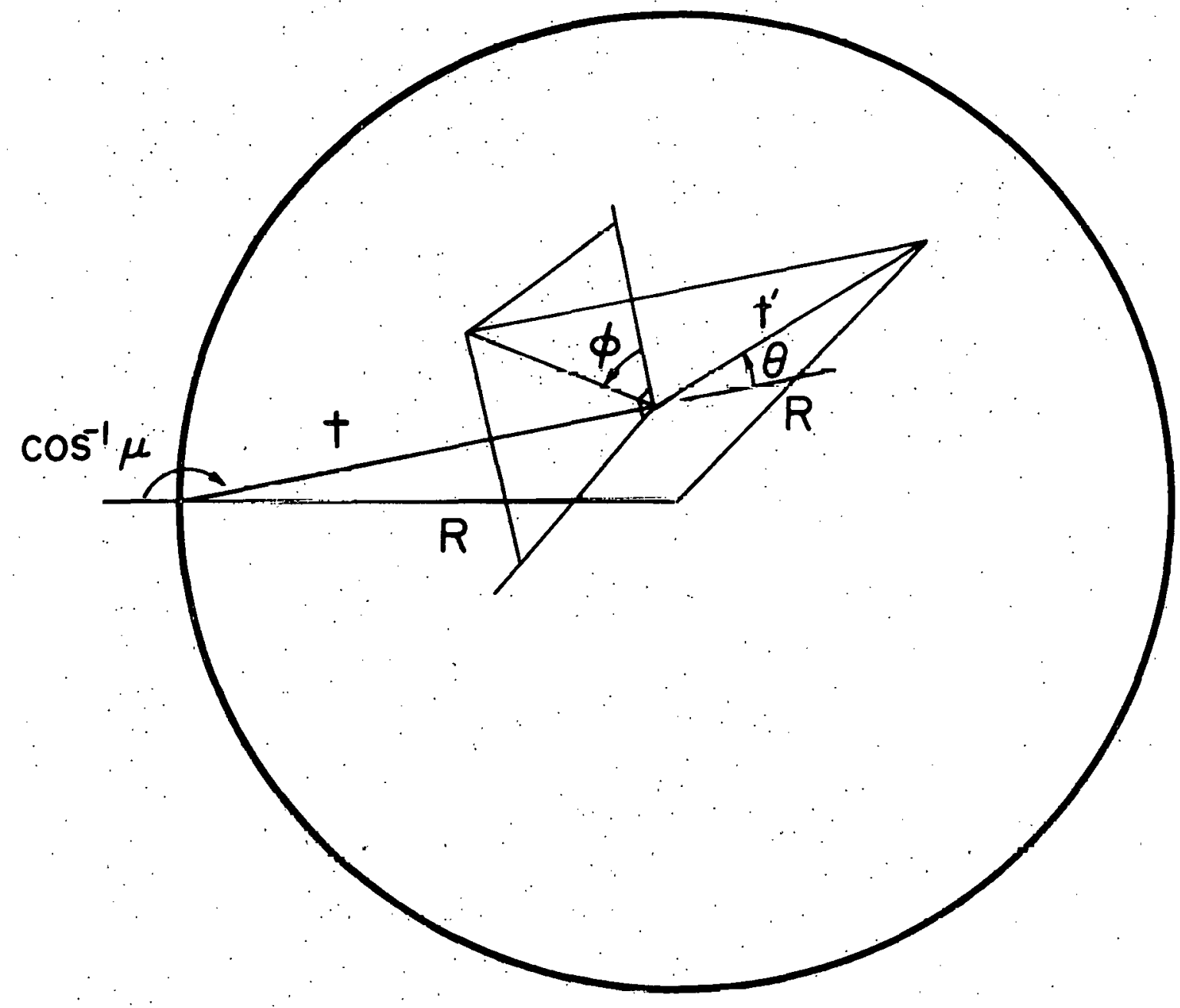

FIGURE 7. Geometry for Calculating Core Capture Probability. The center of the sphere. and $t$ are in the plane of the paper. The. intersection of $t$ and $t^{\prime}$ is the origin of a spherical coordinate system. $\theta$ and $\phi$ are the polar and azimuthal angles respectively. 
The first term on the right hand side vanishes leaving

$$
\begin{aligned}
& Q_{\ell}\left(u_{0}\right)=\frac{2 \Sigma_{t} R}{2 \ell+1}\left\{Q_{\ell-1}\left(u_{0}\right)-Q_{\ell+1}+\frac{\Sigma_{c}}{\Sigma_{t}} \times\right. \\
& \vdots \\
& \left.\int_{-1}^{0} d \mu\left[P_{\ell+1}(\mu)-P_{\ell-1}(\mu)\right]\right\} .
\end{aligned}
$$

Solving for $Q_{\ell+1}$ gives

$$
Q_{\ell+1}\left(u_{0}\right)=Q_{\ell-1}\left(u_{0}\right)-\frac{2 \ell+1}{2 \sum_{t}\left(u_{0}\right)} Q_{\ell}\left(u_{0}\right)+C_{\ell+1}\left(u_{0}\right)
$$

with

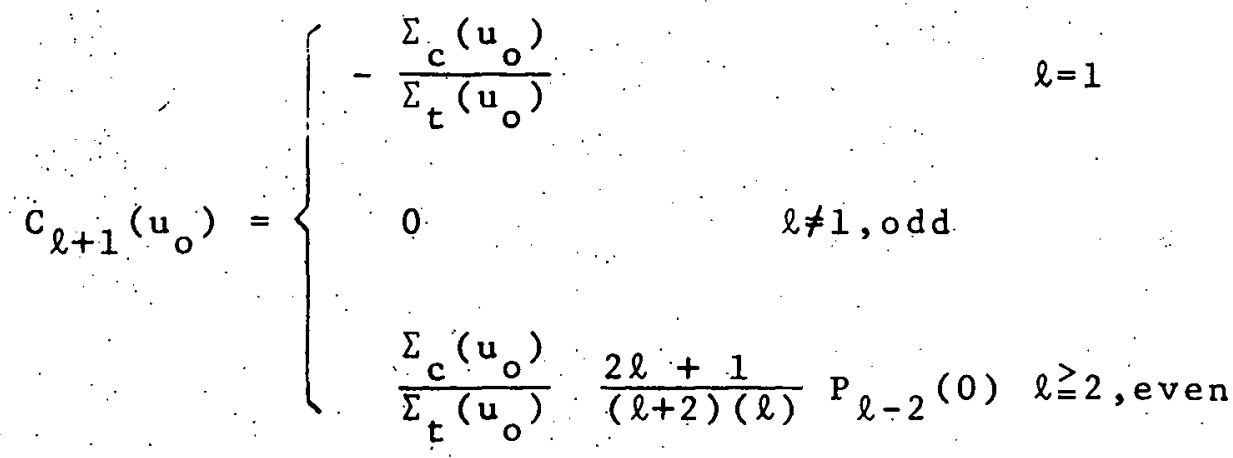

$$
\begin{aligned}
& \dot{Q}_{0}\left(u_{0}\right)=\frac{\Sigma_{c}\left(u_{0}\right)}{\Sigma_{t}\left(u_{0}\right)}\left[1+\frac{e^{-2 R \Sigma_{t}\left(u_{0}\right)}-1}{2 R \Sigma_{t}\left(u_{0}\right)}\right]
\end{aligned}
$$

and

$$
\begin{aligned}
& Q_{1}\left(u_{0}\right)=\frac{\Sigma_{c}\left(u_{0}\right)}{\Sigma_{t}\left(u_{0}\right)}\left[\frac{1}{\left(2 R \Sigma_{t}\left(u_{0}\right)\right)^{2}}-\frac{1}{2}-\frac{e^{-2 R \Sigma_{t}\left(u_{0}\right)}}{2 R \Sigma_{t}\left(u_{0}\right)} \times\right. \\
& \left.\left(1+\frac{1}{2 \cdot R \Sigma_{t}\left(u_{o}\right)}\right)\right]
\end{aligned}
$$


The recursion relationship for $Q_{\ell}\left(u_{0}\right)$ allows it to be numerically averaged over each group in the resonance region, and the first flight boundary condition to be generated with the help of II. 20 .

A second filght correction was added to account for slowing down within the core. This: is given by

$$
\begin{aligned}
F_{2}^{+}\left(u_{0}, \mu\right)= & \int_{0}^{2 \mu R} d t \int_{0}^{2 \pi} d \phi \int_{0}^{\pi} d \theta \Sigma_{S}\left(u_{0}\right) e^{-\Sigma_{t}\left(u_{0}\right) t} x \\
& \frac{\Sigma_{c}(u(\theta))}{\Sigma_{t}(u(\theta))}\left[1-e^{-\Sigma_{t}(u(\theta)) t^{\prime}(\theta ; \phi, t)}\right]
\end{aligned}
$$

where $\Sigma_{s}\left(u_{0}\right)$ is the scattering cross section; $\theta$ and $\phi$ determine the direction of the scattered neutron; and $t$ is the escape path for a neutron that scattered at $t$ (see Figure 7):

$$
\begin{aligned}
& t^{\prime}=-\eta(t+R \mu)+R \cos \phi \sqrt{1-\eta^{2}} \sqrt{1-\mu^{2}}+ \\
& \quad+\sqrt{\left[\eta\left(t-R \mu-R \cos \phi(1-\mu)^{1 / 2}(1-\eta)^{1 / 2}\right]^{2}-t(t-2 R \mu)\right.}
\end{aligned}
$$

where n-cus $\theta$. . The secondary lethargy $u$ is determined from kinematics of the scattering process as

$$
u(\theta)=u_{0}-\ln \left[\frac{A^{2}+2 A \cos \theta+1}{(A+1)^{2}}\right]
$$


where $A$ is the mass of the core material. It is assumed here that the $1 a b$ and center of mass scattering angles are equal for heavy nuclei. $F_{2}^{+}$was simply evaluated at each quadrature angle and added to the first flight contribution. Its effect was small (accounting for only $2.9 \%$ of the adjoint "source", see Figure 8), and expansion in Legendre polynomials would have required an additional numerical integration. The triple integral was evaluated using an elght point Gauss Legendre scheme and then averaged over lethargy according to Simpson's rule.

The boundary condition for the adjoint flux at the Gauss Legendre quadrature angles $\mu_{m}$ is thus

$$
F_{g}^{+}\left(R_{c o r e} \mu_{m}\right)=\sum_{\ell=0}^{L} \frac{2 \ell+1}{2} Q_{\ell g} P_{\ell}\left(\mu_{m}\right)+F_{2 g}^{+}\left(R_{c o r e} \mu_{m}\right)
$$

where the subscript $g$ implies an average over the $g^{\text {th }}$ group and $R_{\text {core }}$ is the core radius.

By means of a combination of this boundary condition at low energies. and an absorption cross section adjoint source (II.19) at high energies the adjoint flux at the surface of the. LS can be determined by the code ANISN.

D. Description of Input Data and Test Calculations

The group scheme for the calculation of $\sigma_{L S}(E)$ was chosen to be a. 74-group structure. It was formed by collapsing the GAM Ad67 99-group structure (Table 2) for which cross section libraries exist. The number of groups 
TABLE 2

99-GROUP GAM STRUCTURE

TROUP BOUNDARIES

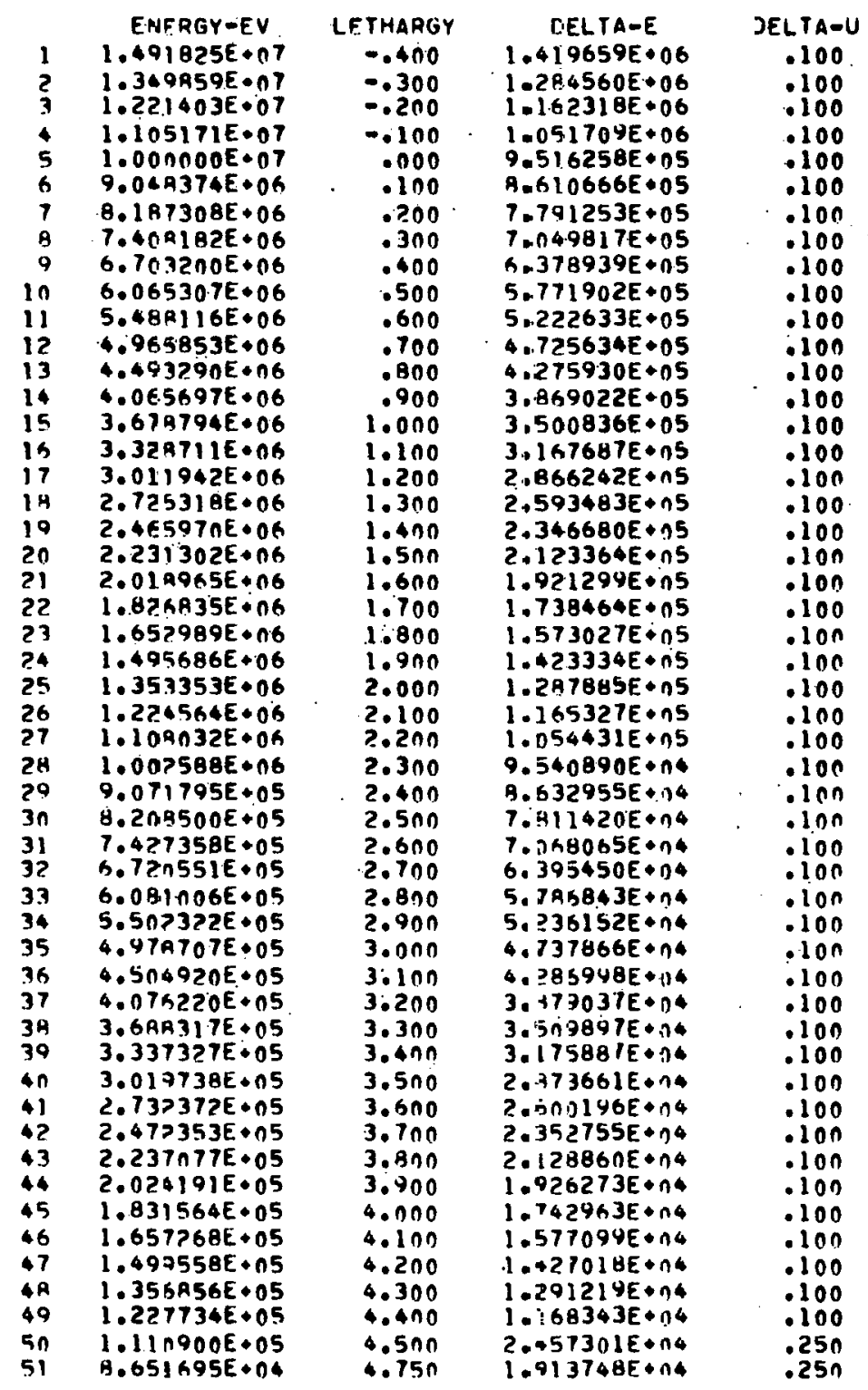

\begin{tabular}{|c|c|c|c|}
\hline 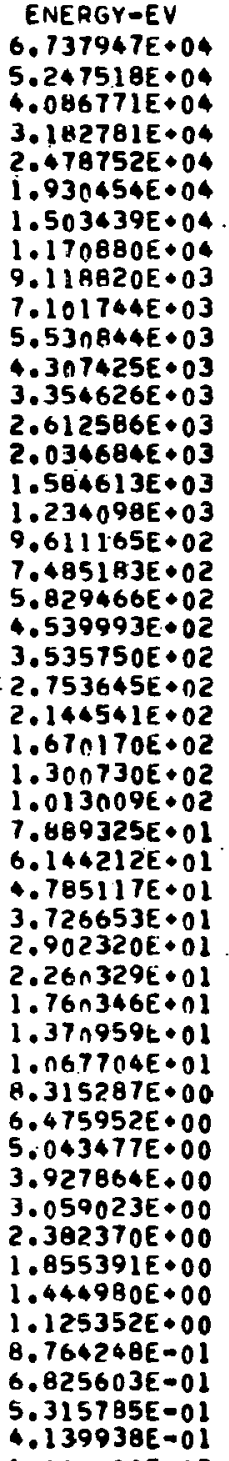 & $\begin{array}{r}\text { LETHARGY } \\
5.000 \\
5.250 \\
5.500 \\
5.750 \\
6.000 \\
6.250 \\
.6 .500 \\
6.750 \\
7.000 \\
7.250 \\
7.500 \\
7.750 \\
8.000 \\
8.250 \\
8.500 \\
8.750 \\
9.000 \\
9.250 \\
9.500 \\
9.750 \\
10.000 \\
10.250 \\
10.500 \\
10.750 \\
11.000 \\
11.250 \\
11.500 \\
11.750 \\
12.000 \\
12.250 \\
12.500 \\
12.750 \\
13.000 \\
13.250 \\
13.500 \\
13.750 \\
14.000 \\
14.250 \\
14.500 \\
14.750 \\
15.000 \\
15.250 \\
15.500 \\
15.750 \\
16.000 \\
16.250 \\
16.500 \\
16.750 \\
17.000\end{array}$ & 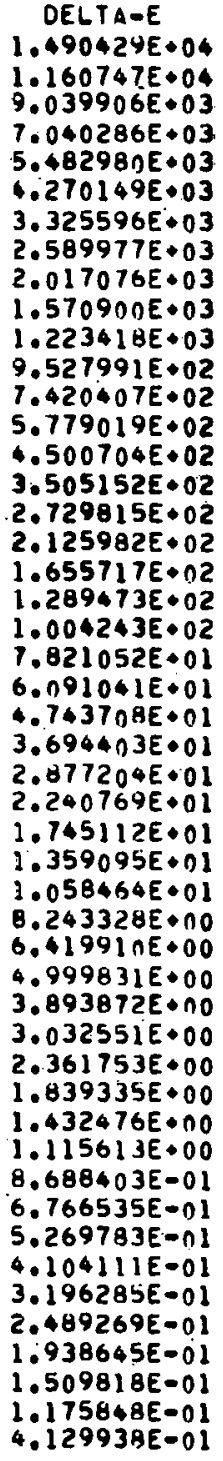 & $\begin{array}{r}\text { DELTA-U } \\
.250 \\
.250 \\
.250 \\
.250 \\
.250 \\
.250 \\
.250 \\
.250 \\
.250 \\
.250 \\
.250 \\
.250 \\
.250 \\
.250 \\
.250 \\
.250 \\
.250 \\
.250 \\
.250 \\
.250 \\
.250 \\
.250 \\
.250 \\
.250 \\
.250 \\
.250 \\
.250 \\
.250 \\
.250 \\
.250 \\
.250 \\
.250\end{array}$ \\
\hline
\end{tabular}


was reduced in the interest of shortening computation time. Group 2 through 49 of width.1 lethargy units (1.u.) of the GAM-II structure were combined to form 24 groups of width.2 1.u.; the remaining 50 groups were left unchanged. The resulting scheme is given in Table 3 . A cross section $\sigma_{x i}^{\prime}$ for the new group $i$ was given by

$$
\begin{array}{ll}
\sigma_{x i}^{\prime}=\frac{\sigma_{x, 2 i}+\sigma_{x, 21+1}}{2} & i=1, \ldots, 24 \\
\sigma_{x 1}^{\prime}=\sigma_{x, 1+25} & i=25, \ldots, 74
\end{array}
$$

where $x$ stands for elther total or absorption. The expansion coefficients for the scattering kernel $\mathrm{B}_{\ell}^{j+i}$ was given by

$$
\begin{aligned}
& B_{\ell}^{j+1 !}=\frac{B_{\ell}^{2 i+21}+B_{\ell}^{2 i+21+1}+B_{l}^{21+1+2 i+1}}{2} \quad \begin{array}{l}
i=j \\
i=1, \ldots, 24
\end{array} \\
& =\frac{B_{\ell}^{2 j+21}+B_{\ell}^{2 j+1+21}+B_{\ell}^{2 j+2 i+1}+B_{\ell}^{2 j+1+21+1}}{2} \\
& i=1, \ldots, 24 \\
& j=25, \ldots, 74 \\
& =\frac{B_{\ell}^{j+25+21}+B_{\ell}^{j+25+21+1}}{2} \\
& ={ }^{j}{ }_{\ell}^{j+25+i+25} \\
& i=1, \ldots, 24 \\
& j=25, \ldots, 74 \\
& i=25, \ldots, 74 \\
& j=25, \ldots, 74
\end{aligned}
$$

Remember that in a downscatter problem

$$
B_{\ell}^{j+1}:=0
$$


GROUP. BOUNDARIES

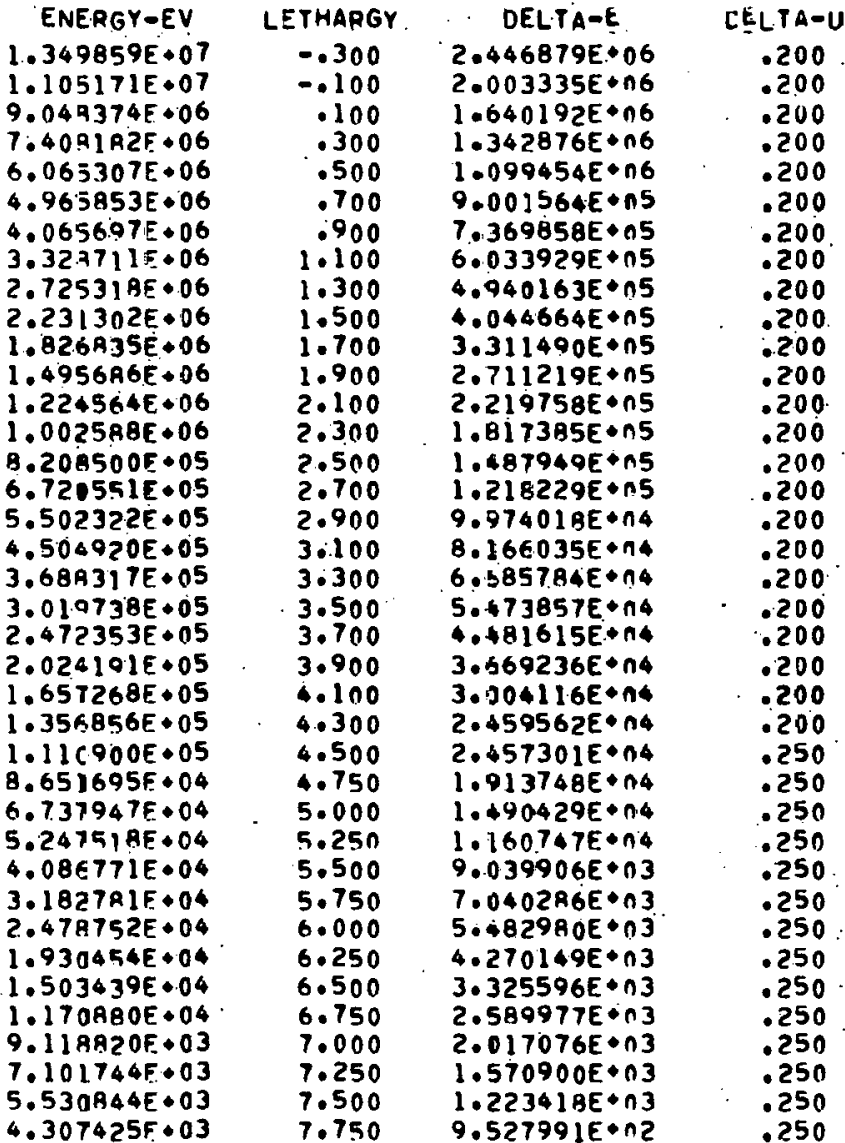

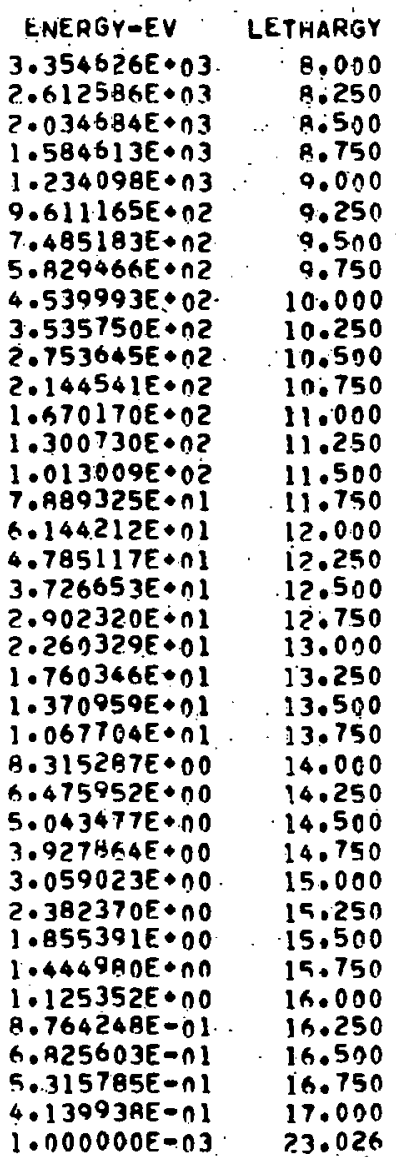

DELTA-E

7.420407E+02 $5.779019 E+05$ $4.5007045 \cdot n$ ?

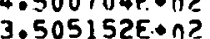
(3. (1.) $125982 E \cdot n 2$ $1.5594735+055$ $1.004243 E+02$ $7.821052 E+n 1:$ $6.091041 E \cdot 01$ $4.74370 B E \bullet 01$ $3.694403 E \cdot 0$ 2.877204E+nI 2.2407605 . 1.2451.12E.01 $1.359095 E+01$ $1.359095 E+0$ 1.053342 ? $6.419910 E+00$ $3.093872 E$ O $3.893 \mathrm{~B}$ 2E $\mathrm{nO}$ $3.032551 E \cdot 00$ $2.361753 E \cdot n$. I. B39335E•nO $1.43247 .6 E+n n$ $1.115613 E+n O$ 8. 68R40.3F-n h.766535E-n 5.269783F $=n 1$ $4.104111 E-n$ 3.1962B5E-n $2.4892695=n$ $1.9386455-n$ $1.50981 \mathrm{AE}-n$ $1.175 A 4 B E-n !$
$4.12993 A E-n$
DELTA-U .250 .250
.250 .250 .250
.250 .250 .250
.250 .250 .250 .250 .250 .250
.250 .250 .250
.250 .250 .250. .250 . .250 .250 .250 . .250 .250 .250 .250 .250 .250 .250 .250 .2 .50 
Cross sections for hydrogen and carbon for the polyethylene were from the DLC-2, set. ${ }^{\dagger}$ Boron and cadmium cross-sections were from the "fast" library for the code GGC-4: ${ }^{+t}$ These fine group sets were generated from energy dependent data by methods similar to those summarized in Appendix.C. The speciflc gravity of the polyethylene $\left(\mathrm{CH}_{2}\right)$ was given by the manufacturer as .93 , leading to an atom density of .04 atoms $/ \mathrm{cm}^{3}$. The density of boron carbide ( ${ }_{4} \mathrm{C}$, the form of boron selected for design) was chosen equal to that which could be attained by packing $\mathrm{B}_{4} \mathrm{C}$ powder (approximately $1 / 2$ theoretical density) into a spherical shell surrounding the polyethylene (see Figure 5).

In what follows, a four character code $(x, x, y, z)$ 1s followed for the structure of a given spectrometer. $X$ is the core material, $x$ is the core diameter in $m, y$ is the polyethylene diameter in inches, and $z$ is the $\mathrm{B}_{4} \mathrm{C}$ powder thickness $(\Delta r)$ in inches (density assumed to be $\left.1.40 \mathrm{grams} / \mathrm{cm}^{3}\right)$. In all cases the cadmium thickness was $40 \operatorname{mil} .$.

As can be seen in equations. II: $20-26$, the total cross section $\Sigma_{T}(E)$, the scattering cross section $\Sigma_{S}(E)$, and an activation cross section $\Sigma_{c}(E)$ are required for the deterination of the adjoint flux boundary condition at the polyethylene-core interface. The materials chosen for the core were natural indium, gold, and manganese. The most

\footnotetext{
Tobtalned from the Radiation Shielding Information Center at oak Ridge National Laboratory.

††From the Argonne code center.
} 
extensive calculations were performed for indium since they were partially tested experimentally (see Chapter III). Natural indium consists of two isotopes 113 In and 115 In, with abundances $4 \%$ and $96 \%$ respectively. Resonance parameters ${ }^{6066}$, from which the total and scattering cross sections for natural indium and the absorption cross section for ${ }^{115}$ In can be calculated (Appendix $c$ ), have been determined for neutron energies up to $100 \mathrm{eV}$. When ${ }^{115}$ In absorbs a neutron and the highly excited ${ }^{116}$ In compound nucleus Is formed, three possible final states of ${ }^{116}$ In result from the $\gamma$-decay that follows. Dr58,Do60,Po65 one is a convententiy counted 54 min isomeric state ${ }^{116} \operatorname{In}(\mathrm{m} 1)$, and the other two are the $14 \mathrm{sec}$ ground state and a 2 second isomeric state. ${ }^{116} \operatorname{In}(\mathrm{m} 2)$. The probability of being in the 54 min state depends upon the incident neutron energy and the J-value of the compound nucleus. This probability has been determined for thermal energies and the first three resonances $(1.46,3.86$, and $9.12 \mathrm{eV})$. over this range it is a constant $p_{0}$ except in the neighborhood of the $3.86 \mathrm{eV}$ resonance where a minimum $\mathrm{P}_{\text {II }}$ is attained. The average value of this probability was found to be close to $p_{0}$ from resonance tategral measurements. Be63,A165 Here it is somewhat arbitrarily assumed that

$$
\Sigma_{c}(E)=\Sigma_{116} \operatorname{In(m1)}(E)=\Sigma_{a}^{116} \operatorname{In}(E)\left\{p_{0}-\frac{p_{0}-p_{I I}}{1-\frac{4(E-3.86)^{2}}{\Gamma^{2}}}\right\}
$$


up to $10 \mathrm{keV}$, where $\Gamma$ is the total width of the 3.86 resonance, and $p_{0}$ and $p_{I I}$ are .789 and .661 respectively. Using II. 26-27 and the resonance parameters, the group average boundary condition $\mathrm{F}_{\mathrm{g}}^{+}\left(\mathrm{R}_{\text {core }}, \mu\right)$ was calculated for a $4 \mathrm{~mm}$ diameter natural indium sphere for the energy groups between the cadmium cut-off and 61 ev. Above this energy accurate pointwise data were not avaliable, and group average cross sections were used both as an adjoint source, as in II.19, or directly. In II.26, to calculate the adjoint boundary condition. These two techniques" gave the same results for $\sigma_{L S}(E)$ (to within . $73 \%$ ) and were therefore assumed to be equivalent. Considerable computation time was saved by using the adjoint boundary condition, so it was selected for most of the calculations.

From 61 eV to $10 \mathrm{keV}$ the average cross sections needed were obtained from the "fast" library for the Code GGC-4, which were generated from average resonance parameters in a manner simflar to that mentioned in Appendix C. Above $10 \mathrm{keV}$ the cross section for the formation of ${ }^{116} \operatorname{In}(\mathrm{m} 1)$ and others needed have been measured. G066 Fortunately no such complications arise for gold and manganese: the $(n, \gamma)$ process ends in the ground state of the compound nucleus; and the cross sections needed are available in evaluated form. ${ }^{\dagger}$ The methods used and the input data sources for the calculation of $\mathrm{F}_{\mathrm{g}}^{+}\left(\mathrm{R}_{\text {core }}, \mu\right)$ are summarized

From the ENDF/B (Evaluated Nuclear Data File) CSEWG. 
in Table $4 \%$

Results for $\sigma_{\text {core }}$ (the core counterpart to $\sigma_{L S}$;

see II.4 and II.5) for Indium are given in Figures 8 and

9. Included in Figure 8 is a plot of $\sigma_{\text {core }}^{(E)}$ for comparison.

It can be seen that the principal ( 1.45 eV) resonance is

very well approximated by the group average value. This

is important since this resonance accounts for $70 \%$ of the area under the curve and hence 18 the major contribution

to the absorption probability. Figure 10 gives $F_{g}^{+}(\mu)$

for several groups. Here $\mathrm{F}_{g}^{+}(\mu)$ is expanded in Legendre

Polynomials up to order 7. The four angles for the ANISN

boundary condition for an $\mathbf{8} 8$. (eighthorder Gauss-Legendre

Quadrature) approximation are indicated. It should be

noted that the black core value is not 1.0 but .789 since

only this fraction of the neutrons captured lead to the

54 min isomer of ${ }^{116} \mathrm{In}$.

Tests of the code ANISN were made to determine mesh spacing, order of angular quadrature and order of the scattering kernel that would give good results. Several problems for which analytic solutions exist were run (sep Appendix B), and thcae showed lhat a mash spacing of $1 / 10$ the shortest mean free path always gave accurate results for the spatial dependence. Calculations were performed for various quadrature and scattering-kernal orders to determine if the $P-7$ kernel avallable would be adequate. The smallest polyethylene sphere contemplated 
TABLE 4

SUMMARY OF METHODS USED FOR ADJOINT SOURCE USED IN ANISN

\begin{tabular}{|c|c|c|}
\hline $\begin{array}{l}\text { Áctivation Process } \\
\therefore\end{array}$ & $\begin{array}{l}\text { Resorance Region } \\
\mathrm{F}_{\mathrm{g}}^{+}=\int_{\mathrm{g}}^{\mathrm{F}^{+}}(\mathrm{E}) \mathrm{dE}\end{array}$ & Average Cross Sections Used \\
\hline $\begin{array}{c}\operatorname{In}(\mathrm{n}, \gamma)^{116} \operatorname{In}(\mathrm{m} 1) \\
54 \mathrm{~m}\end{array}$ & $\begin{array}{c}.4-61 \text { eV cross sections } \\
\text { from Go and Eq. II.27 } \\
\text { a } 26 \text { a }\end{array}$ & $\begin{array}{l}\text { Either } F_{g}^{+} \text {from } \bar{\Sigma}_{g}(E q . \text { II.26) or } \\
S_{g}^{+}=\Sigma_{c g}(E q . I I .19) \\
61 \text { eV }-10 \mathrm{keV} \mathrm{"fast"} \mathrm{data} \mathrm{for} \\
\text { GGC-4 and Eq. II. } 27 \\
10 \mathrm{keV}-13.5 \mathrm{MeV} \text { average cross } \\
\text { sections calculated from data in } \\
\text { Go } 66 \text { combined with "fast" data } \\
\text { for GGC-4 }\end{array}$ \\
\hline $\begin{array}{c}197 \mathrm{Au}(\mathrm{n}, \gamma){ }^{198 \mathrm{Au}} \\
2.7 \mathrm{~d}\end{array}$ & $\begin{array}{l}\because 4-960 \text { eV recent eval- } \\
\text { uation from ENDF/B } \\
\text { file }\end{array}$ & $\begin{array}{l}960 \mathrm{eV}-13.5 \mathrm{MeV} \text { average cross } \\
\text { sections from DLC-2 set (which was } \\
\text { generated from ENDF/B data) used } \\
\text { to calculate } \mathrm{F}_{\mathrm{g}}^{+} \text {(Eq. II.26) }\end{array}$ \\
\hline $\begin{array}{l}{ }^{5.5} \mathrm{Mn}(\mathrm{n}, \dot{\gamma}){ }^{56} \mathrm{Mn} \\
2.56 \mathrm{~h}\end{array}$ & & $.4 \mathrm{eV}-13.5 \mathrm{MeV}$ same as for gold \\
\hline
\end{tabular}




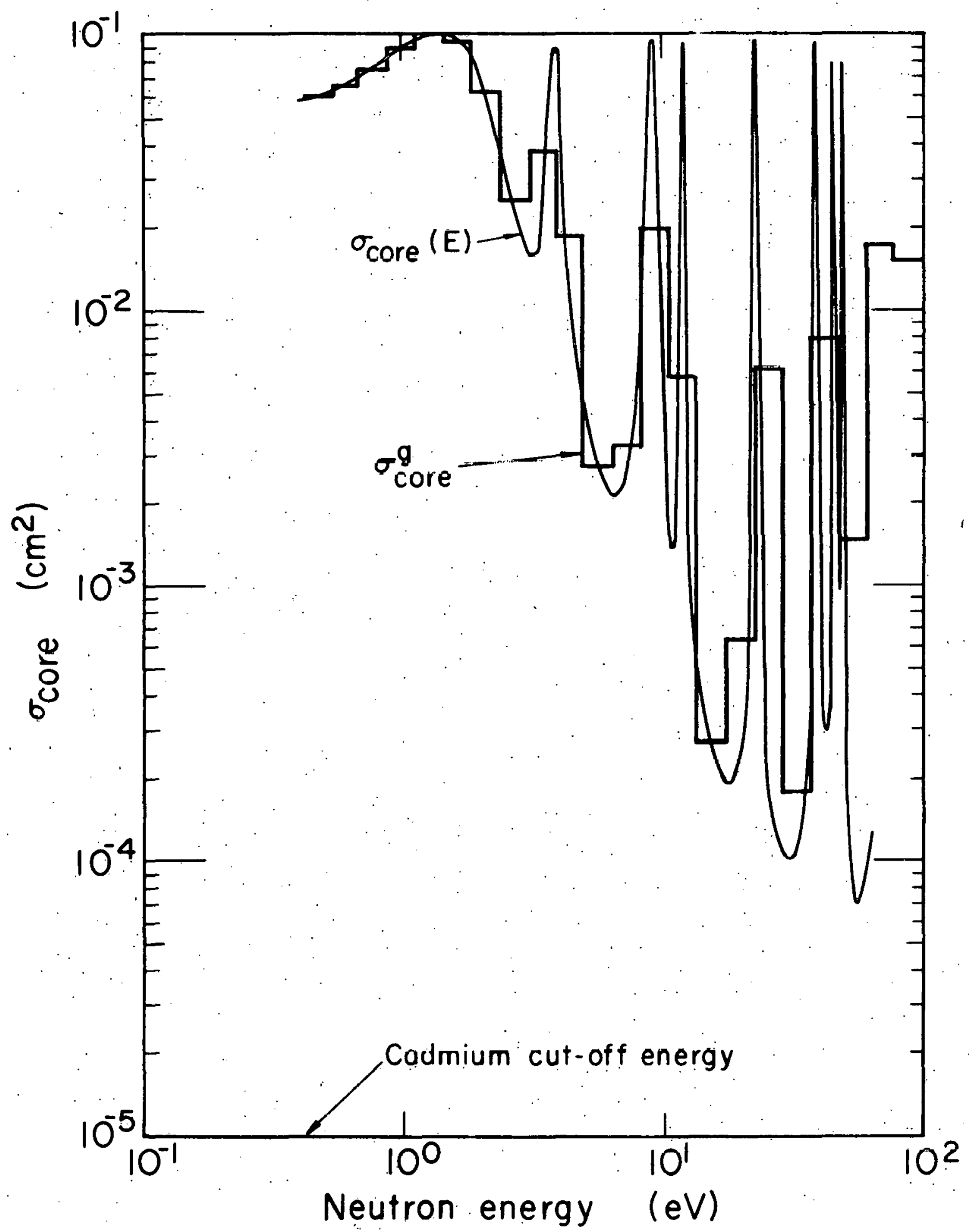

XBL $7012-4209$

FIGURE 8. Effective Cross Section of $4 \mathrm{~mm}$ Indium Core; from .1 to $100 \mathrm{eV}$. The superscript $g$ denotes group average. 


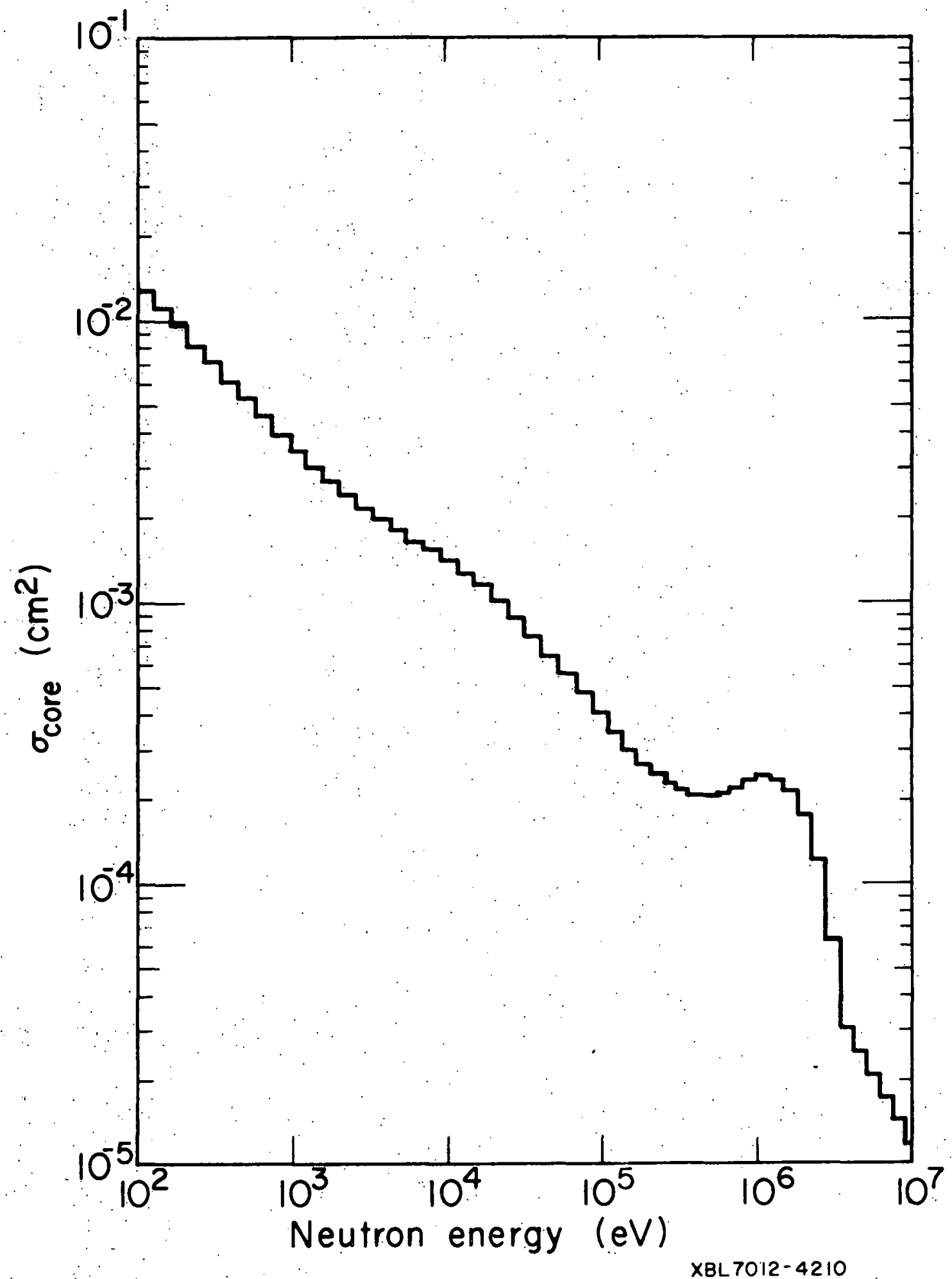

FIGURE 9. Effective Cross Section of a $4 \mathrm{~mm}$ Diameter Indium Core, from $10^{2}$ to $10^{7} \mathrm{eV}$. 


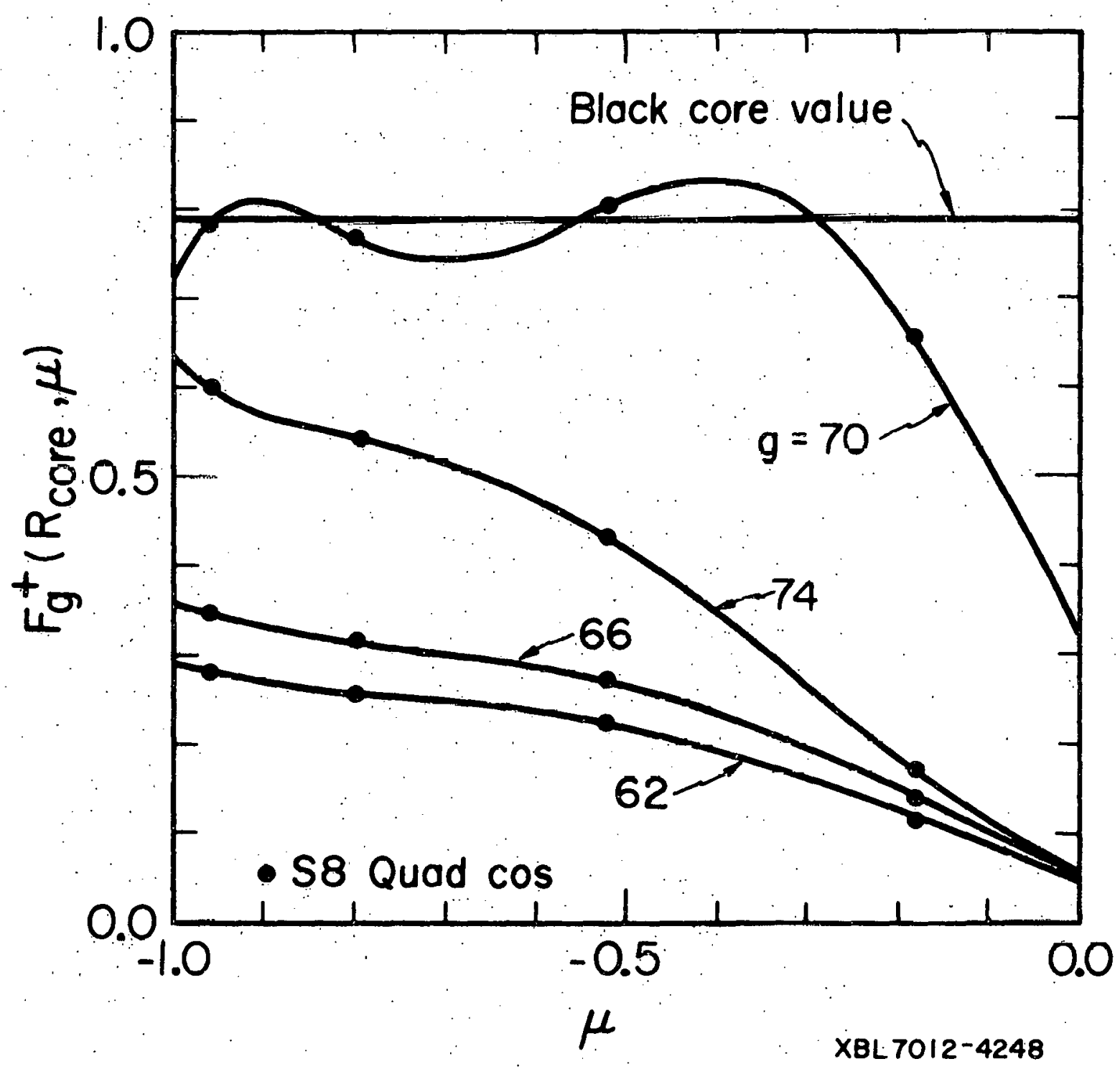

FIGURE 10. $F_{g}^{+1}\left(R_{\text {core }}, \mu\right)$ for In Core for $g=62,66,70,74$ (groups numbered as in. Table 3 ) 
(In $4,2,0$ ) was chosen for consideration since it was

thought to be the most sensitive:

Results are given in Figure 11: As the order is increased, the peak response value increases and shifts to lower energy. The change between $P-7$ and $P-3$ is less than the change between $P-3$ and $P-1$, indicating that higher order terms will have a small effect. For the P-7 kernel the difference between. $\$ 8$ and 512 quadrature is small compared to the errors due to inaccuracies in the indium cross section. The variation for two larger spheres (In $4,4,0$ and In $4,3,1$ ) are given in Figures 12 and 13 , which indeed show less change with increasing order. S8 P7 was chosen for the calculations.

An estimate of the error Involved in neglecting the absorption probability for neutrons leaving the core was made by putting anit outgoing source at the core radius at several different energles and allowing the code to calculate the flux returning to the core at all lower energies: By multiplying this flux by the first filght capture probability, an estimate of the number of absorptions was made. For the energies tested, the probability of capture for extefing neutrons was less than $1.1 \%$ of that for incident neutrons. This is to be expected since the hydrogen of the polyethylene is a forward scatterer; and neutrons elther leak from the spectrometer or, if they do return, are slowed down below the cadmium cut-off. 


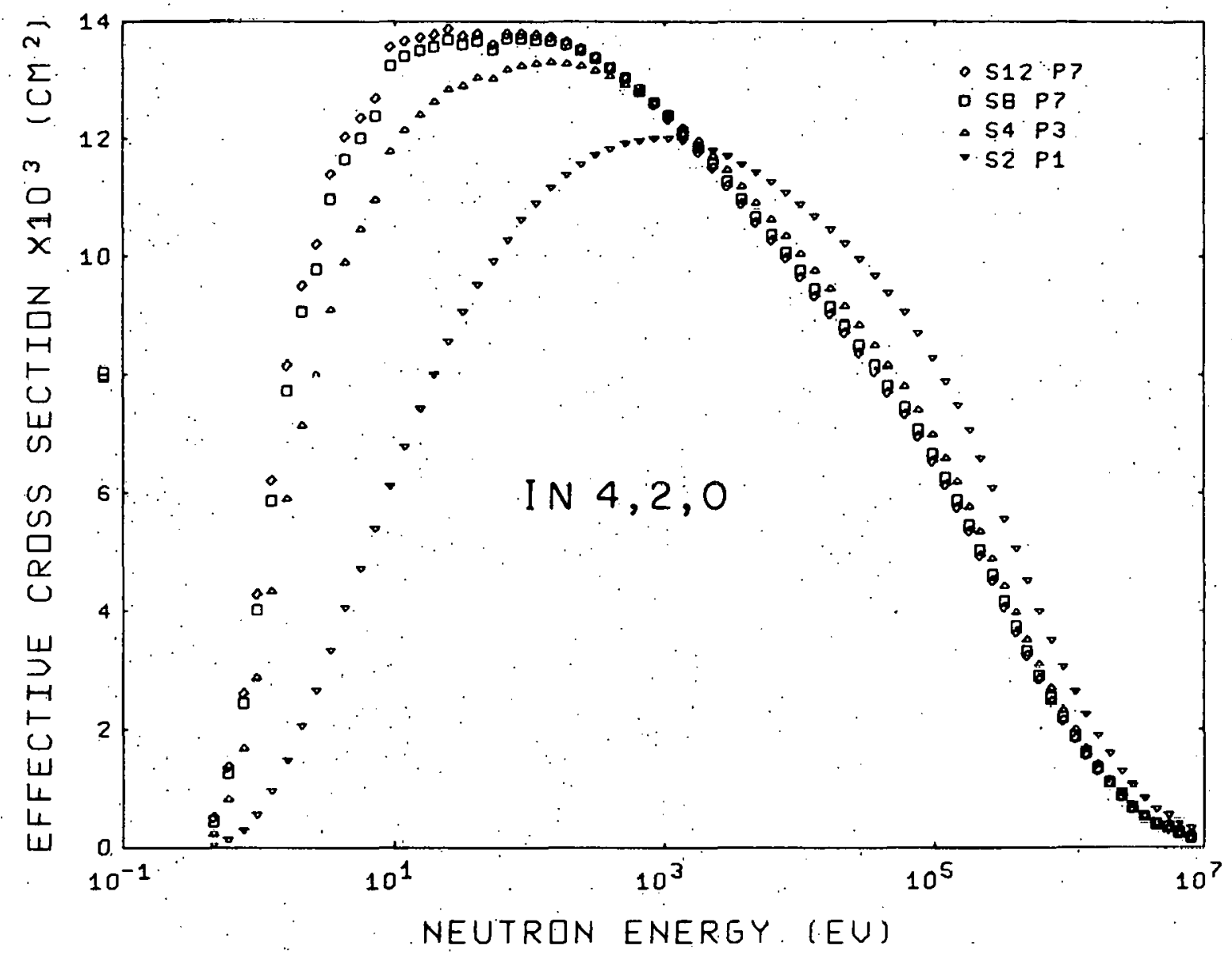

XBL7O12-4190

FIGURE 11. $\sigma_{L S}(E)$ for In $4,2,0$ for Several Combinations of Quadrature and Scattering-Kernei order 
51

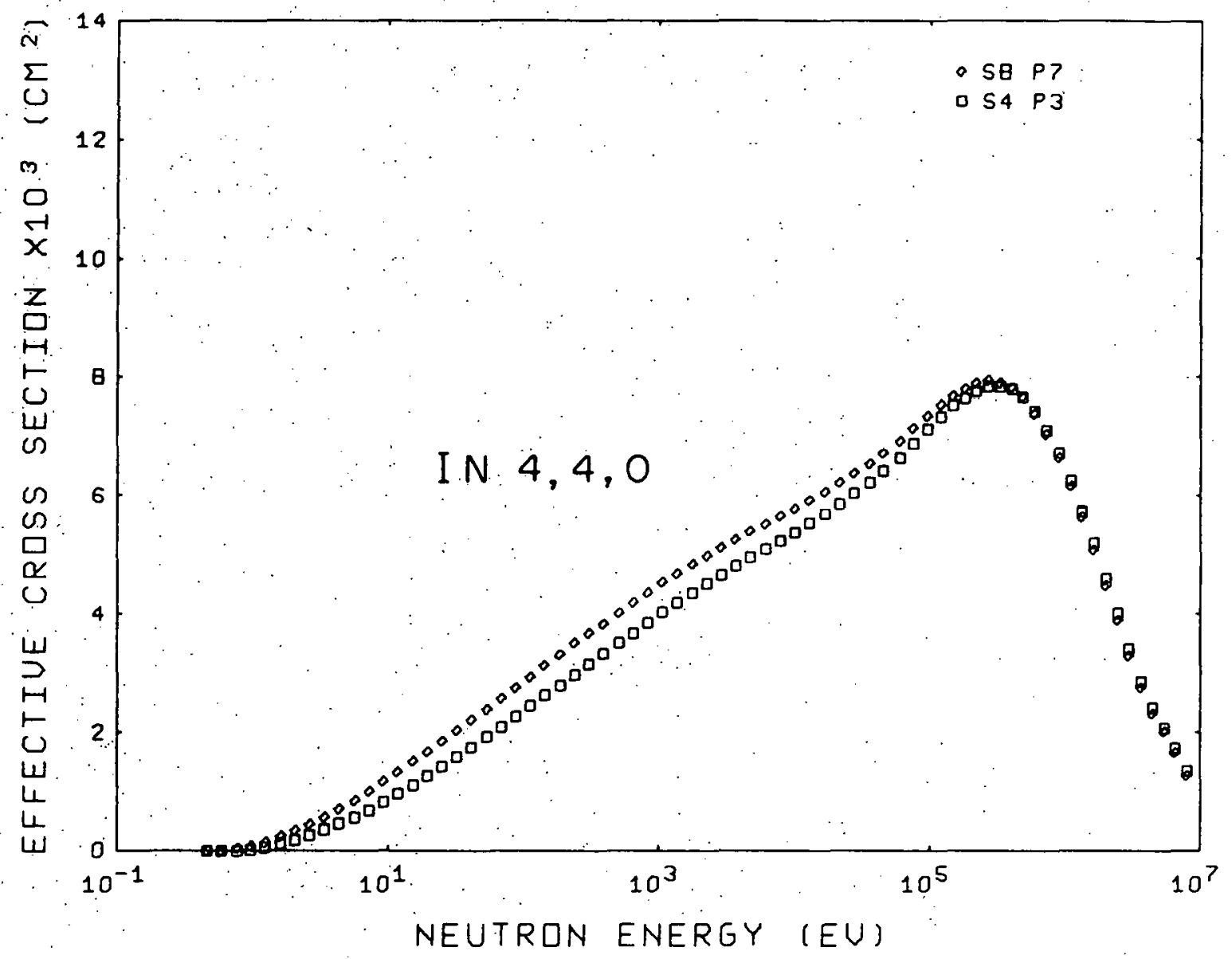

$X B L 7012-4191$

FIGURE 12. $\sigma_{\text {LS }}$ (E) for In 4,4,0 for Two Combinations of Quadrature, and Scattering-Kernel order 


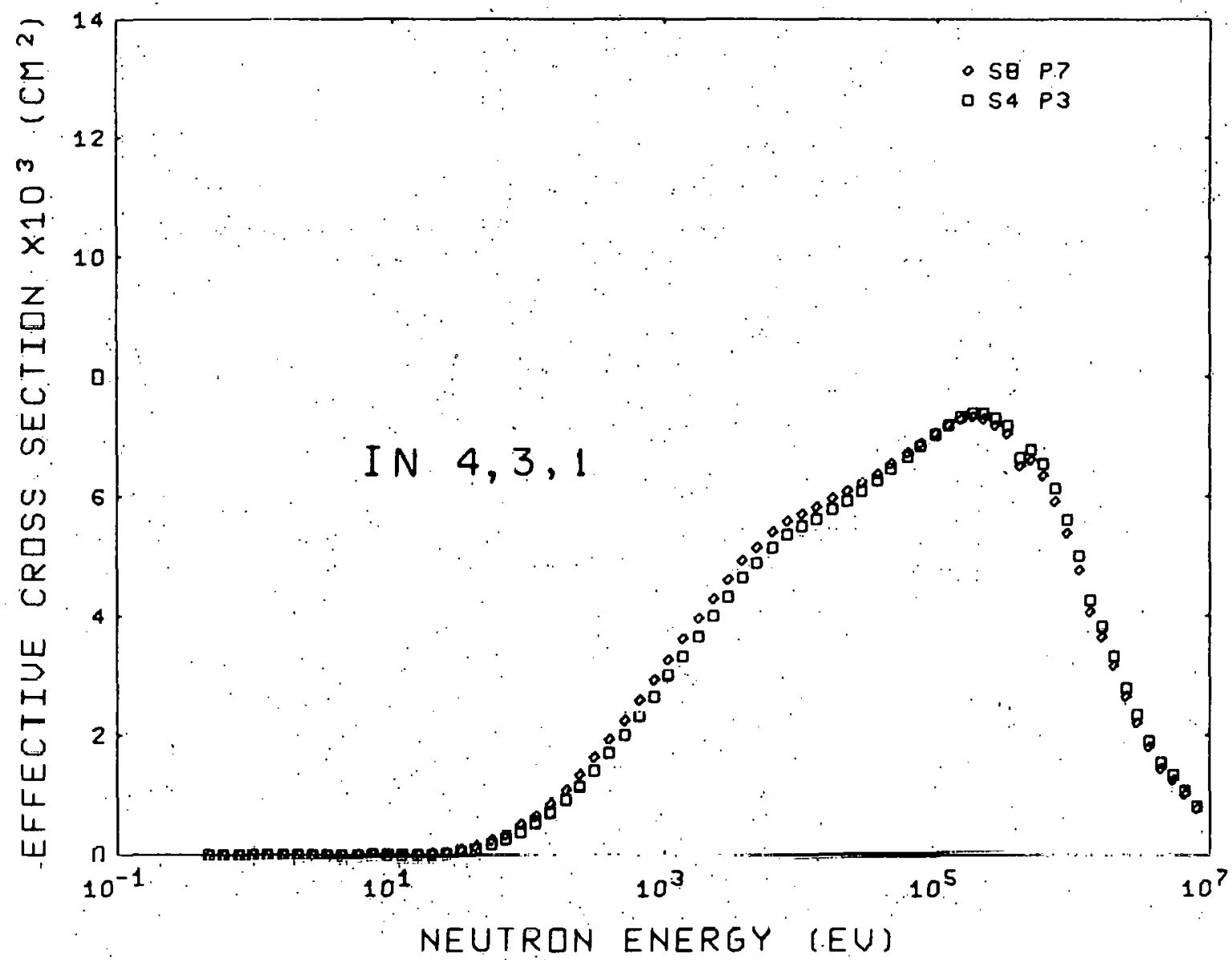

$X B L 7012-4192$

FIGURE 13: $\dot{\sigma}_{\text {IS }}$ (E) for In $4,3,1$ for Two Combinations of Quadrature and scattoring-Kernel order 


\section{E. Results}

Calculations were performed for bare polyethylene for diameters varying from 2 inches to 10 inches; the results are given in Figures 14 and 15 . It can be seen that response for the smaller spheres is extremely broad. several decades at half maximum. As the diameter is increased, the response first flattens, with the second derivative with respect to lethargy. on the low energy side of the peak becoming less negative until at 4" the second derivative is almost zero. Further increase in diameter improves the resolution, and the second derivative becomes positive. The unevenness of $\sigma_{L S}(E)$ caused by the resonance structure of the core material for the $2^{\prime \prime}$. sphere becomes smoothed as polyethylene is added until, it once again exhibits structure at 2 MeV due to resonances in the carbon crosis section. It can also be seen that responses of high resolution are imposstble to attain in the kev region. In Figures 16-19 the results of calculations for various thicknesses of boron carbide powder are given. As with the modified Bonner spheres (Figure 4) the peak response is lowered in value and shifted to higher energy. The bare sphere response does not however form an envelope for the spheres, whth boron added as in Metha's curves. This is because the calculations here did not neglect the small but finte moderating abllity of boron carbide (i.e., boron is not a pure absorber at high energy). The 


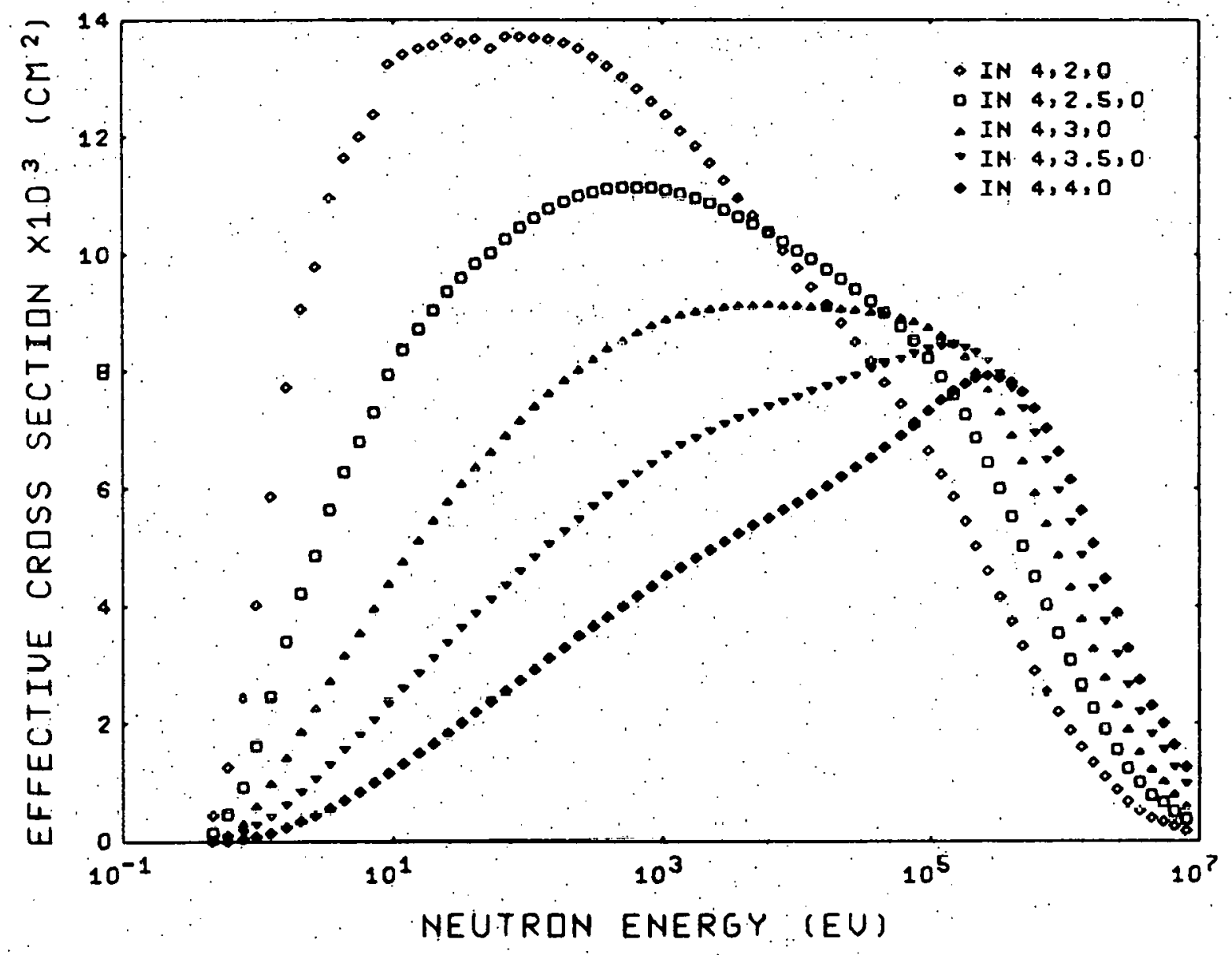

X B L.7012-4184

FIGURE 14. $\sigma_{\text {LS }}(E)$ for 4 mm Diameter Indium Core with. Polyethylene Diameters Varying from $2-1$. Inchics 


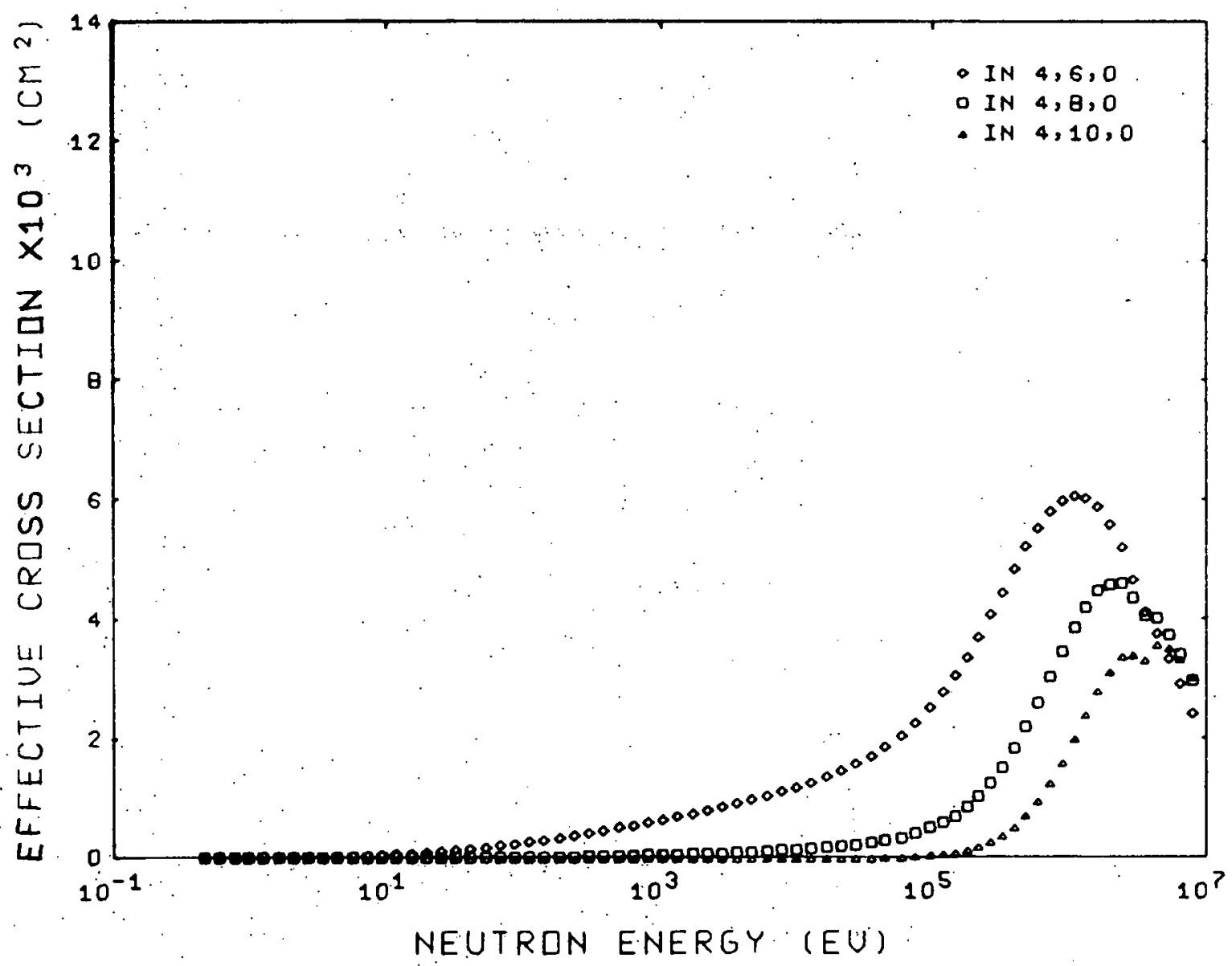

XBL7012-4195

FIGURE 15. $\sigma_{\text {LS }}(E)$ for $4 \mathrm{~mm}$ Diameter Indium Core with Polyethylene Diameters Varying from 6-10 Inches 


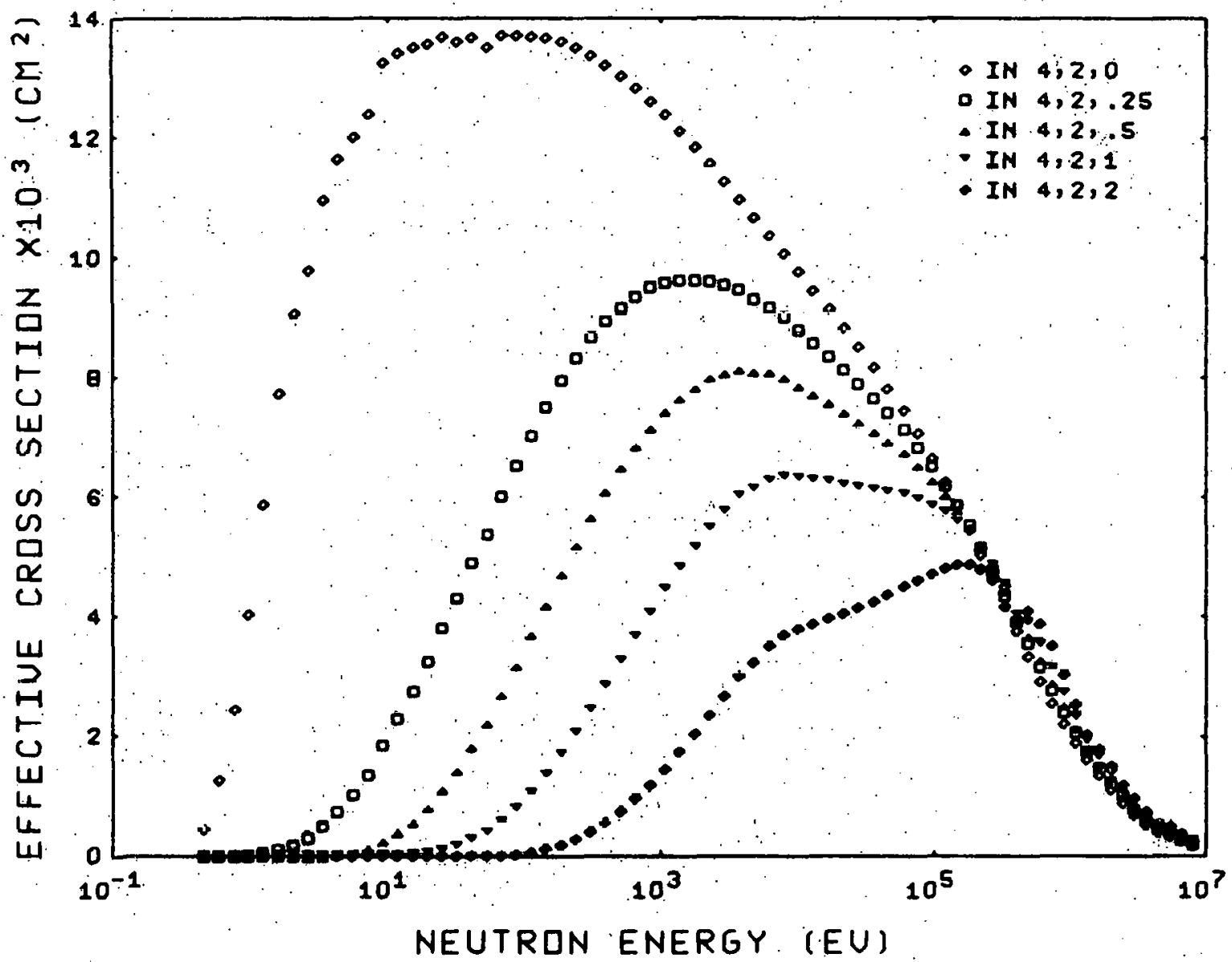

YBL, $7013-7360$

FIGURE 16. $\sigma_{\text {LS }}($ E) for 4 mm Diameter Indium Core,

2 Inch Polyethylene niameter, and Boron-

Carbide Powder Thicknesses Varyıng from

0-2 Inches : $.25, .5,1,2$ Inches Correspond to $.55, .97 ; 1.60,3.74 \mathrm{gB} / \mathrm{cm}^{2}$. 


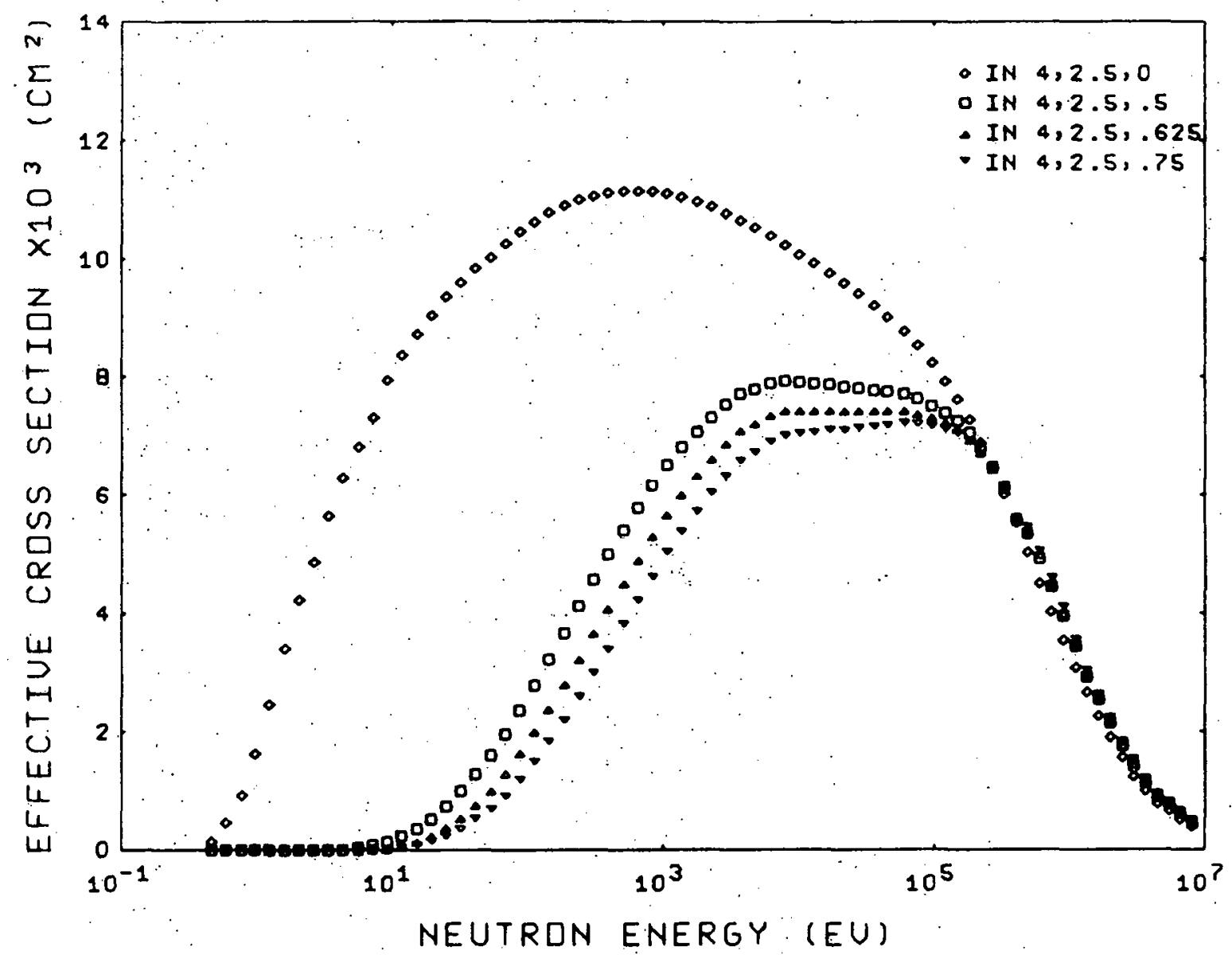

XBL $7012-4186$

FIGURE 17. $\sigma_{\mathrm{LS}}(\mathrm{E})$ for 4 mm Diameter Indium Core, 2.5 Inch Polyethylene Diameter, and BoronCarbide Powder Thicknesses Varying from $0-.75$ Inches. $.5, .625, .75$ Inches Correspond to $1.04,1.22,1.40 \mathrm{gB} / \mathrm{cm}^{2}$. 


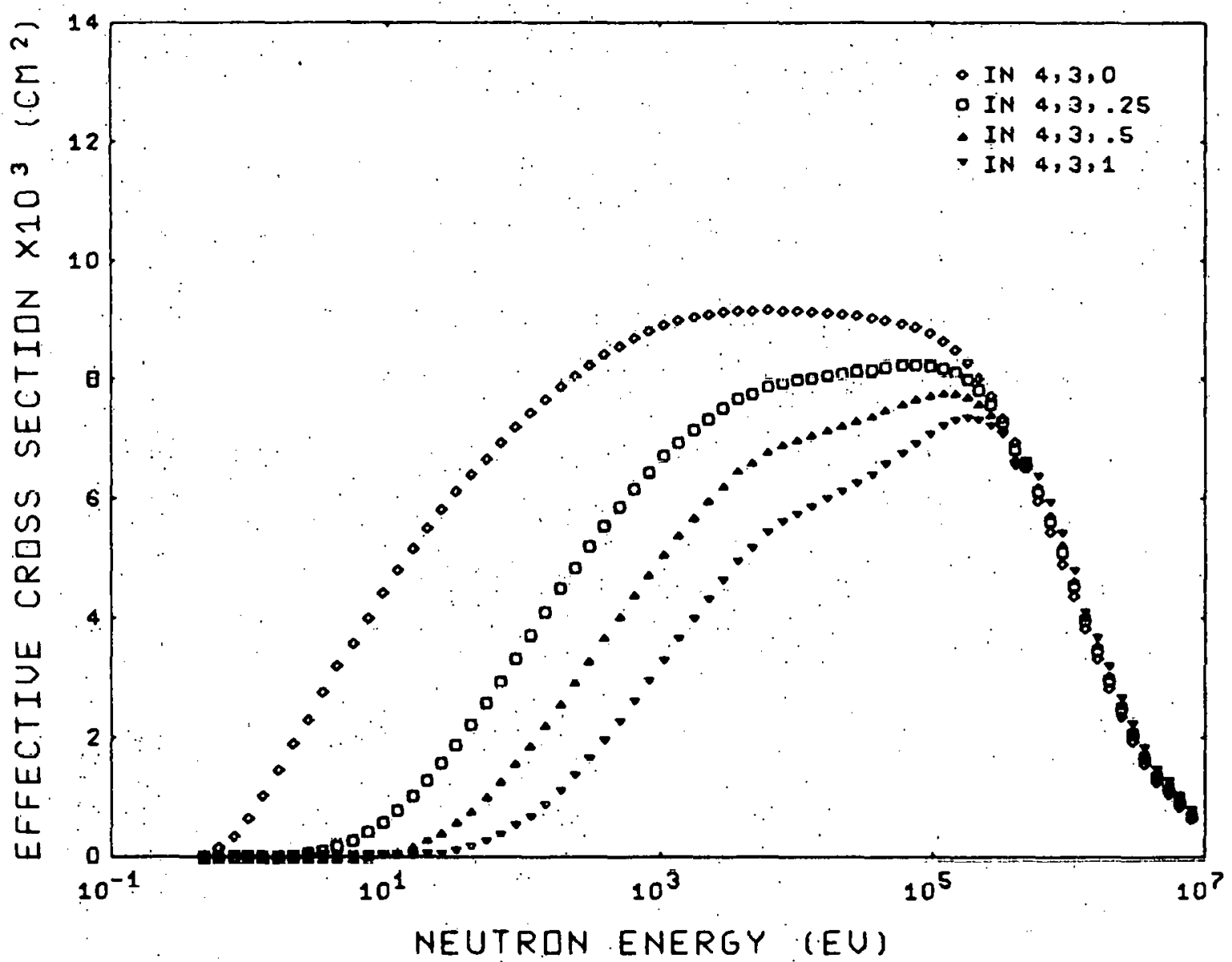

X8L7012-4187

FİGURE 18. $\sigma_{L S}(E)$ for 4 mm Dlameter Indium Core.

2 Inch Polyethylene Diameter, and Boron-

Caibldu Thlckncoscs Varying from $0-1$ Inches

$.25, .5,1$ Inches Correspond to $.60,1.07$,

$1.67 \mathrm{gB} / \mathrm{cm}^{2}$. 


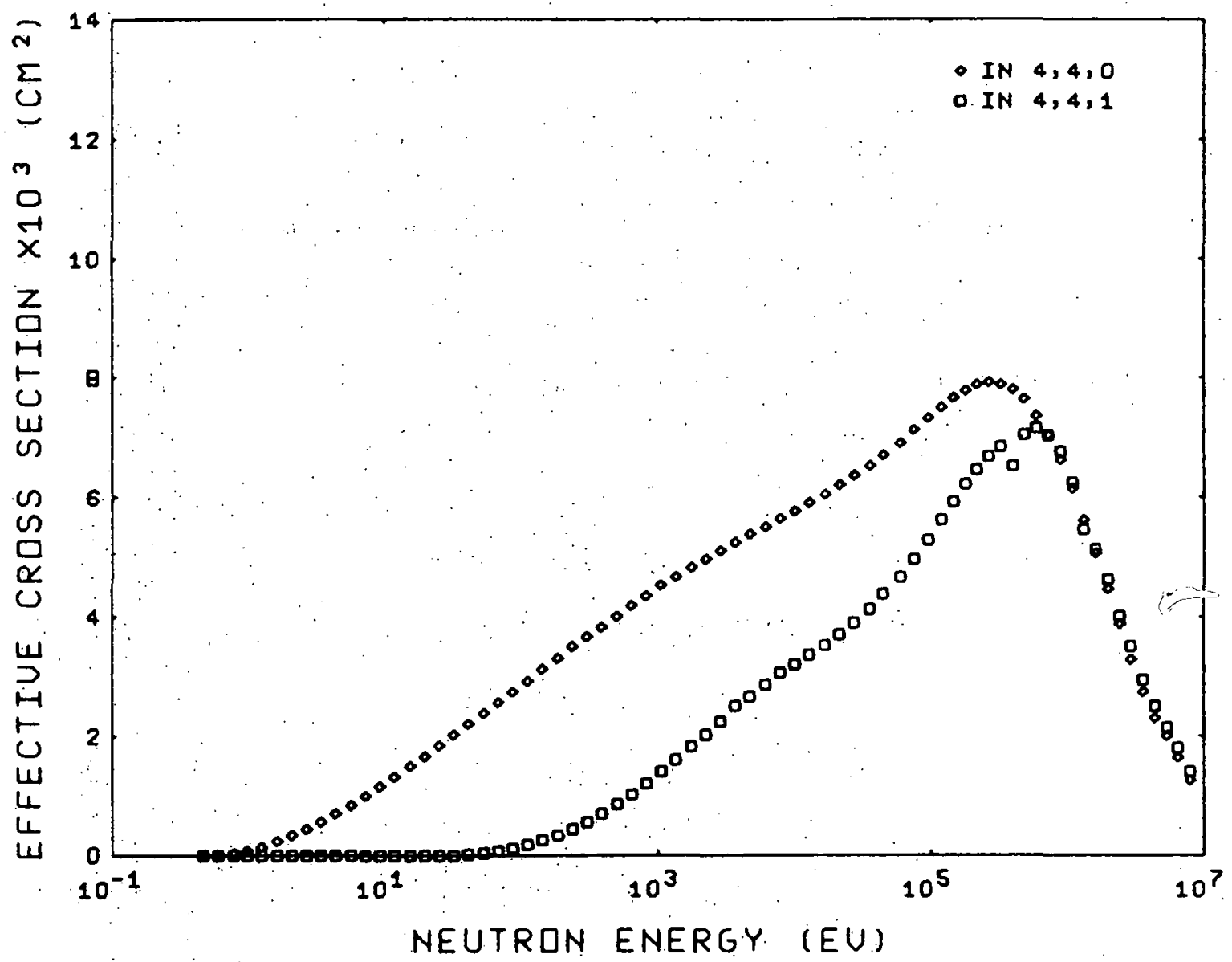

XBL $7012-4188$

FIGURE 19. $\sigma_{L S}(E)$ for 4 mm Diameter Indium Core 4 Inch Polyethylene Diameter, and BoronCarbide Powder Thicknesses of 0 and 1 Inch. 1 Inch Corresponds to $1.96 \mathrm{gB} / \mathrm{cm}^{2}$. 
minimum (most evident in Figure. 19) that occurs at about 500 keV for thicker boron shells is caused by a resonance in the boron cross section which scatters neutrons away from the sphere, decreasing the probability of penetrating to the core. The peak posttion for a given $\mathrm{CH}_{2}$ diameter is not a simple function of the boron thickness, because of the complicated combination of scattering and absorption in the boron. Small changes in the thickness cause large changoo in peak shape, making ic difficult to place the maximum response where desired (see Figure 17).

Figure 20 shows $F_{g}\left(R_{L S}, \mu\right)$ for In $4,2,0$. Of interest is the peaking at $\mu=-1$ and the lessening of this peak as energy increases. The results for a $4 \mathrm{~mm}$ indium core are summarized in Figure: 21 , where response maximum value, energy position, and resolution (full width at half maximum) are represented. From this figure; a set of spectrometers can be chosen according to the needs of the situation. One may, for instance, for a given peak position want to trade resolution for peak value or vice vorsa. This figure is intended to be used directiy or to serve as a basis for interpolation for construction of spheres. For constructions using a form of boron other than $B_{4} \mathrm{C}$ powder, the thicknesses are given in $\mathrm{gB} / \mathrm{cm}^{2}$ fn the figure captions.

Responses werc also calculated for a 4 mm gold absorber, and the results are given in Figures 22 and 23 . The shapes are similar to those of indium, as expected, since the 


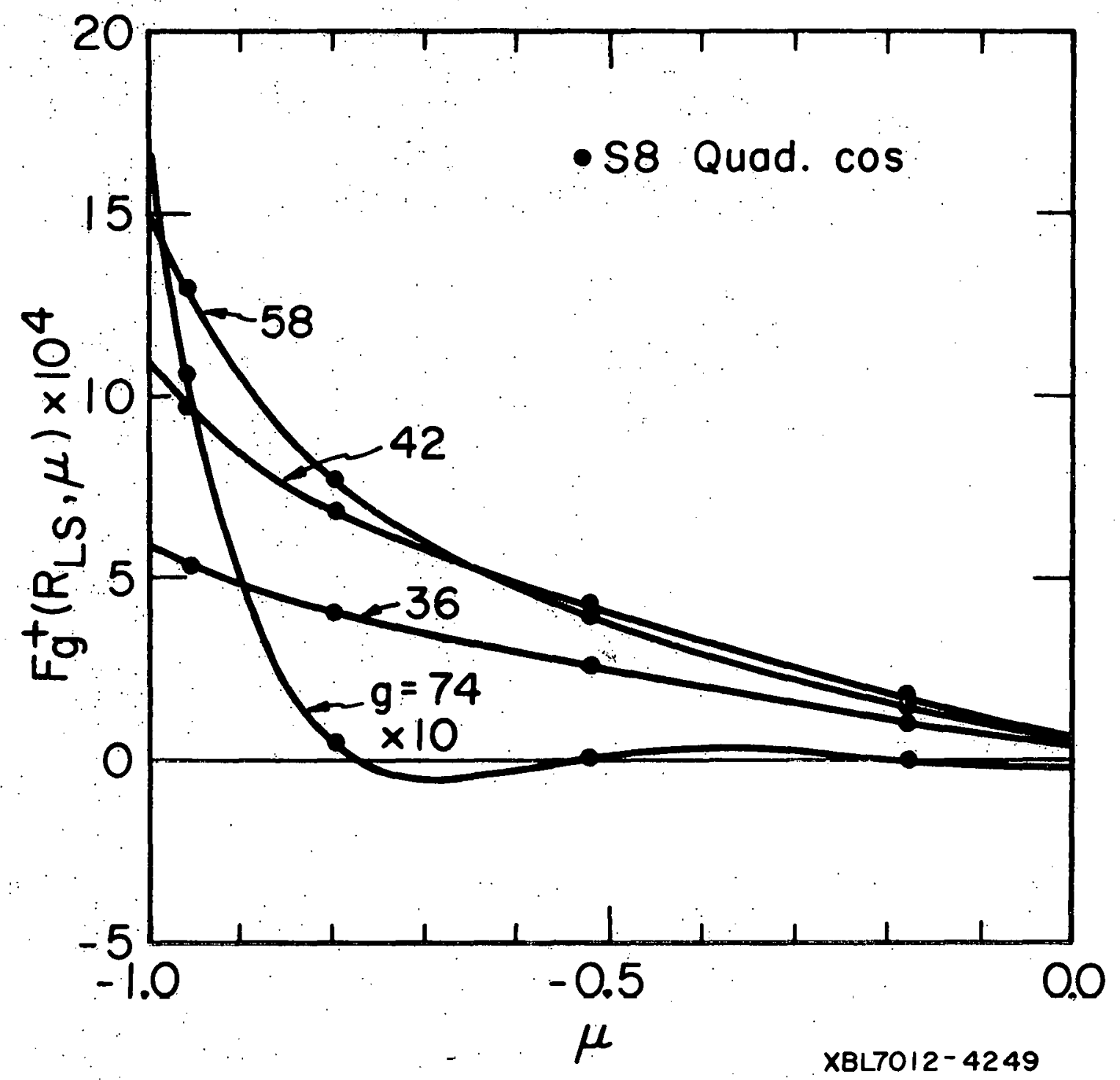

FIGURE 20. $F_{g}^{+}\left(R_{L S}, \mu\right)$ for In $4,2,0$ for $g=36,42,58$ and 74 (groups numbered as in Table 3) 


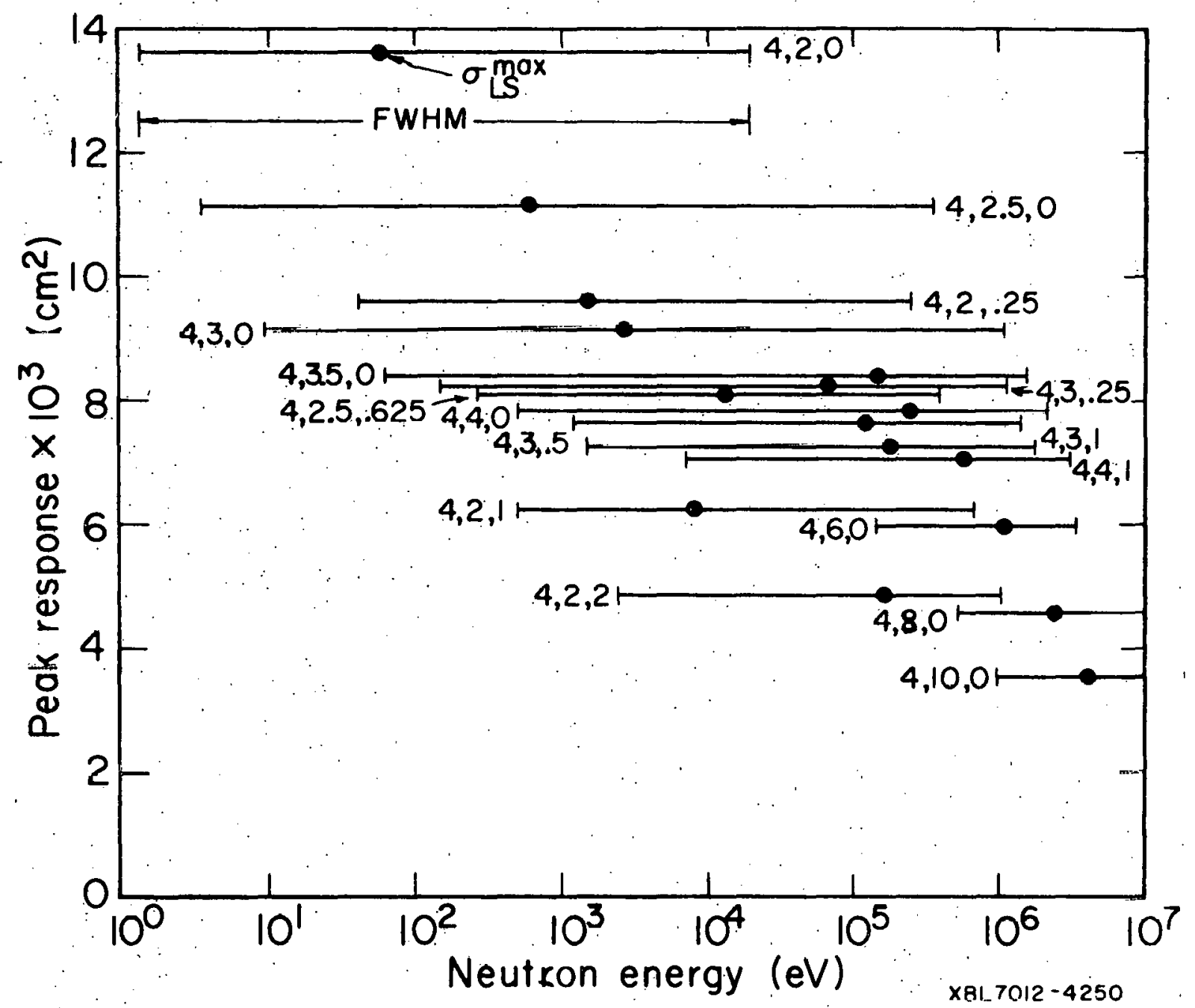

FIGURE 21. Peak Response Position and Resolution. for LS's with $4 \mathrm{~mm}$ Diameter Indium Cores as a Function of Energy 


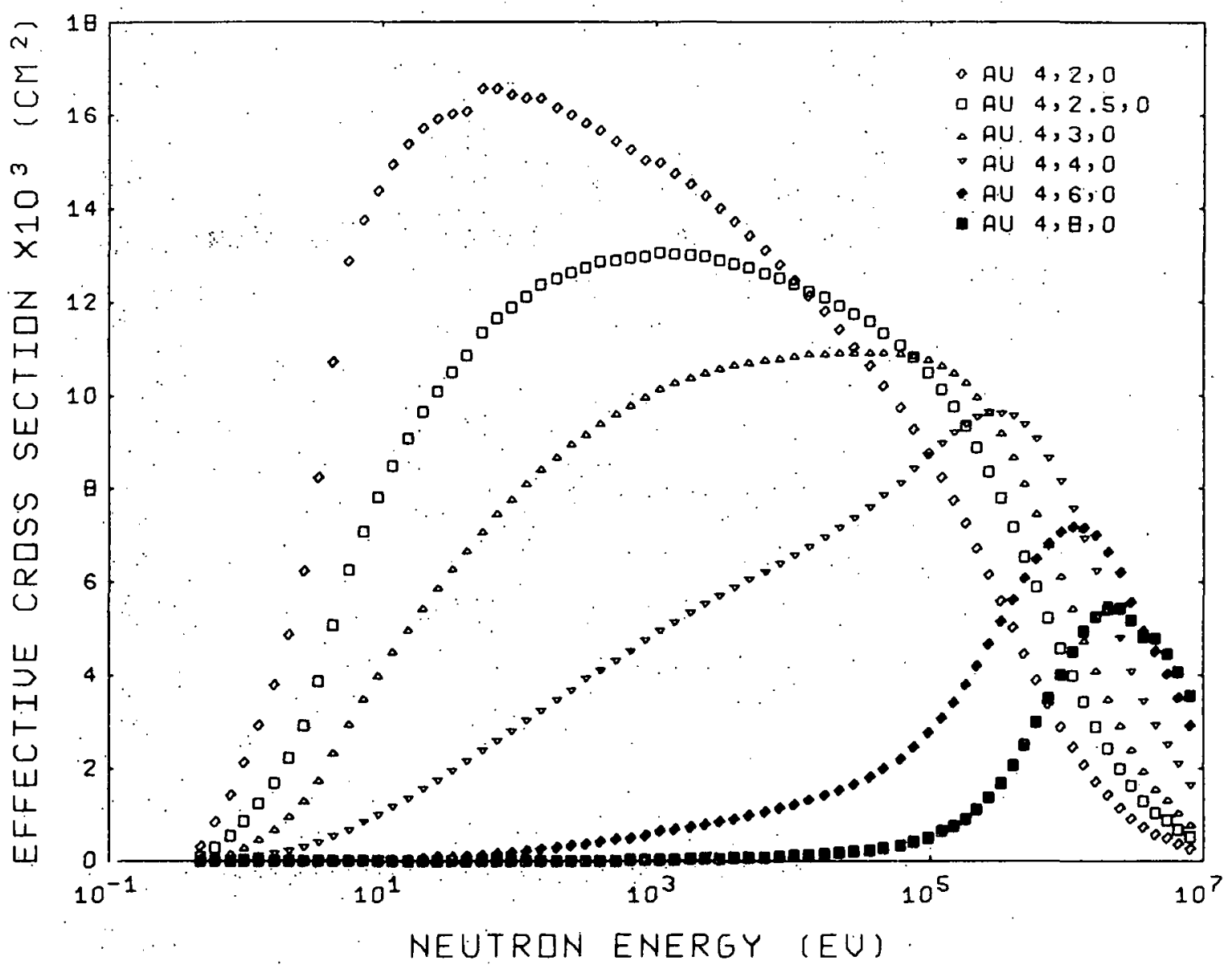

$X B L 7012-4189$

FIGURE 22. $\sigma_{\text {LS }}(E)$ for $4 \mathrm{~mm}$ Diameter Gold Core and Polyethylene Dímeters Varying from 2-8 Inches 


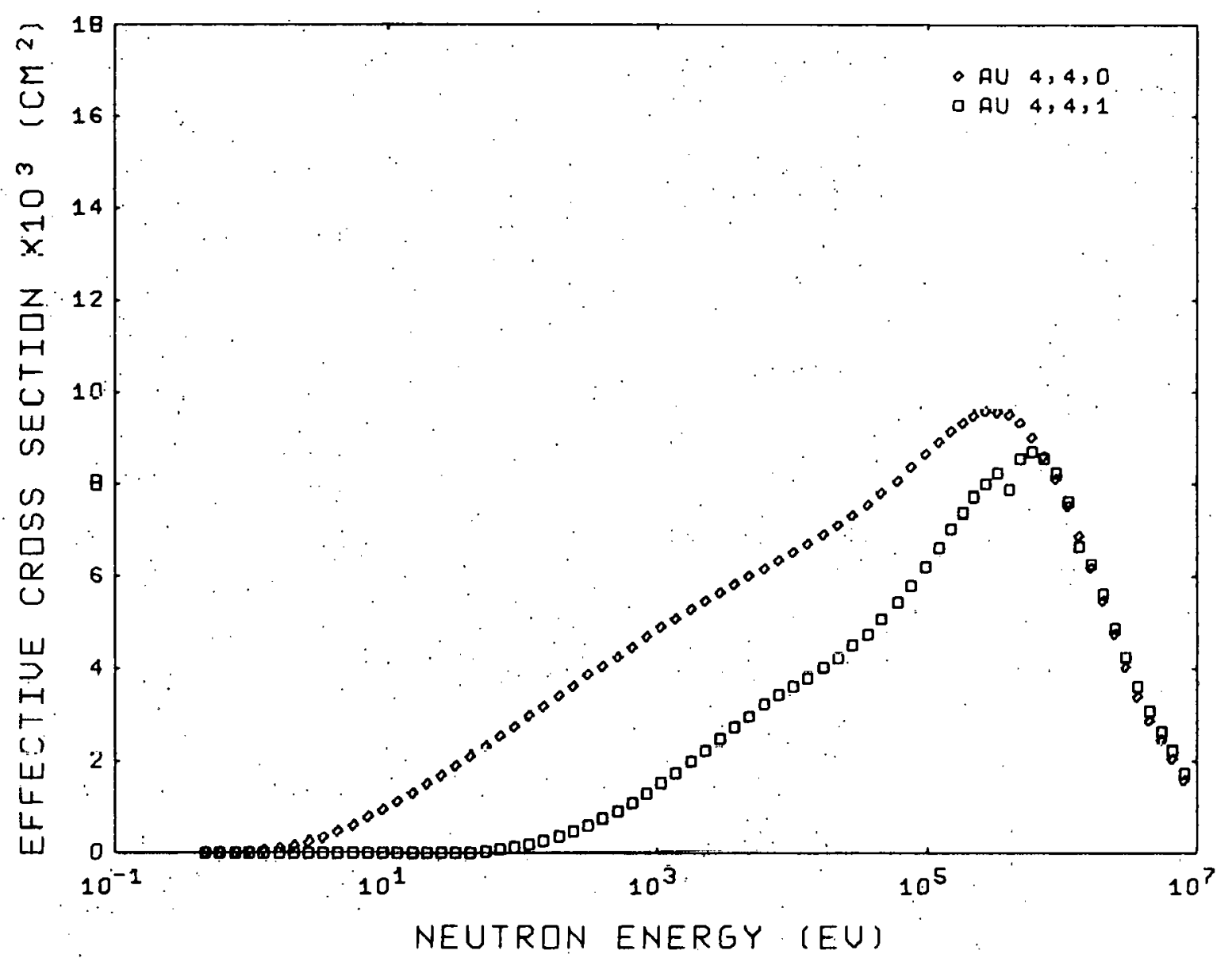

$X B L 7012-4194$

FIGURE 23. $\sigma_{\text {LS }}(E)$ for 4 mm Diameter Gold core,

4 Inch Polyethylene Diameter and Boron-

Carbide Powder Thicknesses of 0 and 1

Inch. I Inch corresponds to $1: 96 \mathrm{gB} / \mathrm{cm}^{2}$. 
major contribution to the capture is the $4.91 \mathrm{eV}$ resonance, whlch tis only $3.4 \mathrm{eV}$ from the principal indium resonance. The responses are about $20 \%$ higher for all energies because, as mentioned above, all captures lead to activation compared to $78.9 \%$. for indium. Indium has the advantage of reaching saturation more quickly (half-11fe of 54 min compared to 2.7 d for gold); hence, for a given flux, better counting statiotics can be obtainedin a short time. Gold is superior in that it need not be counted immediately after Irradiation. Its cross sections have also been evaluated in more detail and are therefore more reliable.

$\sigma_{\text {LS }}(E)$ was also determined for two spheres with a $4 \mathrm{~mm}$ core of manganese, which has its first resonance at $337 \mathrm{eV}$ (results 1n Fig. 24). The response for the larger sphere. (Mn 4,4,0) was similar to that of the indium core and shifted only slightly to higher energy for the 2.5 inch diameter sphere. Because the resonances of ${ }^{55} \mathrm{Mn}$ are largely. scattering (the resonance integral as defined in Eq. I. I is only 15 barns, of which 5.7 barns is $1 / v$ ), and the $1 / v$ variation from $337 \mathrm{eV}$ down to the cadmium cut-off, a large portion of the absorption takes place at low energy (below the 337 eV resonance); hence, the small shifts in response compared to In: In view of the above and the fact that the responses for a manganese core are almost an order of magnitude lower, it would be better 


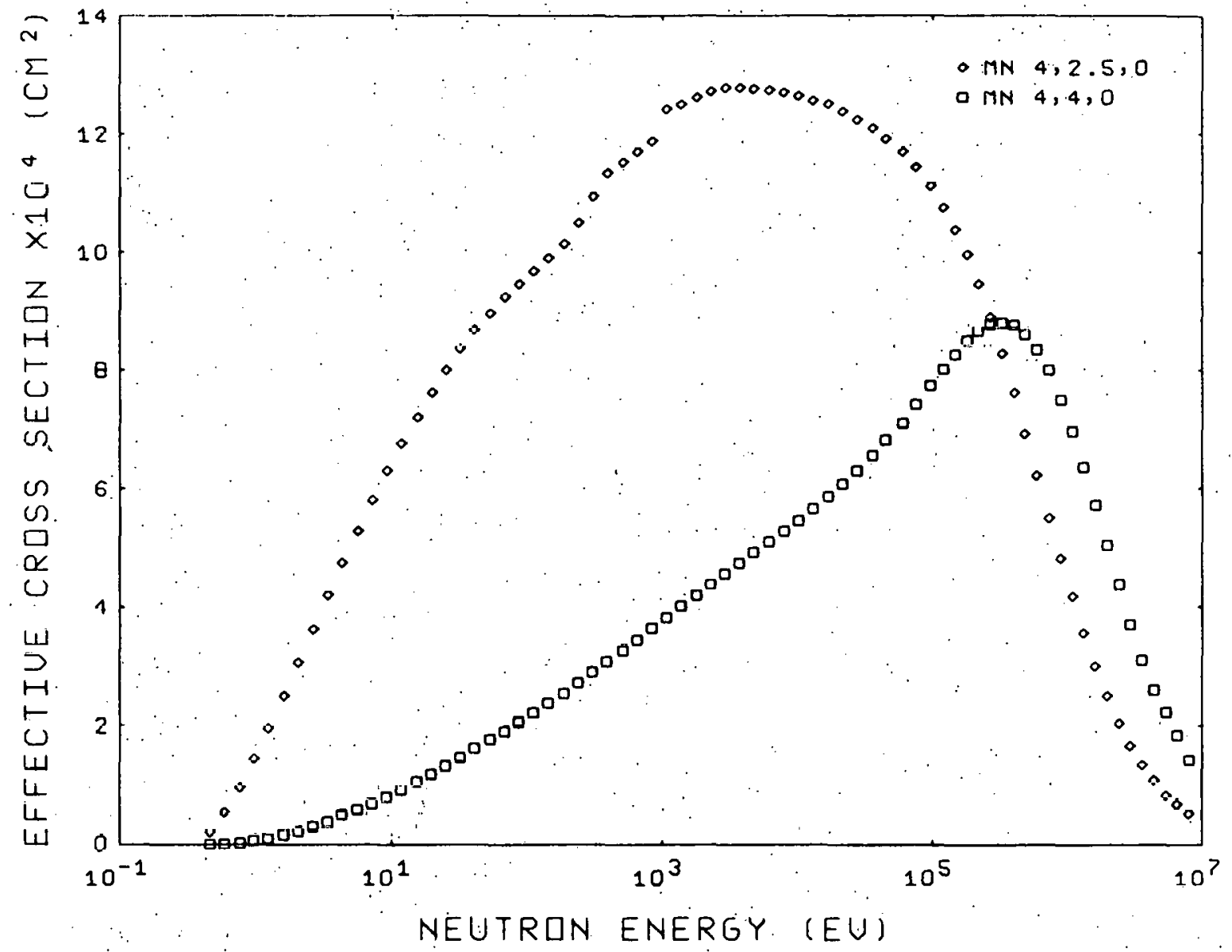

X8L $7012-4193$

FIGURE 24. $\sigma_{\text {LS }}(E)$ for 4 mm Diameter Manganese Core and Polyethylene Diameters 2.5 and 4 Inches 
to choose a polyethylene-boron configuration with a gold or indium core and the same shape. 


\section{EXPERIMENT}

A. Apparatus and Experimental Results

In this section we summarize the work of S. Binney, Bi70 who performed an experiment to calibrate and check the calculations of four leakage spectrometers ( In $4,2,0$;

In $4,2,1 ;$ In $4,4,0$; and In $4,3,1$ ). Results of the experiment are given, and in the section that follows they are compared to the theoretical results of Chapter II.

The calibration of the Leakage spectrometers (LS's) was carried out with neutrons from the ${ }^{9} B e(\gamma, n)$ reaction, whose threshold is 1.665 MeV. With the proper choice of activated isotopes - to produce the gamas - neutrons with keV energies were produced. The isotopes that were activated for gamma emissinn aro listod in Table 5 , sluwlug the neutron energies obtained. Salts of the material to be aclivated were disolved in water and pumped through a container next to the core of the UC TRIGA Mark III research reactor. The container was part of a continuous flow loop. Af the other end of the loop, the activated solution flowed between concentric spherical copper shells (see Figure 25), 18 and 20 inches in diameter respectively. The liquid between the shells constituted the gamma ray source, producing neutrons through the irradiation of a beryllium shell Inside the Inner copper shell. The beryliium target "sphere" Was constructed by epoxying many small flat beryllium sheets (1/16"thick) to the Inside of the copper 
TABLE 5

NEUTRON SOURCE DATA

\begin{tabular}{|c|c|c|c|c|c|c|}
\hline Isotcpe & $\begin{array}{l}\text { Compound } \\
\text { for Solution }\end{array}$ & $i$ & $\begin{array}{l}\text { Gamma } \\
\text { Energy }\end{array}$ & $\bar{\sigma}_{\gamma_{n_{L}}}{ }^{m b}$ & $\begin{array}{l}\text { Gamma } \\
\text { Fraction } \\
F_{i}\end{array}$ & $\begin{array}{l}\text { Neutron } \\
\text { Energy } \\
\text { E }_{\text {Si }}\end{array}$ \\
\hline$A 1-\approx 8$ & $\mathrm{Al}_{2}\left(\mathrm{SO}_{4}\right)_{3}$ & & $1.78 \mathrm{MeV}$ & 0.88 & 1.00 & $102 \mathrm{ke} \mathrm{V}$ \\
\hline$M n-56$ & $\mathrm{Mn}\left(\mathrm{SO}_{4}\right)$ & 1 & $1.811 \mathrm{MeV}$ & 0.74 & .291 & $130 \mathrm{keV}$ \\
\hline & 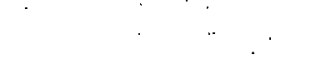 & 2 & $2.110 \mathrm{MeV}$ & 0.41 & .15 & $3.95 \mathrm{keV}$ \\
\hline In-116 & $\mathrm{In}_{2}\left(\mathrm{SO}_{4}\right)_{3}$ & 1 & $1.753 \mathrm{MeV}$ & 1.03 & .015 & $78 \mathrm{keV}$ \\
\hline & . & 2 & $2.110 \mathrm{MeV}$ & 0.41 & .195 & $396 \mathrm{ke} \cdot \mathrm{V}$ \\
\hline$S b-124 *$ & & & $1.69 .2 \mathrm{MeV}$ & 1.262 & .46 & $24 \mathrm{keV}$ \\
\hline
\end{tabular}

* A solid source was used in this case. 
70

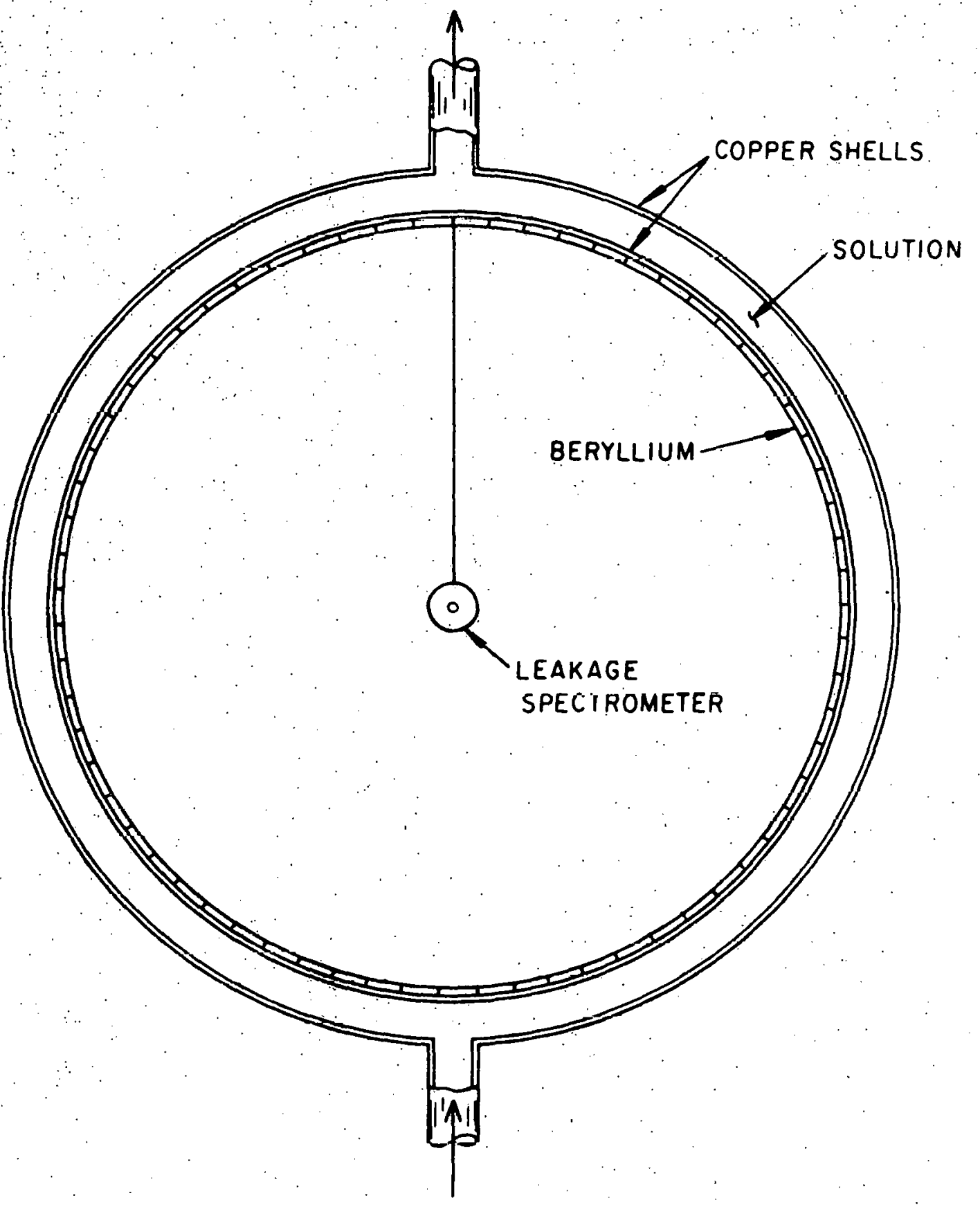

XML $7012-7356$

FIGURE 25. Spectrometer Calibration Assembly. 
sphere. The neutron flux near the center of the assembly, where the spectrometers were placed, was nearly isotropic and strongly peaked at the energy determined by the particular gamma ray intitiating the $(\gamma, n)$ reaction. The remalnder of the loop consisted of a pump to keep the liquid circulating, an expansion tank to permit monitoring of the liquid and allow for any thermal expanston, and a tank to allow dralnage. The relative neutron source strength for different solutions was monitored by a manganese ( $\mathrm{MnSO}_{4}$ ) bath through which a section of the loop consisting of a pipe surrounded by a beryl11um cylinder was passed. Neutrons from the Irradiated cylinder were captured by the manganese and the activity was measured. For an absolute measurement of the source strength, a $1 / 2$ in. diameter hollow cadmium sphere packed with lodine was activated and counted. A diagram of the entire flow system showing the various components is shown in Figure 26. Surrounding the spill retention tank was concrete personnel shielding, since the activity contained in the loop approached $500 \mathrm{Ci}$ at times.

In addition to the flow loop sources a solid $10 \mathrm{Ci}$ $\mathrm{Sb}-\mathrm{Be}$ source producing 24 keV neutrons was used. The values of the saturated activity A sat of the indium cores of the four LS's and the cadmium covered iodine for the four sources are given in Table 6 : 


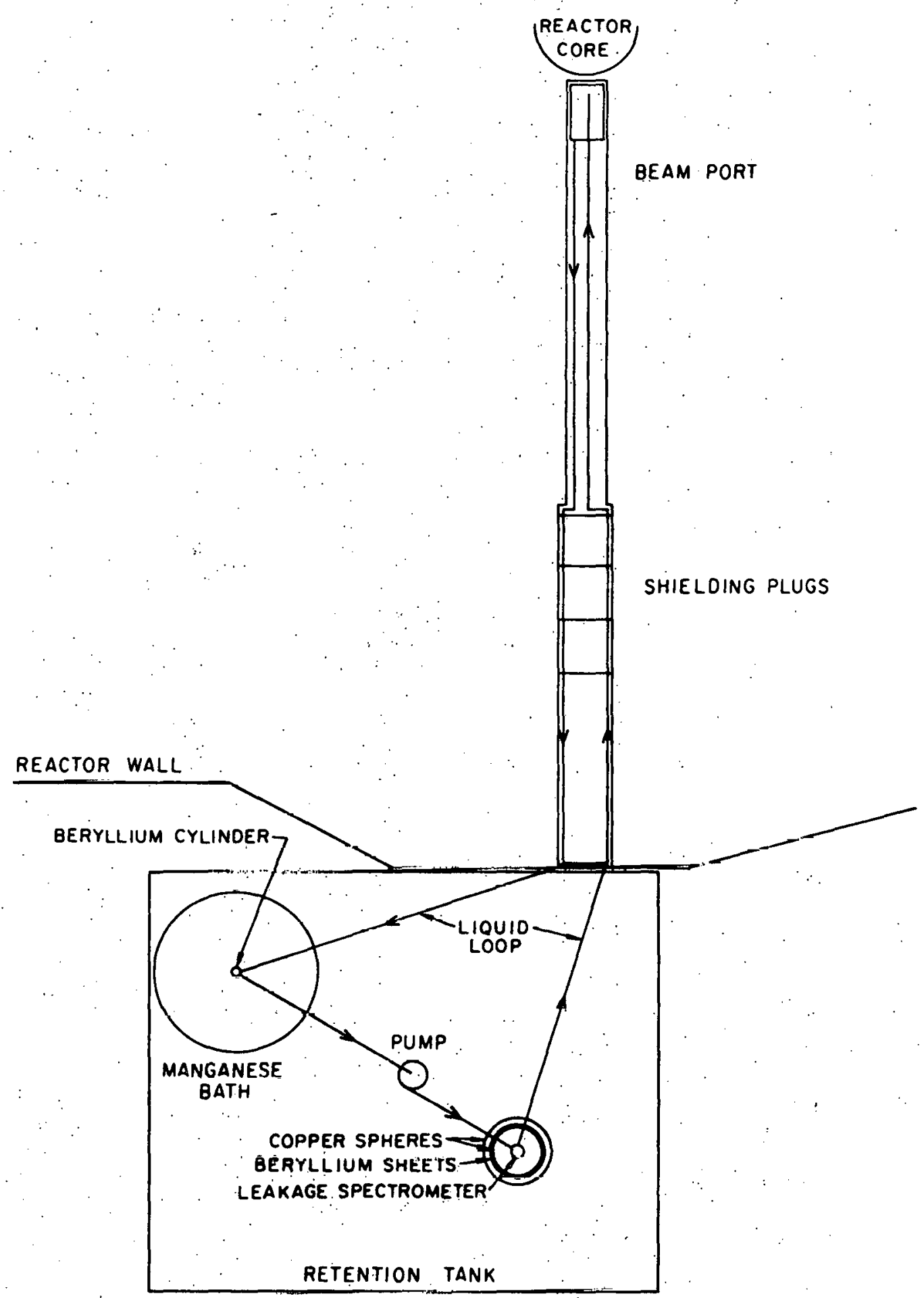


TABLE 6

SATURATED ACTIVITY

\begin{tabular}{|c|c|c|c|}
\hline Source & Detector & $\mathrm{A}_{\text {sat }}, \mathrm{dps}$ & $\Delta \mathrm{A}_{\text {sat }}, \%$ \\
\hline \multirow[t]{5}{*}{$\mathrm{Mn}$} & $I-C d$ & 273 & 5.4 \\
\hline & In $4,2,0$ & 83.2 & 6.9 \\
\hline & In $4,4,0$ & 57.9 & 7.3 \\
\hline & In $4,2,1$ & 31.6 & 7.3 \\
\hline & In $4,3,1$ & 40.9 & 8.7 \\
\hline \multirow[t]{5}{*}{ In } & $I-C d$ & 67.9 & 5.1 \\
\hline & In $4,2,0$ & 24.2 & 5.1 \\
\hline & In $4,4,0$ & 15.0 & 6.6 \\
\hline & In $4,2,1$ & 10.5 & 5.9 \\
\hline & In $4,3,1$ & $=15.1$ & 5.4 \\
\hline \multirow[t]{5}{*}{ A1 } & $I-C d$ & 69.9 & 4.6 \\
\hline & In $4,2,0$ & 21.9 & 4.6 \\
\hline & In $4,4,0$ & 12.7 & 4.7 \\
\hline & In $4,3,1$ & 8.7 & 5.2 \\
\hline & $\operatorname{In} 4,3,1$ & 10.1 & 4.9 \\
\hline \multirow[t]{5}{*}{$\mathrm{S} \mathrm{b}$} & $I-C d$ & 11.1 & 5.5 \\
\hline & In $4,2,0$ & 6.3 & 5.8 \\
\hline & In $4,4,0$ & 4.7 & 5.9 \\
\hline & In $4,2,1$ & 3.6 & 8.0 \\
\hline & In $4,3,1$ & 4.0 & 6.1 \\
\hline
\end{tabular}




\section{B. Analysis of Experiment and Comparison of Results}

The neution source (Figure 25) was designed so that

the ingoing neutron flux at the surface of the spectrometer was nearly isotropic (a smaller Be shell would still have given rotational symmetry but as mentioned in section II.A translational invariance is required when the scalar flux is to be measured). The incldent spcctra for the four neutron source energies were calculated by ANISN, taking advantage of the spherical symmetry. In all cases the source was taken as isotropic within the Be shell and normalized to 1 neutron/sec spread over the group whirh contalned the source energy $\mathrm{E}_{S}$. The 99 group scheme in Table 2 with Input data from the DLC-9. set and the "fast" Iibrary for the GGC-4 code ${ }^{+}$was used. The shielding was approximated by a thick concrete shell concentric to the copper sphere.

For the present geometry Eq: II.I becomes

$$
A=4 \pi R_{L S}^{2} \int_{=4 e V}^{E} S \int_{-1}^{0} d \mu \mu F^{+}(E, \mu) F\left(R_{L S}, E, \mu\right)
$$

In particular, since the lincldent flux is lisotropic,

$$
P\left(R_{L S}, E ; \mu\right)=\phi\left(R_{L S}, E\right) / 2 \quad-1<\mu<0
$$

and :

†see footnote on page 41. 


$$
A=\int_{4 \mathrm{eV}}^{E} \mathrm{dE \phi}\left(R_{L S}, E\right) 2 \pi R_{L S}^{2} \int_{-1}^{0} d \mu \mu F^{+}(E, \mu)
$$

or

$$
A=N_{0} \int_{.4 \mathrm{eV}}^{E} \mathrm{dE} \hat{\phi}(E) \sigma_{L S}(E)
$$

where $\hat{\phi}$ is the scalar flux due to one neutron orfiginating in the beryllium at energy $E_{S}$, and $N_{0}$ is the total number of neutrons produced by the $(\gamma, n)$ reaction. This equation is correct for the aluminum-beryliium source. The indium and manganese solutions each produce neutrons wth contributions at two different energies and the integral has to be weighted with the fraction of neutrons produced at each energy. This fraction is determined by the product of the fractional occurrence ff the gamma ray causing the photoneutron reaction and the cross section $\sigma_{n \gamma}$ for the reaction (see Tabie 5). Thus the saturated activity

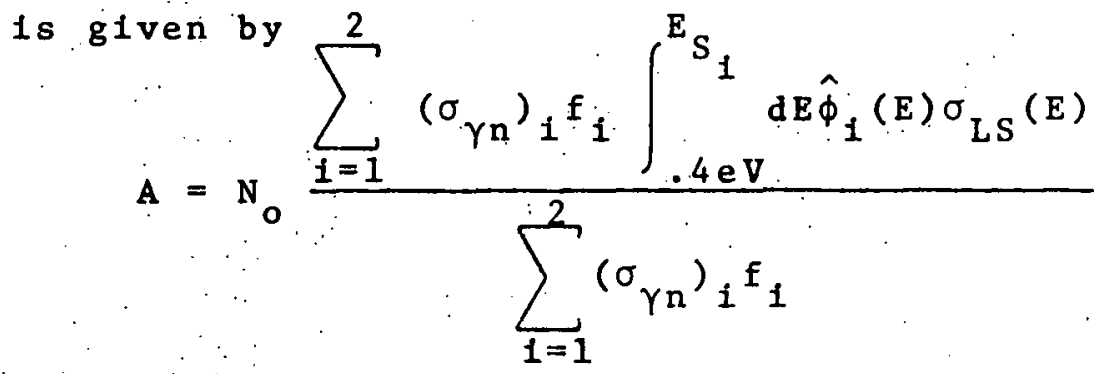

$$
\text { Results for } \hat{\phi}_{i}(E) \text { for } E_{S_{1}}=130 \mathrm{keV} \text { are given in }
$$

F1gure. 27 for two quadrature orders and concrete radi1. In this case a PN calculation denotes an $N+1$ order Gaus 


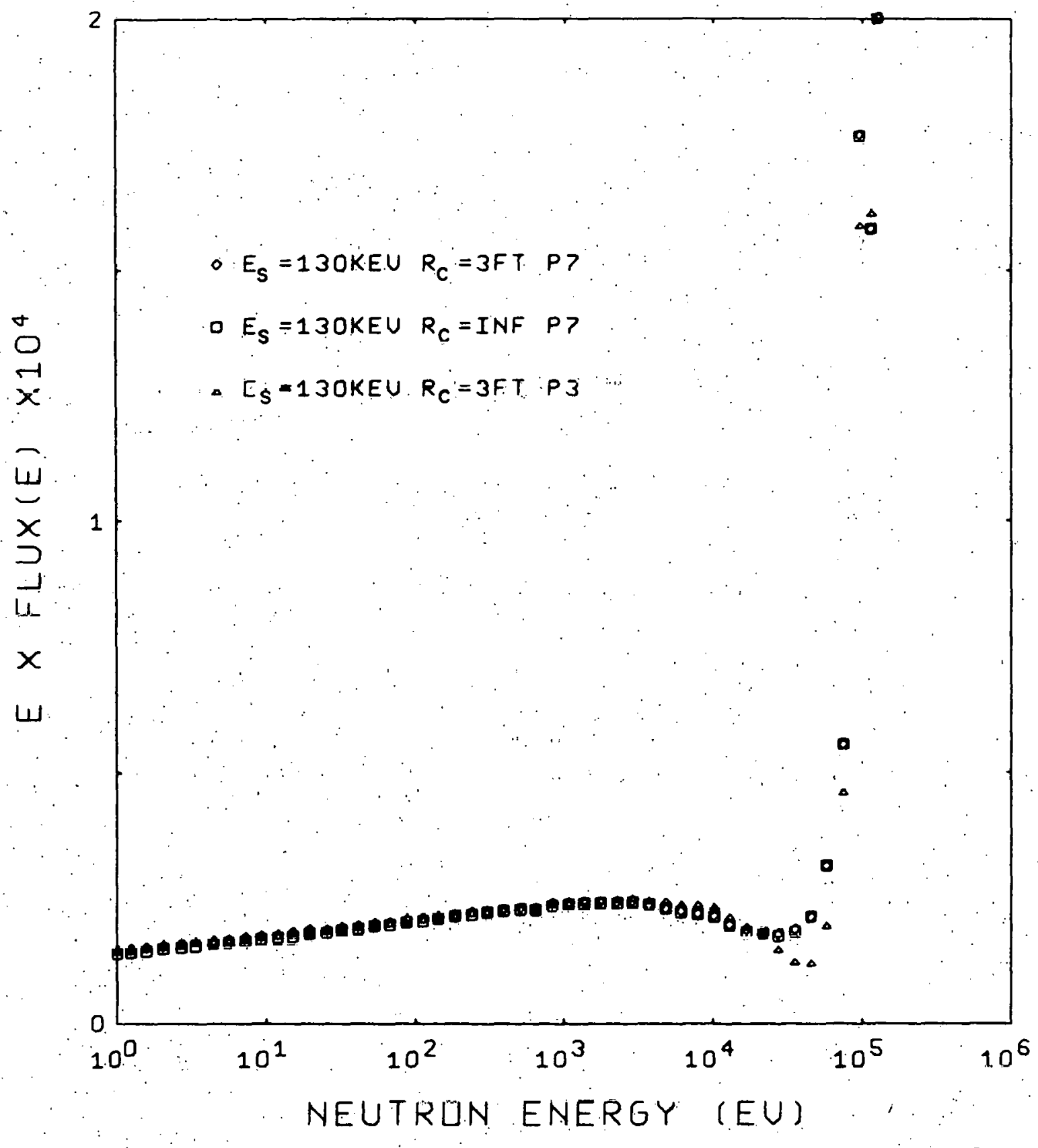

XBL $7012-4275$

FIGURE 27. Incident Scalar Flux for $E_{S}=130 \mathrm{keV}$ Comparison of three approximations. 
Legendre Quadrature with an $\mathrm{N}^{\text {th }}$ order expansion of the scattering kerne1. The concrete sheli. (2 ft. thick) located at a radius $R_{c}$ of $3 \mathrm{ft}$. is the lower bound on the distance between the copper shell and the concrete. Infinite $R_{c}$ corresponds to no concrete being present. The vartation for the two geometries is extremely small as can be seen in the: figures. The peak value (15.7) is identical, and the area under the curve for the case of no concrete is only $1 \%$ lower than that for a concrete radius of $3 \mathrm{ft}$.

Using P7 calculations and $R_{c}=3 \mathrm{ft}:$ the flux spectra were calculated for the four different source energies (see Figures 28 to 31 ): For the three $\gamma$-emitting solutions, $A / N_{0}$ for each of the four spectrometers tested was determined using III.1 or III.2. Values of A/No for the Sb-Be source (considered a point monoenergetic source at 9 inches) were obtained by dividing the value of $\sigma_{L S}$ from the curves in Chapter. II a't $24 \mathrm{keV}$ by $4 \pi \mathrm{R}^{2}$. As mentioned above, iodine was used as a flux monitor. A response $\sigma_{I}$ (E) was calculated In a manner similar to $\sigma_{\text {core }}(E)$. This was used together with III.1 or III.2 and the experimental values of A for lodine (Table 6) to determine $N_{0}$ for each of the four sources.

From $N_{0}$ the theoretical values of A were calculated. These are given in $\mathrm{Tab} l e \mathrm{~T}$ along with the experimental values. On the basig of the test calculations previously mentiosed, the errors in the theoretical value of $\mathrm{A} / \mathrm{N}$. 
78

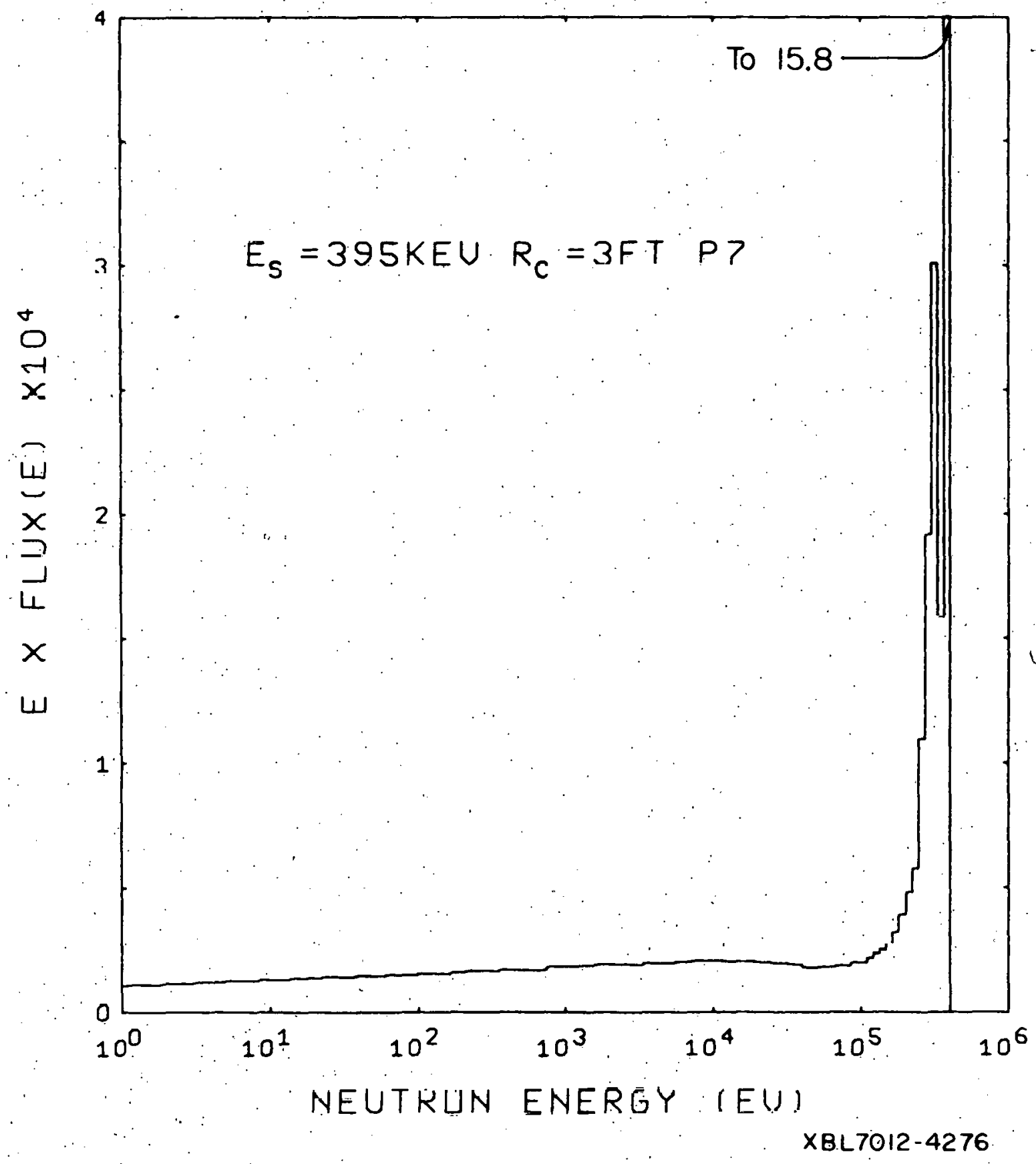

FIGURE 28. Incident Scalar Flux for $E_{S}=395 \mathrm{keV}$ 


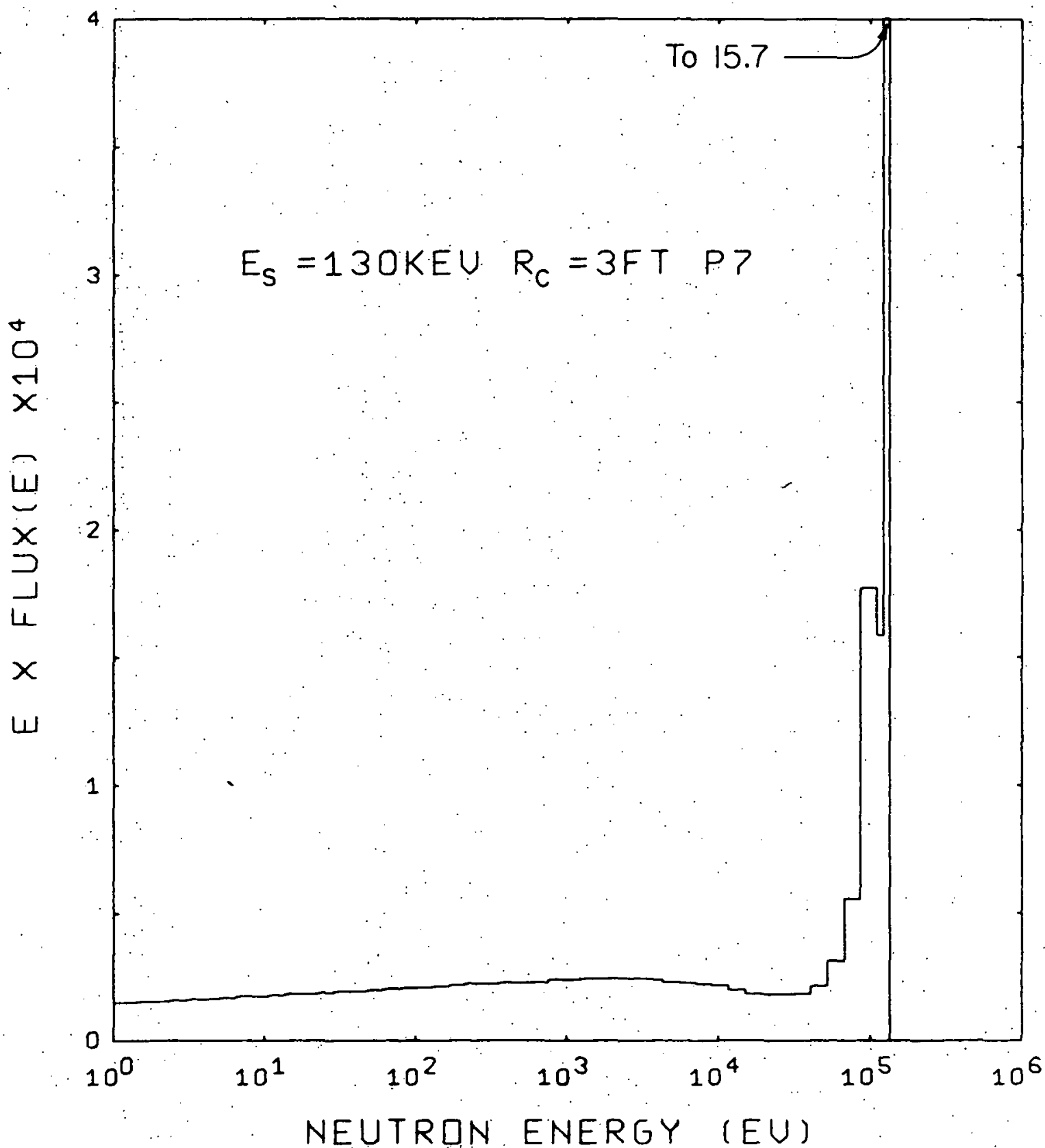

XBL 7012-4277

FIGURE 29. Incident Scalar Flux for $E_{S}=130$ keV 


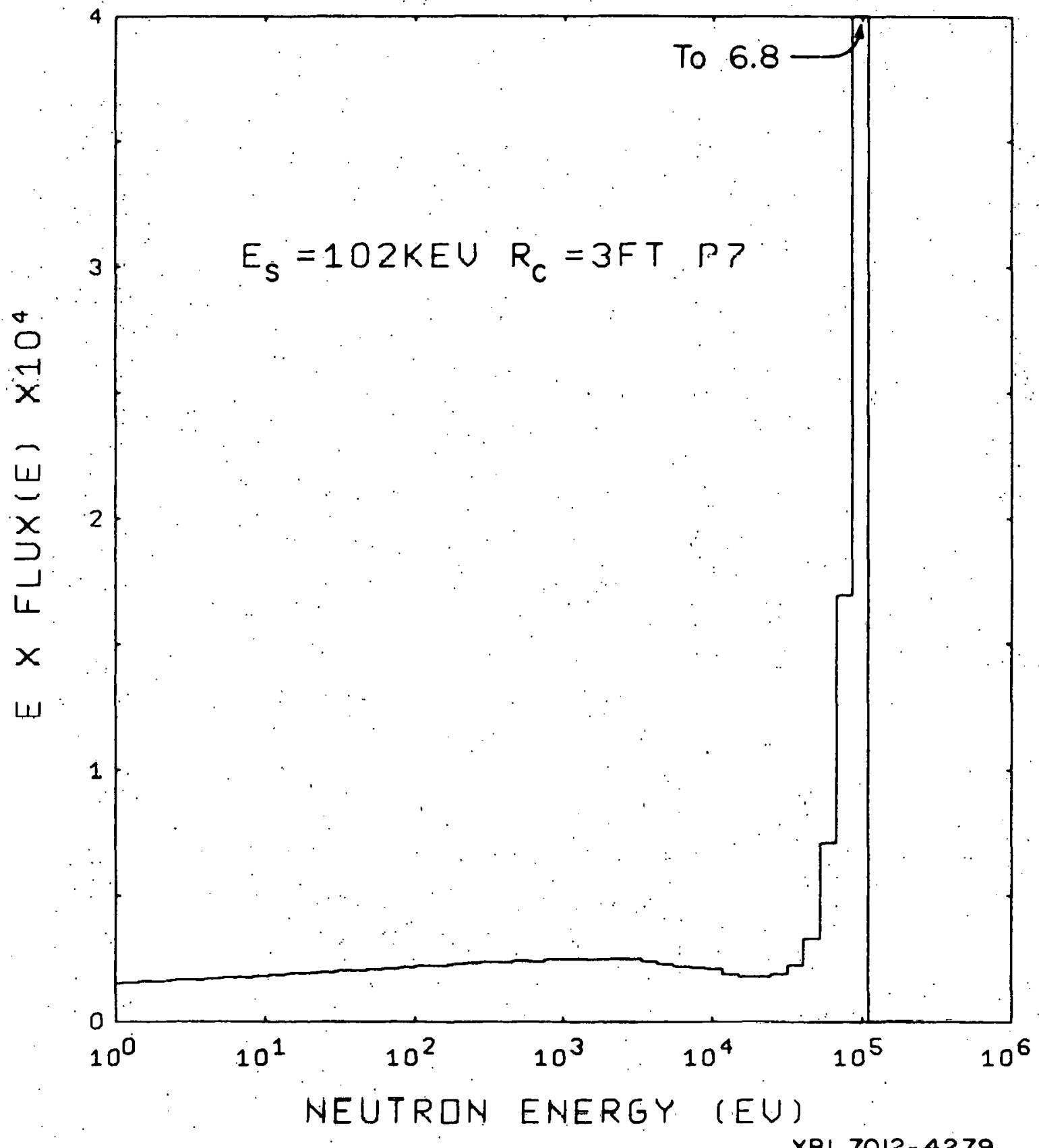

XBL $7012-4279$

FIGURE 30. Incident Scalar Flux for $\mathrm{E}_{\mathrm{S}}=102 \mathrm{keV}$ 


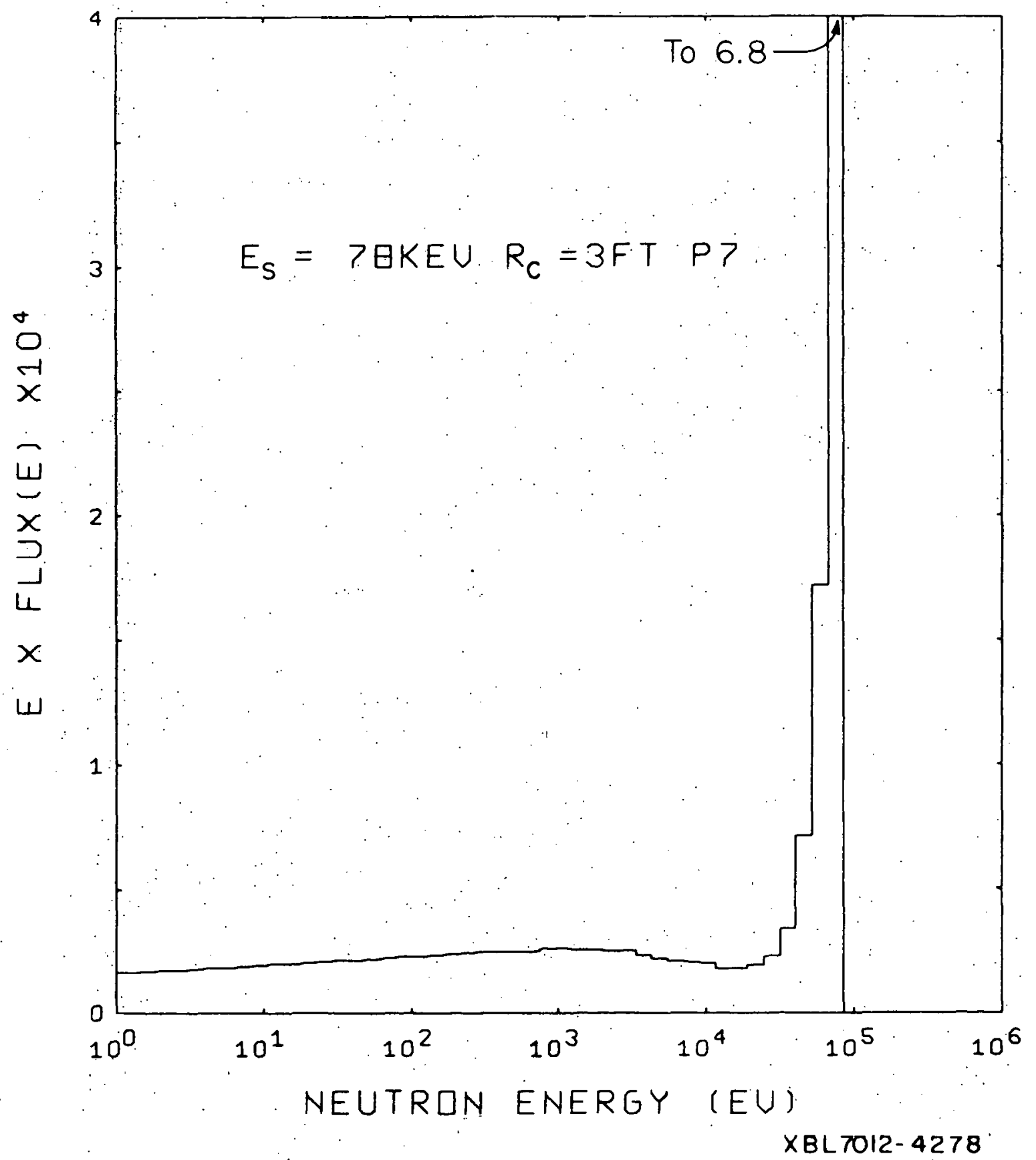

FIGURE 31. Incident Scalar Flux for $E_{S}=78 \cdot \mathrm{keV}$ 
TABLE 7

COMPARISON OF EXPERIMENTAL AND THEORETICAL DATA

\begin{tabular}{|c|c|c|c|c|}
\hline Spectrometer & Source & $\begin{array}{c}A_{\text {sat }}, \mathrm{dps} \\
\text { Expt }\end{array}$ & $\begin{array}{c}\text { B } \\
\text { Asat }_{\text {sat }} \text { dps } \\
\text { Theor. } \\
\text { (norm to I) }\end{array}$ & $\begin{array}{l}\% \text { D1ff. } \\
\frac{B-A}{B} \times 100\end{array}$ \\
\hline \multirow{4}{*}{$\operatorname{In} 4 ; 2,0$} & Mn & 83.2 & 119 & 30 \\
\hline & In & 24.2 & 30.1 & 18 \\
\hline & $\mathrm{Al}$ & 21.9 & 29.8 & 26 \\
\hline & $\mathrm{Sb}$ & 6.3 & $=9.6$ & 34 \\
\hline \multirow[t]{4}{*}{ In $4,4,0$} & Mn & 57.9 & 62.0 & 6.6 \\
\hline & In & 15.0 & 24.2 & 38 \\
\hline & A1 & 12.7 & 17.5 & 27 \\
\hline & $\mathrm{Sb}$ & 4.7 & 6.9 & 32 \\
\hline \multirow{4}{*}{$\begin{array}{c}\text { In } 4,2,1 \\
\because\end{array}$} & Mn & 31.6 & 60.2 & 47 \\
\hline & In & 10.5 & 16.1 & 35 \\
\hline & $\mathrm{A} 1$ & 8.7 & 14.2 & 39 \\
\hline & $\mathrm{sb}$ & 3.6 & 6.8 & 47 \\
\hline \multirow[t]{4}{*}{$\operatorname{In} 4,3,1$} & $\mathrm{Mn}$ & 10.9 & 71.0 & 42 \\
\hline & In & 15.1 & 20.1 & 25 \\
\hline & A1. & 10.1 & 16.1 & 37 \\
\hline & $\mathrm{Sb}$ & 4.0 & 6.7 & 40 \\
\hline
\end{tabular}


are estimated to be 10 to $15 \%$, at maximum. This, plus the inconsistency of certain experimental results (e.g., the failure to measure the iodine cross section correctly at the energy of the $s \dot{b}-B e$ source whose strength was known) Indicate that a more refined calibration experiment should be performed. 


\section{I.V. SUMMARY OF RESULTS AND CONCLUSIONS}

A set of leakage spectrometers (LS's) which make use of activation techniques has been developed. Some of these spectrometers have their peak responses in the keV range. Their resolution, however, generaliy seems too poor to be used for determining rapidiy varying spectra. However, they could determine rough spectra or thc parameters of trial functions describing spectra where there is some information about the shape.

Certain response shapes can be useful for spectal applications. Two examples are: (1) the nearly flat response of In $4,2.5, .625$ between $10^{3}$ and $10^{5}$ eV (see Figure 17) w111 be useful for measuring the total number of neutrons in that range only; (2) determining the total dose, which can be obtalned.by combining LS responses to form the dose-energy relationship, which is normally smooth. lie spectal methods developed here for calculating $\sigma_{L S}(E)$ have proved particable and very accurate. In particular, the use of the adjoint boundary condition to account for the resonance activation of the core material Is expected to be useful in other problems within nuclear $\operatorname{technology}$.

Accurate calculations of the flux spectrum for the photoneutron source have been presented. These show that It was possible to produce nearly monoenergetic isotropic neutrons by the method described. It is felt that more rotingdoxportmonts would agree better with results derived from the theoretical response curves. 
AP P ENDIX A

DEVELOPMENT OF GROUP EQUATIONS USED IN ANISN

The transport equation with spherical symmetry

$$
\begin{aligned}
& \mu \frac{\partial F}{\partial r}(r, u, \mu)+\frac{\left(1-\mu^{2}\right)}{r} \frac{\partial F}{\partial \mu}(r, u, \mu)+\Sigma_{t}(u) F(r, u, \mu) \\
& =\int_{0}^{\infty} d u^{\prime} \int_{4 \pi} d^{\prime} \Omega^{\prime} \Sigma_{S}\left(u^{\prime} \rightarrow u ; \vec{\Omega}^{\prime} \cdot \vec{\Omega}\right) F\left(r, u^{\prime}, \mu^{\prime}\right)+s\left(r, u, \mu^{\prime}\right)
\end{aligned}
$$

will be expanded into spherical harmonics and divided into lethargy groups, the form solved by ANISN.

The scattering kernel $\Sigma_{S}\left(u^{\prime} \rightarrow u, \vec{\Omega}^{\prime} \cdot \vec{\Omega}\right)$, is first approximated by an $L^{\text {th }}$ order Legendre expansion in the cosine of the scattering angle $(\vec{\Omega}, \cdot \vec{\Omega})$

$$
\sum\left(u^{\prime}+u ; \vec{\Omega}^{\prime} \cdot \vec{\Omega}\right)=\frac{2 l+1}{4 \pi} B_{\ell}\left(u^{\prime}+u\right) P_{\ell}\left(\vec{\Omega}^{\prime} \cdot \vec{\Omega}\right)
$$

where $\vec{\Omega}$, and $\vec{\Omega}$ are the initial and final neutron directions respectively.

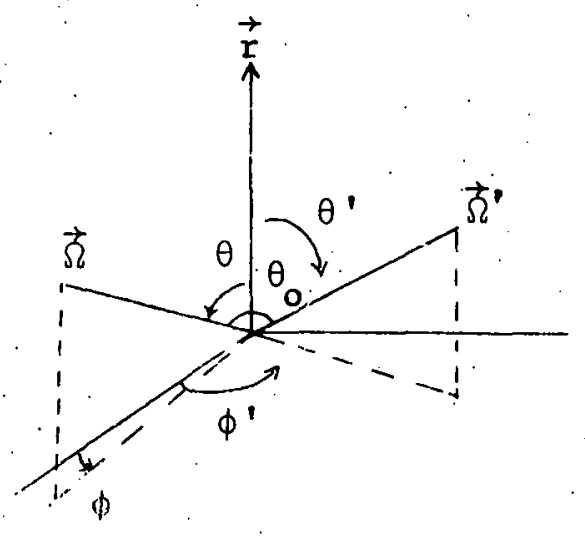

$$
\begin{aligned}
& \theta=\cos ^{-1} \mu \\
& \theta^{\prime}=\cos ^{-1} \mu^{\prime} \\
& \theta_{0}=\cos ^{-1} \mu_{0} \\
& \vec{r} \text { points radially outward }
\end{aligned}
$$


$\vec{\Omega}^{\prime} \cdot \vec{\Omega}$ depends only on $\mu, \mu^{\prime}$ and $\phi^{\prime}-\phi$. The spherical harmonics addition theorem is

$$
\begin{aligned}
& \mathrm{P}_{\ell}{ }^{(\vec{\Omega} \cdot \cdot \vec{\Omega})}=\frac{4 \pi}{2 \ell+1} \sum_{\mathrm{m}=-\ell}^{\ell} \mathrm{Y}_{\ell}^{\mathrm{m}}(\vec{\Omega}) \mathrm{Y}_{\ell}^{\mathrm{m}}\left(\vec{\Omega}^{\prime} \cdot\right) \\
& \therefore \sum_{m=-\ell}^{\ell} \frac{(\ell-\mathrm{m}) !}{(\ell+\mathrm{m}) !} \mathrm{P}_{\ell}^{\mathrm{m}}(\mu) \mathrm{P}_{\ell}^{\mathrm{m}}\left(\mu !^{\prime}\right) \mathrm{e}^{\mathrm{im}\left(\phi^{\prime}-\phi\right)}
\end{aligned}
$$

where $Y_{\ell}^{m}$ are spherical harmonics (* indicating complex conjugation) and $P_{l}^{m}$ are the assorifated Legendreffuctions. Substituting A. 3 into $\dot{A} .2$ and performing the integration over $\phi^{\prime}$ in A. 1

$$
\begin{aligned}
& \int_{0}^{\infty} d u^{\prime} \int_{4 \pi} d \vec{\Omega}^{\prime} \Sigma_{S}\left(u^{\prime}+u ; \vec{\Omega}^{\prime} \cdot \vec{\Omega}\right) F\left(r, u^{\prime}, \mu^{\prime}\right) \\
& =\int_{0}^{\infty} d u^{\prime} \int_{-1}^{1} d \mu^{\prime} \sum_{\ell=0}^{L} \frac{2 \ell+1}{4 \pi} B_{\ell}\left(u^{\prime}+u\right) F\left(x, u, \mu^{\prime}\right) p_{\ell}^{m}(\mu) P_{\ell}^{m}\left(\mu^{\prime}\right) x \\
& \times \int_{0}^{2 \pi} d^{\prime} \sum_{m=-l}^{\ell} \frac{(l-m) !}{(l+m) !} e^{i m\left(\phi^{!}-\phi\right)}
\end{aligned}
$$

but

$$
\int_{0}^{2 \pi} d \phi^{\prime} \sum_{m=-l}^{\ell} \frac{(l-m) !}{(l+m) !} e^{i m\left(\phi^{\prime}-\phi\right)}= \begin{cases}2 \pi & m=0 \\ 0 & m \neq 0\end{cases}
$$


so A. 1 becomes

$$
\begin{aligned}
& \mu \frac{\partial F}{\partial \mu}(r, u, \mu)+\frac{\left(1-\mu^{2}\right)}{r} \frac{\partial F}{\partial \mu}(r, u, \mu)+\sum_{t}(u) F(r, u, \mu) \\
& =\sum_{\ell=0}^{L} \frac{2 \ell+1}{2} P_{\ell}(\mu) \int_{0}^{\infty} d^{\prime} B_{\ell^{\prime}}\left(u^{\prime} \rightarrow u\right) \int_{-1}^{1} d \mu^{\prime} P_{\ell}\left(\mu^{\prime}\right) F\left(r, u^{\prime}, \mu^{\prime}\right) \\
& +S(r, u, \mu)
\end{aligned}
$$

We assume that the 1 ix 1 s constant within the $g^{\text {th }}$ lethargy group

$$
\mathbf{F}(\mathbf{r}, u, \mu)=\mathbf{F}_{\mathbf{g}}(\mathbf{r}, \mu)
$$

$$
u_{g}<u<u_{g+1} \quad g=1, \ldots, G
$$

The flux per solid angle within the $g^{\text {th }}$ group $\mathrm{N}_{\mathrm{g}}$ is then

$$
\mathrm{N}_{g}(r, \mu)=F_{g}(r, \mu) \Delta u_{g}
$$

where

$$
\Delta_{g} \equiv u_{g+1}-u_{g}
$$

Integrating $A .4$ over the $g^{\text {th }}$.group, we obtain

$$
\begin{aligned}
& \mu \frac{\partial}{\partial r} N_{g}(r, \mu)+\frac{1-\mu^{2}}{r} \frac{\partial}{\partial \mu} N_{g}(r, \mu)+\Sigma_{t_{g}} N_{g}(r, \mu) \\
& =\sum_{\ell=0}^{L} \frac{2 \ell+1}{2} P_{\ell}(\mu) \int_{u_{g}}^{u} d u \sum_{g^{\prime}=1}^{u} \int_{u^{\prime}}^{G+1} \int_{g^{\prime}}^{u} d u^{\prime} B_{\ell}\left(u^{\prime}+u\right) \int_{-1}^{1} d \mu^{\prime} P_{\ell}\left(\mu^{\prime}\right) F_{g^{\prime}}\left(r, \mu^{\prime}\right) \\
& +S_{g}(r, \mu),
\end{aligned}
$$


where

$$
\Sigma_{t g} \equiv \int_{u_{g}}^{u} \Sigma_{t}(u) \frac{d u}{\Delta u}
$$

and

$$
\mathrm{s}_{\mathrm{g}}(r, \mu) \equiv \int_{u_{g}}^{\mathrm{u}} \mathrm{g+1}
$$

$$
\begin{aligned}
& \text { Defining } \quad \int_{u}^{u} g+1 \int^{u} g^{!+1} d u^{\prime} B_{\ell}\left(u^{\prime}+u\right) \\
& \mathrm{B}_{\ell}^{\mathrm{g}^{+} \mathrm{g}^{\prime}} \equiv \frac{\mathrm{u}_{\mathrm{g}} \mathrm{u}_{\mathrm{g}^{\prime}}}{\Delta \mathbf{u}_{\mathrm{g}^{\prime}}} \quad \ell=1, \ldots, \mathrm{L}
\end{aligned}
$$

yields for A. 5

$$
\begin{aligned}
& \mu \frac{\partial}{\partial r} N_{g}(r, \mu)+\frac{1-\mu^{2}}{r} \frac{\partial N}{\partial \mu}(r, \mu)+\sum_{t g^{N}}(r, \mu) \\
& =\sum_{\ell=0}^{L} \frac{2 \ell+1}{2} P_{\ell}(\mu) B_{\ell}^{g}+g^{\prime} \int_{-1}^{1} N_{g}\left(r, \mu^{\prime}\right) P_{\ell}\left(\mu^{\prime}\right) d \mu^{\prime}
\end{aligned}
$$




\section{AP PENDIX B}

TESTS OF ANISN

A. Plane Isotropic Source In an Infinite Medium of Hydrogen with Unit Cross Section

(1) P-1 Approximation

With an energy structure of one group of width $10^{-6}$ lethargy units ( $\left.1 . u^{\prime}\right)$ followed by 10 groups of width .011 .4 .9 an S2-P1. ANISN calculation was performed for the above problem. According to the P-1 approximation, the flux at the source lethargy is

$$
\phi(x, 0)=\sqrt{3} \mathrm{e}^{-\sqrt{3} \Sigma_{t} \mathrm{x} \quad \operatorname{Me} 60 \mathrm{a}}
$$

The P-1 flux for neutrons with lethargy slightly greater than the source lethargy was given by Amster Am68 as

$$
\phi\left(x, 0^{+}\right)=3 x e^{-\sqrt{3} \Sigma_{t}}
$$

B. 1 and B.2 are plotted in Figure 32 along with results from the ANISN calculation for the first two groups. It can be seen that except for the iarge mesh spacing ( 1 mean free path); ANISN gives excellent results. The deviations near $x=0$ are caused by the fact that the plane source had to be given finite thickness in ANISN. 


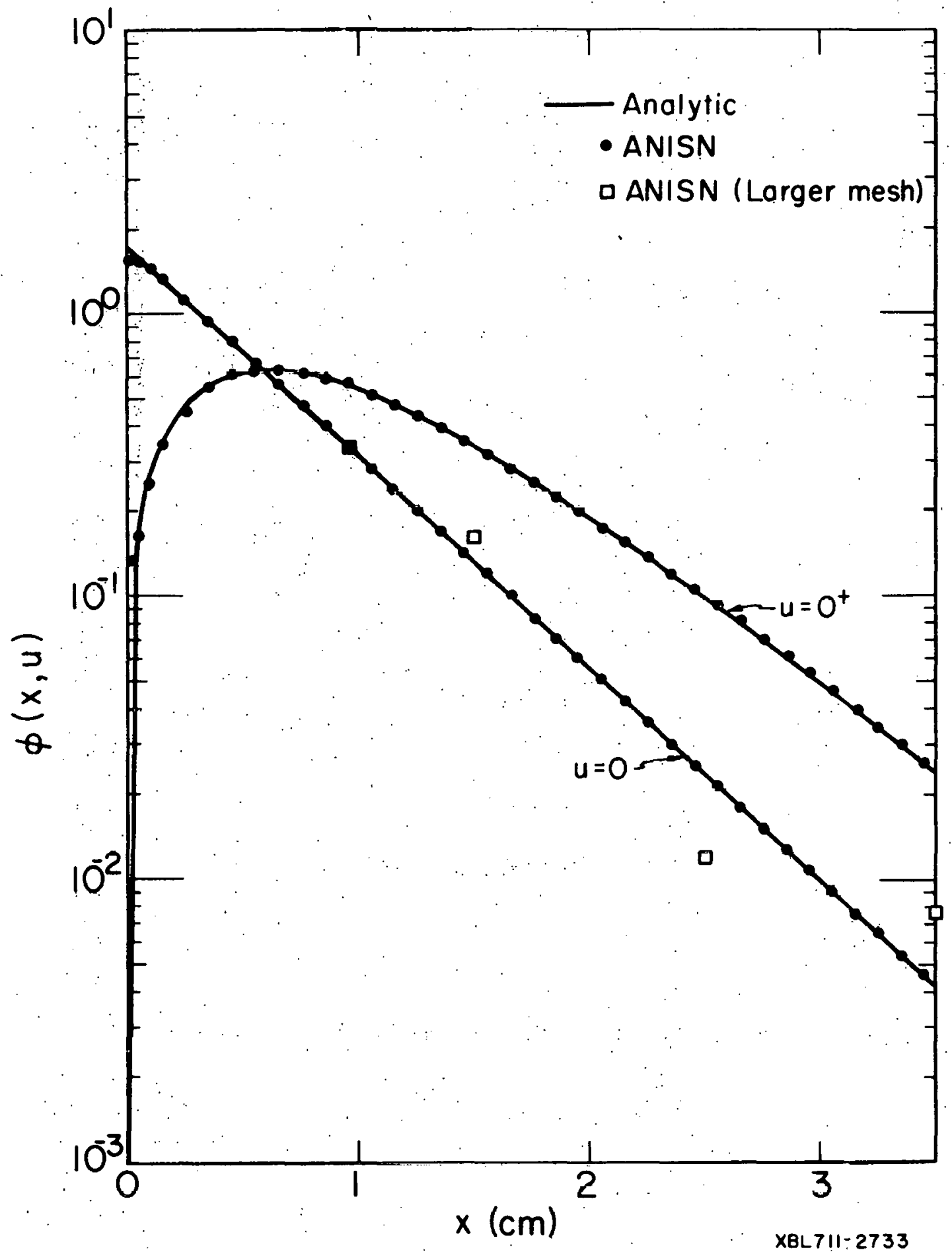

FIGURE 32. P-1 Approximatiul [ur a Plane Source at $x=0$ in añ Infinite Medium. Comparison of analytic solution and ANISN for two lethargles. The squares indlcate ANISN results for a lorger mesh spactng at u=o. 


\section{(2). Spatial Moments}

The spatial moments for the above problem

$$
M_{0}^{n}(u)=\int_{-\infty}^{\infty} x^{n} \phi(x, u) d x
$$

are rigorously given by

$$
\begin{aligned}
& \mathrm{M}_{\mathrm{O}}^{0}=\frac{1}{\Sigma_{\mathrm{S}}}[\delta(\mathrm{u})+1] \\
& \mathrm{M}_{\mathrm{O}}^{2}=\frac{1}{\sum_{\mathrm{S}} 3}\left[\frac{\delta(\mathrm{u})}{3}+\frac{1}{3} \mathrm{e}^{-\mathrm{u} / 2}+\mathrm{u}+\frac{2}{3}\right]
\end{aligned}
$$

A P-1 solution for the above problem gives the exact second moment. Ma47 As a check on the correctness of the ANISN method, the second moment for the last ( $11^{\text {th }}$ ) group (.09-.i 1.u.) was calculated using simpson's rule. The moment determined in this manner was only . $5 \%$ different from the rigorous value determined by $B .3$, indicating that for this problem, the ANISN lethargy dependence is accurate.

\section{B. Pure Absorber Spherical Geometry}

The flux due to an isotroplc spherical shell source emitting 1 neutron/sec in a purely absorbing medium is given $\operatorname{CaS}^{3}$ by

$$
\phi(r)=\frac{1}{8 \pi r a}\left\{\frac{E_{1}\left[\Sigma_{a}(a-r)\right]-E_{1}\left[\Sigma_{a}(a+r)\right]}{2}\right\}
$$


where a is the radius of the source and $E_{n}$ is the $n^{\text {th }}$ order exponential integral function. Figure 33 shows B. 4 and the results of a $\mathbf{8} 8$ ANISN calculation plotted for $a=2.4$ and $\sum_{a}=1$.

\section{Energy Dependent, Heavy Scatterer}

The spatially independent collision density $F(u)=$ $\Sigma_{S}(u) \phi(u)$ due to a delta-funcrion source $\delta(u)$, is given ${ }^{M e 60 b}$ by

$$
\begin{gathered}
F(u)=\frac{e^{u v / 1-\alpha}}{1-\alpha} ; 0<u<\ln (1 / \alpha) \\
F(u)=\frac{1-\alpha^{1 / 1-\alpha}}{1-\alpha} e^{\alpha u / 1-\alpha}-\frac{\alpha}{(1-\alpha)^{2}}\left(u-\ln \frac{1}{\alpha}\right) e^{\alpha u / 1-\alpha} ; \\
\ln \frac{1}{\alpha}<u<2 \ln \frac{1}{\alpha}
\end{gathered}
$$

for the first two collision intervals, where

$$
\alpha=\left(\frac{A-1}{A+1}\right)^{2}
$$

A 20 group ANISN solution and B.5 are plotted in Figure 34 for $A=63.5$. 


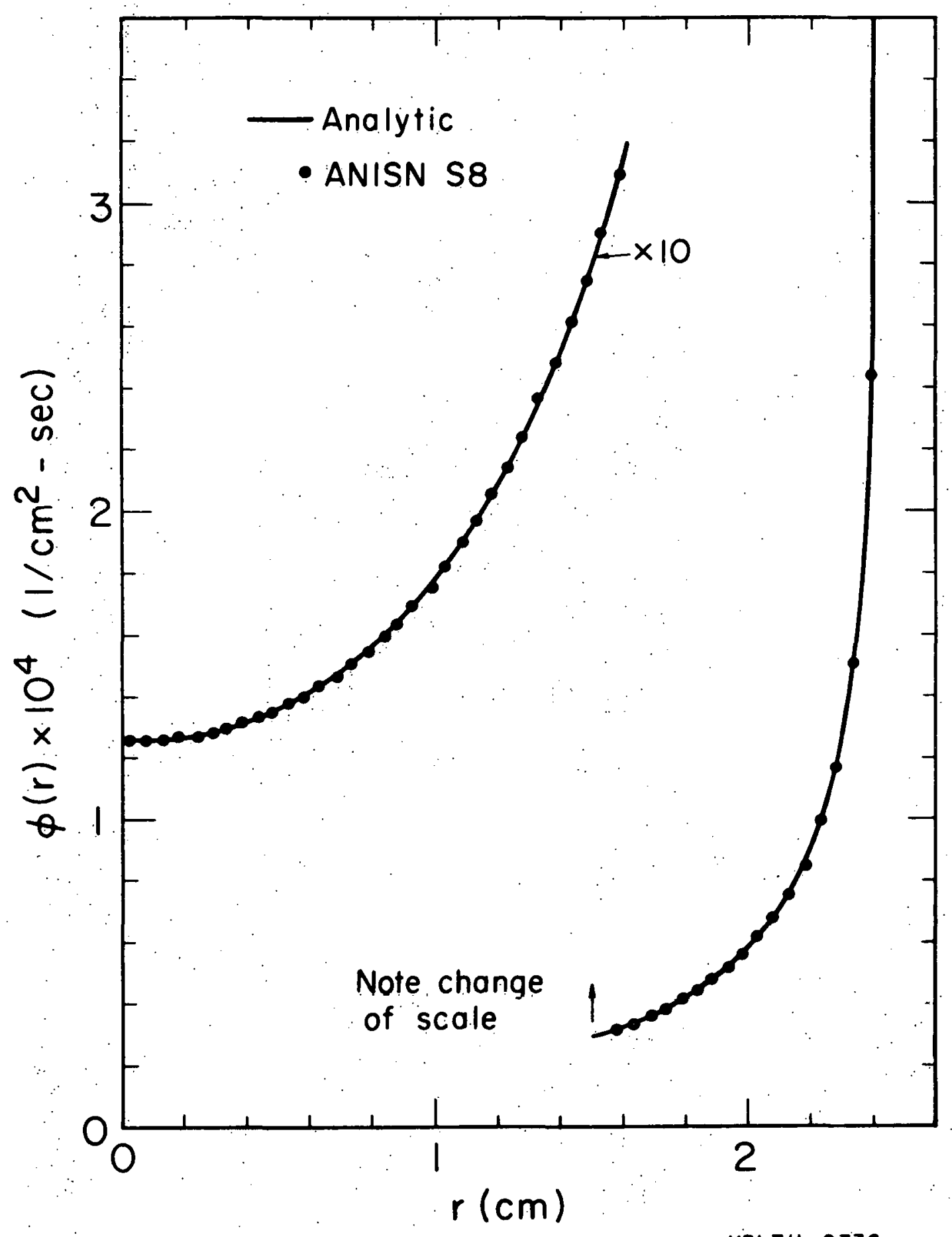

XBI $111-2736$

FIGURE 33. Flux in a Purely Absorbing. Medium: Due to an Isotropic Shell Source at $r=2.4 \mathrm{cr}$ : 


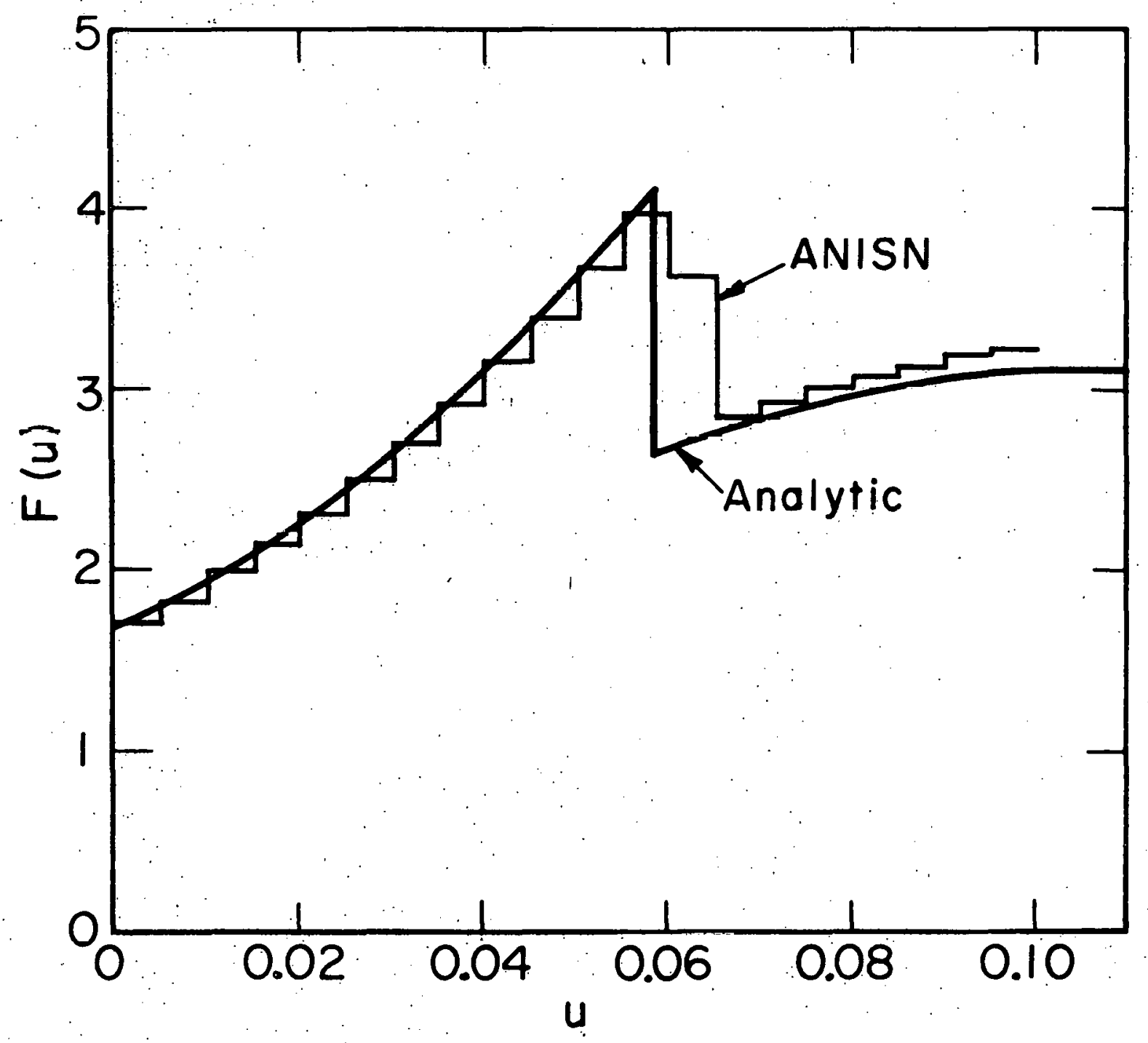

$\times B L 711-2737$

FIGURE 34. Collision Density over. Two Scattering Intervals for a Non-Absorbing Infinite Medium with $A=63.5$ and a Delta-Punction Source at $u=0$ 
APPENDIX C

CROSS SECTIONS AND ENERGY TRANSFER ARRAY

\section{A. Resonance Cross Sections}

The resonance cross sections required for the adjoint boundary condition are generated using a single-level Brelt-Wigner formula:

The radiative capture cross section is

$$
\sigma_{a}(\dot{E})=2.60 \times 10^{6} \sum_{1} g_{i} \frac{\Gamma_{n i}^{0} \Gamma_{i}}{\Gamma_{i}^{2} \sqrt{E}} \frac{(1+1 / A)^{2}}{\left(\frac{E-E_{i}}{\Gamma_{i} / 2}\right)^{2}+1}
$$

where the total width has been taken to be

$$
\Gamma_{i}=\Gamma_{n i}^{0} \sqrt{E_{1}}+\Gamma_{\gamma 1}
$$

$\Gamma_{n}^{0}$ is the reduced neutron width $\left(\Gamma_{n} / \sqrt{E_{0}}\right)$ and $\Gamma_{\gamma}$ is the gamma ray width. E is the neutron energy, $E_{i}$ is the energy of the $i^{\text {th }}$ resonance, and $A$ is the mass of the target nucleus. The statistical spin factor is

$$
g=\frac{1}{2} \frac{2 J+1}{2 I+1}
$$

where $I$ is the target spin and $J$ is the spin of the compound nucleus.

In a simflar manner, the resonance scattering cross daction to glven by 


$$
\begin{aligned}
& \sigma_{S}(E)=\sigma_{F}+\sum_{i}\left[2.6 \times 10^{6} g_{i} \frac{\left(\Gamma_{n i}^{0}\right)^{2}}{\Gamma^{2}} \frac{(1+1 / A)^{2}}{\left(\frac{E-E_{i}}{\Gamma / 2}\right)^{2}+1}\right. \\
& \left.+1.61 \times 10^{3} g_{i} \frac{\Gamma_{n i}^{0} \sqrt{\sigma_{P}}}{\Gamma_{i}} \frac{\frac{E-E_{i}}{\Gamma / 2}(1+1 / A)^{2}}{\left(\frac{E-E_{i}}{\Gamma / 2}\right)^{2}+1}\right]
\end{aligned}
$$

where $\sigma_{p}$ is the spin independent potential scattering oroso section. The second term in the summation accounts for the interference between resonance and potential scattering. Me60C,Hu66

The total cross section is

$$
\sigma_{t}(E)=\sigma_{a}(E)+\sigma_{S}(\dot{E})
$$

\section{B. Average Unresolyed Resonance Cross Section}

The average capture cross section in the unresolved resonance region for neutrons of orbital angular momentum, ' $l$, assuming a Porter-Thomas probability distribution of reduced neutron widths

$$
P(x):=\frac{1}{\sqrt{2 \pi} x} \cdot e^{-x / 2}
$$

with

$$
x=\Gamma_{n \ell}^{o} / \check{\Gamma}_{n \ell}^{\circ}
$$


is given by Garrison and Roos Ga61 as

$$
\bar{\sigma}_{a}^{\ell}(E)=\frac{4.09 \times 10^{9}}{\sqrt{E}} \frac{v_{\ell}(E) \bar{\Gamma}_{n \ell}^{\circ}}{D} \phi\left(\theta_{\ell}\right)(2 \ell+1)(1+1 / A)^{2}
$$

where $\bar{\Gamma}_{\text {no }}^{l}$ is the average reduced width obtained from resolved resonance data. The neutron penetrability functions, $v_{\ell}(E)$ are given by

$$
\begin{aligned}
v_{0} & =1 \\
v_{1} & =Y^{2} /\left(1+Y^{2}\right) \\
v_{2} & =Y^{4} /\left(9+3 Y^{2}+Y^{4}\right) \\
V_{3} & =Y^{6} /\left(225+45 Y^{2}+6 Y^{4}+Y^{6}\right)
\end{aligned}
$$

and

$$
V_{4}=Y^{8} /\left(1025+1575 Y^{2}+135 Y^{4}+10 Y^{6}+Y^{8}\right)
$$

where

$$
Y=\frac{A^{1 / 3} E^{1 / 2}}{3250}
$$

$\bar{\Gamma}_{n \ell}^{\circ} / D$ is the neutron strength function and

$$
\theta_{\ell}=\frac{\Gamma_{\gamma}^{/ D_{\ell}}}{\left(\begin{array}{c}
\Gamma_{\ell} \\
0
\end{array}\right)^{\prime \prime} v_{\ell}} \times 10^{-3}
$$


The level. spacing per. spin state $D_{\ell}$ is obtained from observed level spacing, $D_{0}$; by

$$
D_{\ell}=D_{0} \frac{2 \ell+1}{\sum_{J} g_{J}^{2}}
$$

The sum $\sum_{\mathrm{J}} g_{\mathrm{J}}^{2}$ extends over the statistical spin factors for allallowable combinations of the spin $J$ of the compound nucleus for a target spin $I$ and an orbital angular momentum $l$ of the neutron. The function $\phi\left(\theta_{\ell}\right)$ is given by

$$
\phi\left(\theta_{\ell}\right)=\int_{0}^{\infty} \frac{x P(x)}{1+x / \theta_{\ell}} d x
$$

$\sigma_{l}^{\ell}(E)$ is a smooth function of energy, so the average capture cross section for group $g$ is given by

$$
\left(\bar{\sigma}_{a}\right) g=\sum_{l=0}^{\ell} \frac{\max _{\bar{\sigma}} \ell\left(E_{g}\right)+\bar{\sigma}_{\gamma}^{\ell}\left(E_{g+1}\right)}{2} \because
$$

Unresolved resonance scattering is usually either neglected $\left(\left(\bar{\sigma}_{g}\right)^{g}\right)=\sigma_{P}$, (potential scattering) or determined In a manner similar to $\left(\bar{\sigma}_{a}\right)^{g}$, neglecting interference effects.

\section{Transfer Array}

The energy transfer array or scattering kernel expanstion coefficients $\mathrm{B}^{g+g^{\prime}}$ (see Eq. A.6) written in terms of an integral over energy are given by 


$$
B_{\ell}^{g^{+} g^{\prime}}=\frac{1}{\Delta u_{g}} \int_{E}^{E} d \int_{E^{\prime}}^{E+1} \int_{g^{\prime}}^{E} \frac{d E^{\prime}}{E^{\prime}} B_{\ell}\left(E^{\prime} \rightarrow E\right)
$$

The factor 1/E' corresponds with the change of variables to a weighting flux that is constant in lechargy. $B_{l}^{g+g}$ is composed of three terms

$$
\left(B_{l}^{g+g^{\prime}}\right)_{n}+\left(B_{l}^{g+g^{\prime}}\right)_{n}+\left(B_{l}^{g+g^{\prime}}\right)_{2 n}
$$

denoting elastic scattering, Inelastic scattering and an $(n, 2 n)$ reaction respectively. In each case the transfer coefficient $B_{\ell}\left(E^{\prime} \rightarrow E\right)$ is obtained from a differential cross section

$$
B_{\ell}\left(E^{\prime}+E\right)=\int_{-1}^{1} d \mu_{0} \sigma\left(E^{\prime}+E, \mu_{0}\right)
$$

where $\mu_{0}$ is the cosine of the scattering angle in the $1 \mathrm{ab} \cdot \mathrm{sys}$ tem.

For elastic scattering

$$
\begin{aligned}
& \sigma_{n}\left(E^{\prime}+E, \mu_{0}\right)=\sigma_{n}\left(E^{\prime}\right) \sum_{m} \frac{2 m+1}{2} f_{m}(E) P_{m}\left(\mu_{c}\right) \frac{\partial \mu_{c}}{\partial \mu_{0}} \times \\
& \times \delta\left[E-E^{\prime}+\frac{1-\alpha}{2} E^{\prime}\left(1-\mu_{c}\right)\right]
\end{aligned}
$$

where $\sigma_{n}\left(E^{\prime}\right)$ is the elastic scattering cross section, $\mu_{c}$ Is the cosine of the scattering angle in the center- 
of-mass (CM) system, $f_{m}\left(E^{\prime}\right)$ are determined from the $C M$ differential cross section $\sigma_{n}\left(E^{\prime}, \mu_{c}\right)$ and

$$
\alpha=\left(\frac{A-1}{A+1}\right)^{2}
$$

The delta-function arises because the final energy $E$ is determined by kinematics for a given initial energy $E^{\prime}$ and $\mu_{c}$. Changing the integration variable in $C .5$ to energy and noting that

$$
\frac{d \mu_{c}}{d \mu_{0}} d \mu_{0}=d \mu_{c}=\frac{2}{(1-\alpha) E} \cdot d E
$$

and using C.6 yields:

$$
\begin{gathered}
\left(B_{\ell}\left(E^{\prime}+E\right)\right)_{n}=\frac{\sigma_{n}\left(E^{\prime}\right)}{(1-\alpha) E^{\prime}} \cdot P_{\ell}\left(\mu_{0}\right) \sum_{m}(2 m+1) f_{m}\left(E^{\prime}\right) P_{m}\left(\mu_{c}\right) \\
\text { for } \alpha E^{\prime} \leqq E \leqq E^{\prime}
\end{gathered}
$$

otherwise

$$
\left(B_{\ell}\left(E^{\prime}+E\right)\right)_{n}=0
$$

For lnelastic scattering for which the discrete level excitation cross sections $\sigma_{n^{\prime} k}\left(E^{\prime}\right)$ and $Q$-value are known 


$$
\begin{aligned}
& \sigma_{n^{\prime} k}\left(E^{\prime}+E, \mu_{0}\right)=\sigma_{n^{\prime} k}\left(E, \mu_{0}\right) \delta\left[E-E^{\prime}+\frac{A W_{k}}{A+1}+\right. \\
& \left.+\frac{2 A E^{\prime}}{(A+I)^{2}}\left(1-\mu_{c}\right) \sqrt{1-\frac{(A-1)}{A E^{\prime}} W_{k}}\right]
\end{aligned}
$$

where $W_{k}=-Q$. Following the same steps as for elastic scatterịg above

$$
\begin{aligned}
& \left(B_{\ell k}\left(E^{\prime}+E\right)\right)_{n}=\frac{\sigma_{n^{\prime} k}\left(E^{\prime}\right) P_{\ell}\left(\mu_{o}\right)}{(1-\alpha) E^{\prime} \sqrt{1-\frac{(A+1)}{A E^{\prime}} W_{k}}} \times \\
& \times \sum_{m}(2 m+1) \dot{f}_{m, k}\left(E^{\prime}\right) P_{\ell}\left(\mu_{c}\right)
\end{aligned}
$$

for

$$
\left(\frac{1+A \sqrt{1-\frac{(A+1)}{A E^{\prime}}} W_{k}}{1+A}\right)^{2} \geqq \frac{E}{E}, \geqq\left(\frac{1-A \sqrt{1-\frac{(A+1)}{A E^{1}} W_{k}}}{1+A}\right)^{2}
$$

otherwise

$$
\left(B_{\ell k}\left(E^{\prime} \rightarrow E\right)\right)_{n} i=0
$$

For $(n, 2 n)$ reactions and that portion of the inelastic cross section that cannot be treated as individual leve1s a statistical model is used. An evaporation type spectrum is assumed

$$
N\left(E^{\prime}, E\right)=C\left(E^{\prime}\right) E e^{-E / \theta\left(E !^{\prime}\right)}
$$


where $C\left(E^{\prime}\right)$ is the normalization factor and $\theta\left(E^{\prime}\right)$ is the nuclear temperature

$$
\theta\left(E^{\prime}\right)=k T \simeq 3226 \sqrt{E^{\prime} / A}
$$

The differential inelastic scattering kernel in the laboratory system is

$$
\sigma_{1 \mathrm{n}}\left(E^{\prime}+E, \mu_{0}\right)=\frac{\sigma\left(E^{\prime}\right)}{2} \frac{d \mu_{c}}{d \mu_{0}} N\left(E^{\prime}, E\right)
$$

where it is assumed that the angular distribution of secondary neutrons is isotropic. The subscript in stands either for $n^{\prime}$ or $2 n$. Thus

$$
\left(B_{0}\left(E^{\prime}, E\right)\right)_{\text {in }}=\sigma_{\text {in }}\left(E^{\prime}\right) N\left(E^{\prime}, E\right) \quad E<E^{\prime}
$$

Of these three contributiong, the elaotic trangfer is the most important, the other two being non-zero on $y$ above $100 \mathrm{keV}$, for the isotopes of interest here. 


\section{REFERENCES}

Ad67 J. Adir and K.D. Lathrop, "Theory of Methods Used in the GGC-3 Multigroup Cross Section Code", USAEC Report GA-7156 (1967).

Al65 E. Albold and P. von Blankenhagen, "Elne Messung des isomeren Aufspaltverhältmisse bel Aktivierung in den ersten 3 Indium-Resonanzen", Nukleonik, $\underline{7(7)}$, 406 (1965).

Am68. H.J. Amster, "An Analysis of Methods for Calculating Spatially Dependent Neutron Slowing Down Distributions", Nuc: Sc1. Eng:, 34, 313 (1968).

Be63 K.H. Beckurts, M. Brose, M. Kroche, G. Krüger, W. Pönitz and H. Schmidt, "Thermal Activation Cross Sections and Resonance Integrals of Indium", Nuc. Sc1. Eng., 17, 329,(1963).

Bi70 S.E. Binney, "Calibration of Boron-Covered Multisphere Detectors Using Resonance Activation for Neution Spectrometery in the keV Region", Ph.D. Thesis, U. of Calffornia, Berkeley (1970).

Bo60 T.W. Bonner, "Measurements of Neutron Spectra from F1ssion", Nuc. Phys., 23, 116 (1961).

Br60 R.L. Bramblett, R.I. Ewing and T.W. Bonner, "A New Type of Neutron Spectrometer", Nuc. Inst. Meth., 9, $1(19.60)$.

Ca53 K.M. Case, F. de Hoffman and G. Placzek, Introduction to the Theory of Neutron Diffusion, Vol. 1 (Los Alamos Scientific Laboratory, 1953), p. 16 .

Di65 G. DiCola and A. Rota, "Calculation of Differential Fast Neutron Spectra from Threshold-foil Activation Data by Least-Squares Series Expansion Methods", Nuc. Sc1. Eng., 23, 344 (1965).

Do60 F. Domonic and V.L. Sailor, "Dependence of the In"16 Activation Ratio on Neutron Energy", Phys. Rev., $119(1), 208$ (.1960).

Dr58 J.E. Draper, C.A. Fenstermacher and H.L. Schulz, "Variations in Spectra of Resonance Neutron Capture Gamma Rays In Indium;" Phys. Rev.;111(3), 906 (1958).

Eng 7 W. Engle, "A Users Manual for ANISN, A One Dimenstonal Diacreto Ordinates Transport Code with Anisotropic Scattering," USAEC Report K-1693 (1967). 
Ga61 J.D. Garrison and B.W. Roos, "Fission Product Capture Cross Sections", USAEC Report GA-2112 (1961):

Go64 R. Gold, "An Iterat1ve Unfolding Method for Response Matrices", USAEC Report ANL-6984 (1964).

Go66 M.D: Goldberg, S.F. Mughabghab; S.N. Burohit; B.A. Magurno, and V.M. May, "Neutron Cross Sections", USAEC Report BNL-325 Second. Edition Supplement No. 2 (1966).

Ho66 H.C. Honeck, "ENDF/B Specifications for an Evaluated Nuclear Data Pile for Reactor Applications"., USAEC Report BNL-50066 (T-467) ENDF 102 (1966).

K160 C.M. Kim, "A Theoretical Comparison of $4 \pi$ FastNeutron Spectrometers", (M.S. Thes1s) USAEC Report UCRL-9504 (1960).

La65 K.D. Lathrop, "DTF-IV, a Fortran IV Program for Solving the Multigroup Transport Equation with Anisotropic Scattering", USAEC Report LA-3373 (1965).

Ma47 R.E. Marshak, "Theory of the Slowing Down of Neutrons by Elastic Coll1sions with Atomic. Nuclei", Rev. Mod: Phys., 19, 185 (1947).

Mc67 W.N. McE1roy, S. Berg, and G. Gigus, "Neutron Spectral Determination by Foll Activation", Nic. Sci. Eng., 27, 533 (1967):

Mc69 W.N. McElroy, S. Berg, J.B. Crockett and R.J. Tuttle, "Measurement of Neutron. Flux Spectra by a Multiple Foll with Reactor Physics Calculations and Spectrometer Measurements", Nuc. Sci. Eng,, 36, 15 (1969).

Me 60 R.V. Meghreblian and D.K. Holmes, Reactor Analysio, McGraw-Hi11; New York (1960). (a, p.182; b, p.94; c, p.673)

Me64 S.K. Metha, "Mndiffeation of Bonner Sphere Spectrometer to Measure Spectra of Low-Energy. Neutrons from $(\alpha, n)$ Sources", USAEC Report ORNL-3714, p. 80 (1964).

Me65 S.K. Metha, "A New Method for the Evaluation of Neutron Emission, Kerma, Kerma Equivalent, Dose Equivalent of the spectrum Below 1 MeV for $(\alpha, n)$ Sources, Us Ing Bonner Spheres", USAEC Report ORNL-TM-1171. (1965). 
Po65 W. Pönitz, "The Spin of the 2-sec Isomeric State of In $16 \%$, Nuc. Phys ,66, 297 (1965):

Ro69 Y.T. Routt1, "H. Igh Energy Neutron Spectroscopy with Activation Detectors, Incorporating New Methods for the Analysis of Ge(Li) Gamma Ray Spectra and the Solution of Fredholm Integral Equations", Ph.D. Thesis, USAEC Report UCRL-18514 (1969).

Se59 D.S. Selengut, "Variational Analysis of MultiDimensional Systems", USAEC Report HW-59126, p. 89 (1959).

St68 A. Staub, D. Harris and M. Goldsmith, "Analysis of a Set of Critical Homogeneous U-H2. Spheres", Nuc. Sci. Eng., 34, 263 (1968).

Th53 B.W. Thompson, "Fast-Neutron Scintillation Survey Meter", USAEC Report UCRL-2357 (1953).

Tr68 D.K. Trubey and B.F. Maskewitz, Ed., "A Review of the Discrete ordinates $S_{n}$ Method for Radiation Transport Calculations", USAEC Report ORNL-RSIC-19 $(1968)$. 


\section{ACRNOWLEDGMENTS}

I would like to express. my gratitude. to the three men with whom I worked on th1s project. Dr. Hans Mark and Dr: Stephen Binney, who formulated the project, helped me in innumerable ways to understand the experimental work and guided my analysis of that work. Special thanks go to Dr. Harvey Amster, my thesis advisor, who helped in the development of the methods used here, and who was alwayg willing to give advice for their implementation.

I would also like to thank Dr. Roger Wallace and Dr. Kenneth watson, who read my thesis and provided valuable comments.

Finally, I would like to thank my wife Susan, whose encouragement and companionship contributed to the completion of this work.

I recelved tinancial support from an AEC Special Fellowship in Nuclear science and Engineering, and a Research Asoistantship in the Health Physics Department of the Lawrence Radiation Laboratory at Berkeley. Th1s work was partially supported by AEC Contract AT 0911-10 34 Proj. 161. The bulk of the work was supported by the Health Physics Department of LRL-Berkeley under the auspices of the Atomic Energy Commission. 


\section{LEGAL NOTICE}

This report was prepared as an account of work sponsored by the United States Government. Neither the United States nor the United States Atomic Energy Commission, nor any of their employees, nor any of their contractors, subcontractors, or their employees, makes any warranty, express or implied, or assumes any legal liability or responsibility for the accuracy, completeness or usefulness of any information, apparatus, product or process disclosed, or represents that its use would not infringe privately owned rights. 
